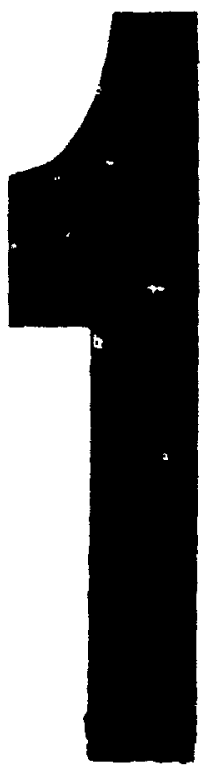

PM-1 3\%" $x 4 "$ PHOTOCRAPHIC MICROCOPY TARGET NBS t010a ANSI/ISO \#2 EQUIVALENT

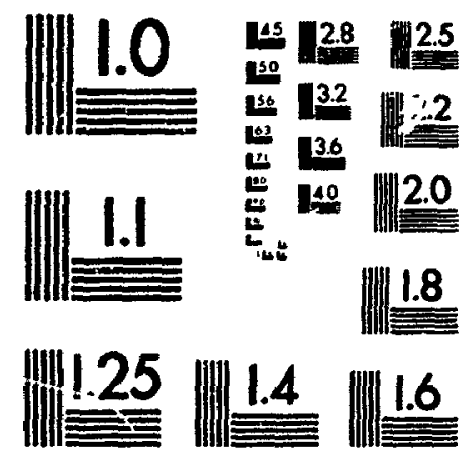

PRECISIONSM RESOLUTIOH TARGETS 
National Library

of Canada

Acquisitions and

Bibliographic Sevices Branch

395 Weillington Street

Ottawa, Ontario

KIA ON4
Bibliotheque nationale

du Canada

Direction des acquisitions et

des services bibliographiques

395, ne Wellington

Ottawa (Ontario)

KIAONA
Youn the volio retorence

Our the Nolre reference

NOTICE

AVIS

The quality of this microform is heavily dependent upon the quality of the original thesis submitted for microfilming. Every effort has been made to ensure the highest quality of reproduction possible.

If pages are missing, contact the university which granted the degree.

Some pages may have indistinct print especially if the original pages were typed with a poor typewriter ribbon or if the university sent us an inferior photocopy.
La qualité de cette microforme dépend grandement de la qualité de la thèse soumise au microfilmage. Nous avons tout fait pour assurer une qualité si poérieure de reproduction.

S'il manque des pages, veuillez communiquer avec l'université qui a conféré le grade.

La qualité d'impression de certaines pages peut laisser à désirer, surtout si les pages originales ont été dactylographiées à l'aide d'an ruban usé ou si l'université nous a fait parvenir une photocopie de qualité inférieure.

La reproduction, même partielle, de cette microforme est soumise à la Lol canadienne sur le droit d'auteur, SRC 1970, c. C-30, et ses amendements subséquents.
Reproduction in full or in part of this microform is governed by the Canadian Copyright Act,

R.S.C. 1970, c. C-30, and subsequent amendments. 


\title{
SERIES SOLUTIONS OF THE \\ LORENZ SYSTEM AND THE DUFFING EQUATION
}

\author{
by
}

Andrea Zypchen, B.Sc.

A thesis submitted to

the Faculty of Graduate Studies and Research

in partial fulfillment of the :equirements for the degree of

Master of Science

Department of Mathematics and Statistics

Carleton University

Ottawa. Canada

April. 1994

(c) Copyright, 1994

Andrea Zypchen 
National Library

of Canada

Acquisitions and

Bibliographic Senvices Branch

395 Wellingion Street

Ottawa, Ontarin

KiA ONA
Bibliotheque nationale

du Canada

Direction des acquisitions et

des services bibliographiques

395. rue Wellington

Ottawa (Ontario)

KIA ONA
Your bite Votrenelerence

Our the Notienderence
THE AUTHOR HAS GRANTED AN IRREVOCABLE NON-EXCLUSIVE LICENCE ALLOWING THE NATIONAL LIBRARY OF CANADA TO REPRODUCE, LOAN, DISTRIBUTE OR SELL COPIES OF HIS/HER THIESIS BY ANY MEANS AND IN ANY FORM OR FORNIAT, MAKING THIS 'THESIS

AVAILABLE TO INTERESTED PERSONS.
L'AUTEUR A ACCORDE UNE LICENCE IRREVOCABLE ET NON EXCLUSIVE PERMETTANT A LA BIBLIOTHEQUE NATIONALE DU CANADA DE REPRODUIRE, PRETER, DISTRIBUER OU VENDRE DES COPIES DE SA THESE DE QUELQUE MANIERE ET SOUS QUELQUE FORME QUE CE SOIT POUR METTRE DES EXEMPLAIRES DE CETTE THESE A LA DISPOSITION DES PERSONNE INTERESSEES.
THE AUTHOR RETAINS OWNERSHIP OF THE COPYRIGHT IN HIS/HER THESIS. NEITHER THE THESIS NOR SUBSTANTIAL EXTRACTS FROM IT MAY BE PRINTED OR OTHERWISE REPRODUCED WITHOUT HIS/HER PERMISSION.
L'AUTEUR CONSERVE LA PROPRIETE DU DROIT D'AUTEUR QUI PROTEGE SA THESE. NI LA THESE NI DES EXTRAITS SUBSTANTIELS DE CELLECI NE DOIVENT ETRE IMPRIMES OU AUTREMENT REPRODUITS SANS SON AUTORISATION.

ISBN $\quad 0-315-98640-9$ 


\section{Series Solutions of the Lorenz System and the}

Name Duffing Equation

Dissertation Abstrocts International is arranged by troad, general subject categories. Please select the one subject which most nearly describes the content of your dissertation. Enter the corresponding four-digit code in the spaces provided.

\section{Mathematics}

01465 U.M.I

SUBNeT CoDE

\section{Subject Congories}

\section{TH HUMANITITS AND SOCIAL SCIINCES}
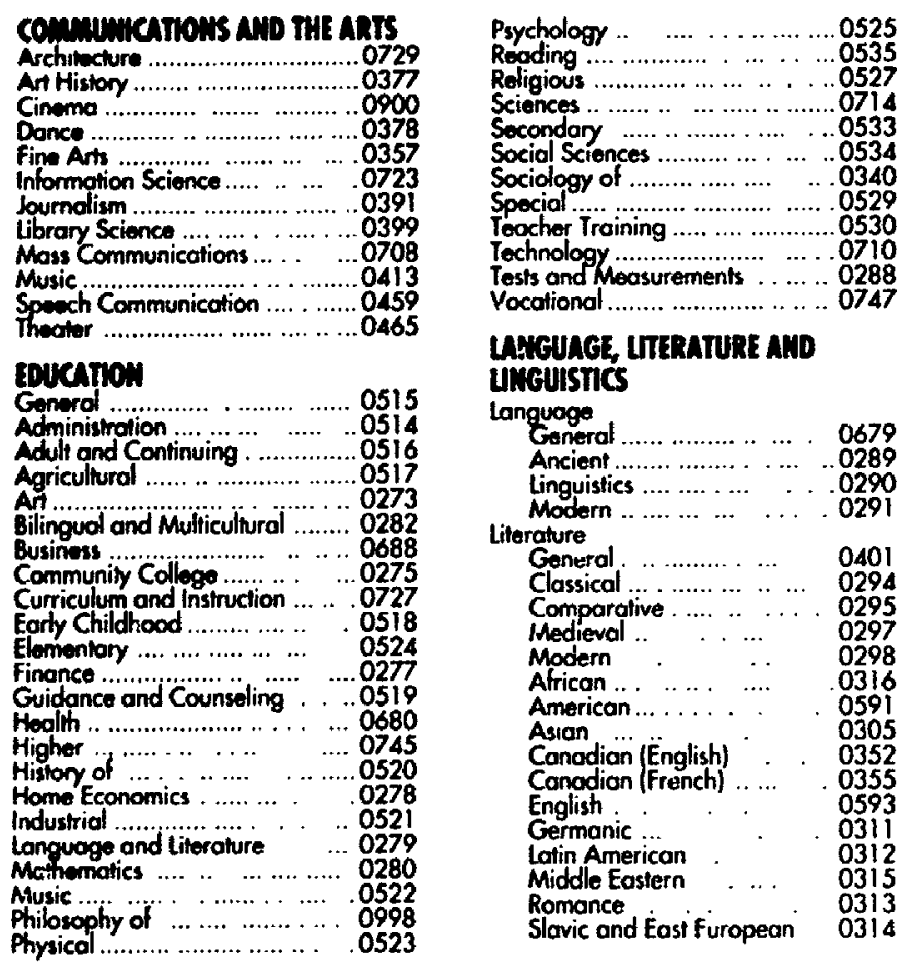

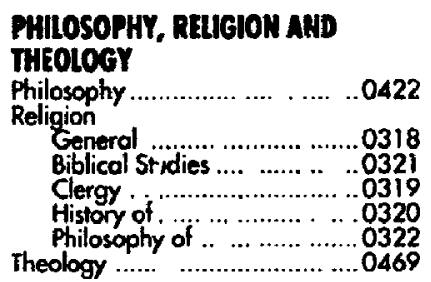

\section{SOCIAL SCIENCES}

American Studies ................ 0323

Anthropology

Cultural ........................ 0326

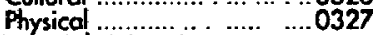

Business Administration

General ..................... 0310

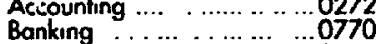

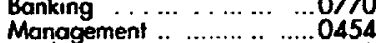

Conadian Shudies

0385

Genero

Agricultural ................ $\quad .0503$

Commerce-Business ..... 0505

Finonce $\ldots . . . \ldots . . . . . . . . .0508$

History. ............... 0509

Lobor ............ 0510

Folktore

0358

Geography.

Herontology

0366

General

0578
Archoeology ............ .. . ..0324

Markefing ...................... 0338

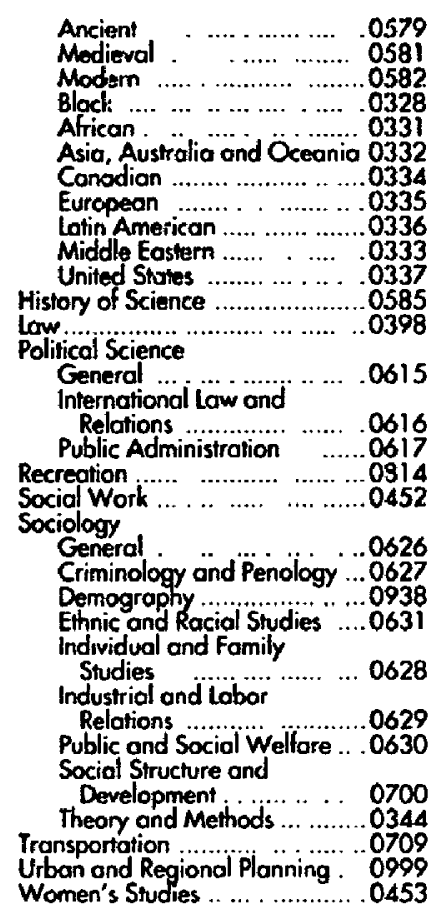

THE SCIINCIS AND ENGINEERING

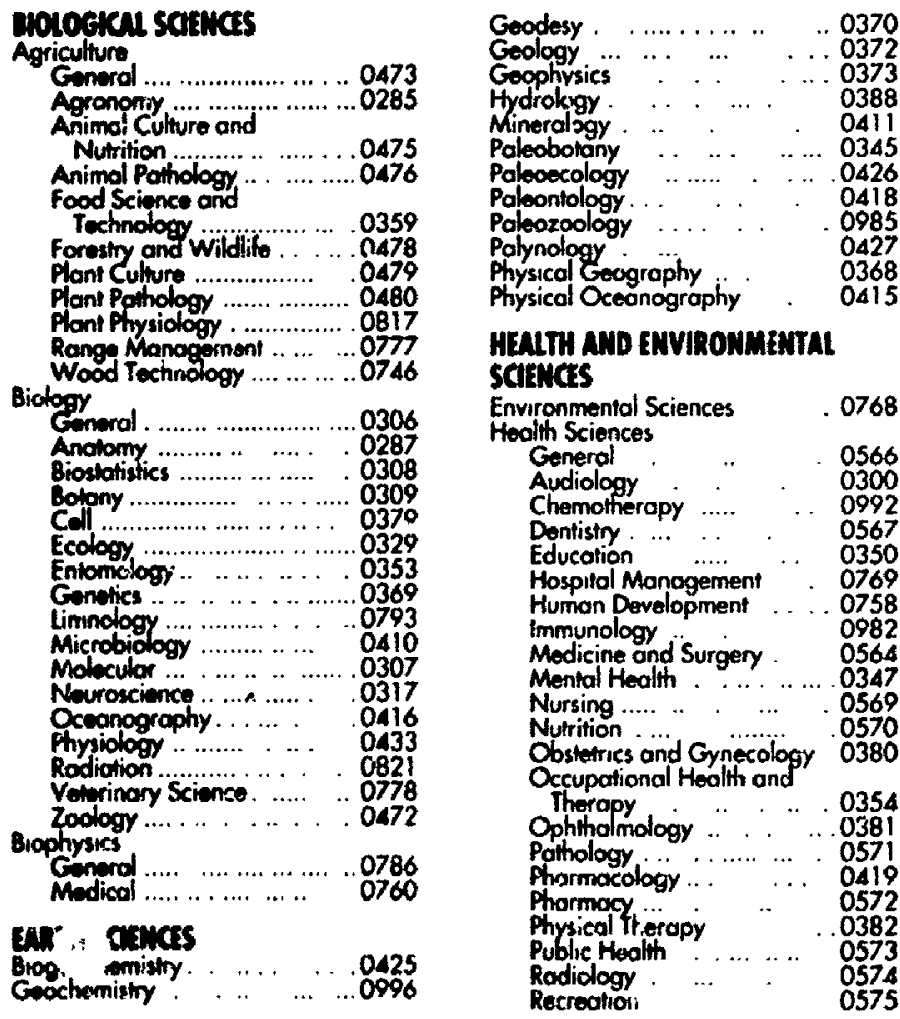

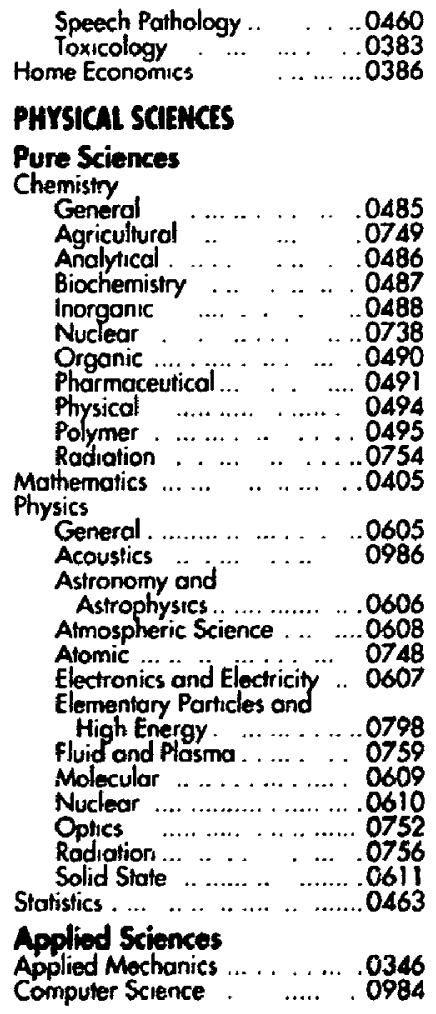

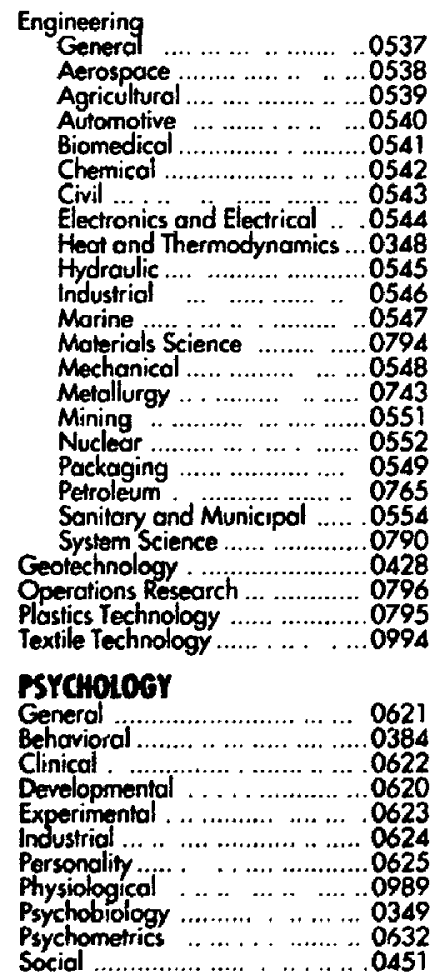

9
2.
31
34
35
36
37
35
98
5
6
7
4
52
26
38
31
28
59
30


The undersigned recommend to the Faculty of Graduate Studies and Research acceptance of the thesis

\section{"SERIES SOLUTIONS OF TIIE \\ LORENZ SYSTEM AND THE DUFFING EQUATION"}

submitted by Andrea L. Zypchen, B.Sc.

in partial fulfilment of the requirements for

the degree of Master of Science

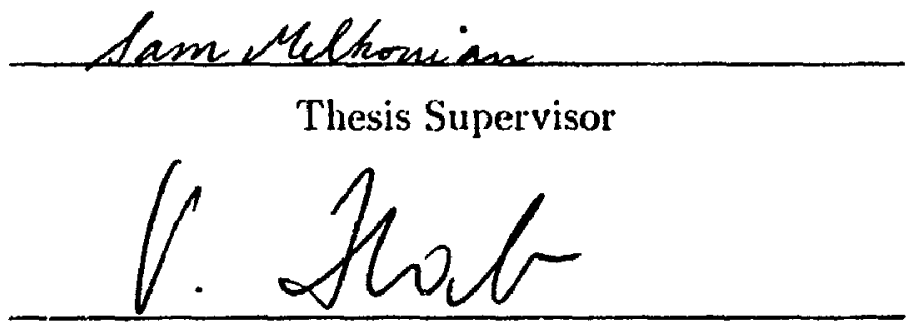

Chair, Department of Mathematics \& Statistics

Carleton University 


\section{Abstract}

Nonlinear differential equations with independent variables in the complex plane may have ordinary, Laurent or psi series solutions. For the Lorenz equations (3dimensional and autonomous) and the Duffing equation (2-dimensional and nonautonomous) these series solutions are formulated, and constructive proofs are given regarding lower bounds on their radii of convergence.

The ordinary series solutions are plotted and compared to plots obtained by numerical integration.

For the psi series solutions of the Lorenz system it was calculated that the lower bound on the radii, when the logarithrnic terms entered at the fourth term, was higher than when the logarithmic terms entered at the second term. The actual radius was observed to follow the same pattern. This is interesting in view of observations made by other researchers [10], that for a different system, the higher the order at which the logarithmic singularities enter, the smaller the chaotic region.

For the psi series solutions of the Duffing equation it was calculated that the lower bounds on the radii decreased as the strength of the forcing increased. This supports observations made by other researchers that as the motion became more chaotic, the singularity pattern condensed and the structures gradually collapsed towards the real axis $[5,7,52,55]$ 


\section{Acknowledgements}

I would like to thank Dr. Sam Melkonian, Ken, Samantha, Nicholas and ray parents. 


\section{Contents}

Acceptance Sheet .................... i ii

Abstract ....................... . . . . . . . . . .

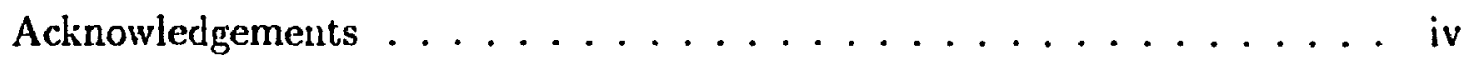

Table of Contents ...................... . vi

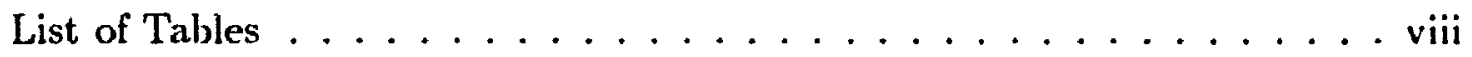

List of Figures . ........................ . . . . .

1 Introduction 1

1.1 The Study of Differential Equations in the Complex Domain . . . . 2

1.2 Results of the Past Decade . . . . . . . . . . . . . . . I

1.3 Series Solutions of Non-linear Differential Equations . . . . . . . 7

1.4 Contents of the Thesis . . . . . . . . . . . . . . . . !

2 The Lorenz System 11

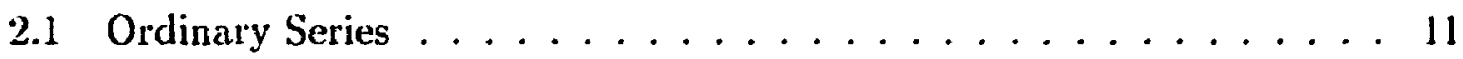

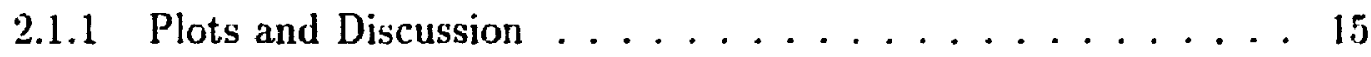

2.2 Laurent Series . . . . . . . . . . . . . . . 17

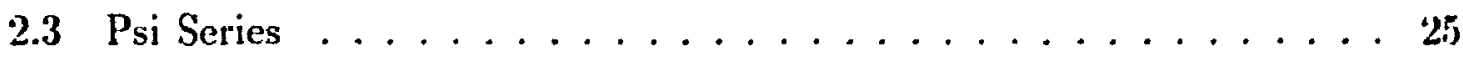

2.3.1 Plots and Discussion .................... 37

3 The Duffing Equation 30

3.1 Ordinary Series . . . . . . . . . . . . . . . . 3! 
3.1.1 Plots and Discussion .................. 42

3.2 laurent Series ........................ . . 43

3.3 Psi Series ....................... 43

$\begin{array}{lll}4 & \text { Figures } & 48\end{array}$

5 Sunmary and Conclusions $\quad 71$ 


\section{List of Tables}

1.1 Differential equations and the types of singularities of their solutions. 3

$2.1 \mathcal{R}_{b b}$ and $\mathcal{R}_{a c t}$ as $R$ varies, for both the $k_{0}=2$ and $k_{00}=4$ rases $\ldots$. 3s 


\section{List of Figures}

4.1 $L(\alpha)$ versus $\alpha$ for the ordinary series solution of the Lorenz system . . 49

$4.2 \mathcal{R}_{\text {est }}$ versus $R$ for the ordinary series solutions of the Lorenz system . 50

4.3 $\mathcal{R}_{\text {est }}$ versus time for the ordinary series solution for $x(t)$ of the Lorenz

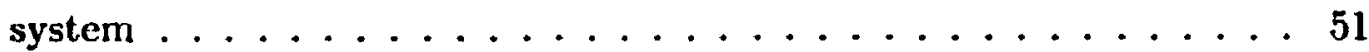

4.4 Ordinary series solution for $x(t)$ of the Lorenz system, truncated at 20 terms, versus time . . . . . . . . . . . . . 52

4.5 Projection of the attractor on the z plane produced by the ordinary series solutions of the Lorenz system . . . . . . . . . . . 53

4.6 $x(t)$ of the Lorenz system versus time, obtained by rumerical integration 54

4.7 Projection of the attractor on the $z$ plane for the Lorenz system, obtained by numerical integration $\ldots \ldots \ldots \ldots \ldots \ldots$

4.8 $x(t)$ versus time for the ordinary series of the Lorenz system, initial conditions $(5,5,5)$ (solid) and $(5.0001,5,5)$ (dashed) $\ldots \ldots \ldots 56$

$4.9 x(t)$ versus time for the ordinary series of the Lorenz system, initial conditions $(5,5,5)$ (solid) and $(5.001,5,5)$ (dashed) . . . . . . 57

$4.10 x(t)$ versus time for the ordinary series of the Lorenz system, initial conditions $(5,5,5)$ (solid) and $(5.01,5,5)$ (dashed) $\ldots \ldots \ldots 58$

$4.11 x(t)$ versus time for the ordinary series of the Lorenz system, initial conditions $(5.5,5)$ (solid) and $(5.1 .5,5)$ (dashed) $\ldots \ldots \ldots \ldots$

$4.12 x(t)$ versus time for the ordinary series of the Lorenz system, initial conditions $(5,5.5)$ (solid) and $(5.2,5,5)$ (dashed) $\ldots \ldots \ldots 60$ 
$4.13 x(t)$ versus time for the ordinary series of the Iorenz system, initial conditions $(-13.9 .-15.856 .932 .13: 33)$ at $:=10.9785$ (dashed) and $(5,5, j)$ at $t=0 \ldots \ldots \ldots \ldots \ldots \ldots$ (il

4.14 Resummation of the double series along the lines $n=k+2 l \ldots \ldots$. .

4.15 Solution of the inequality $\epsilon^{k_{0} s}>2 k+k s \ldots \ldots$ (iis

$4.16 L(\alpha)$ vs $n$ for the psi series solution of the Lorenz system . . . . . . (i.t

4.17 The psi series for $x(t)$ of the Lorenz system versus the truncation point, $t=0.9, t=1.0$ and $t=1.1$ (top to bottom) . . . . . . . (i5)

4.18 The ordinary series for $x(t)$ of the Dulfing equation. truncated at 25 terms, versus time ................... (ifi

4.19 The ordinary series for $x(t)$ versus $x^{\prime}(t)$ of the Dufting equation, truncated at 25 terms .........................

$4.20 x(t)$ of the Duffing equation versus time, obtained by numerical inte-

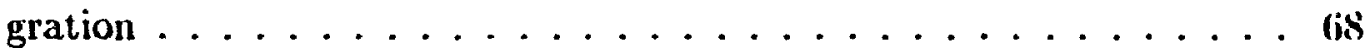

$4.21 x(t)$ versus $x^{\prime}(t)$ of the Dulfing equation. obtained by mumerical inte-

$4.22 x(t)$ versus $t$ for the ordinary series of the Duffing mpuation, initial conditions $(0,0)$ (solid) and $(0.01,0,0)$ (dashed) . . . . . . . 70 


\section{Chapter 1}

\section{Introduction}

Nonlinear phenomena abound in nature. Yet the differential equations which govern their dynamics rarely have an aualytic solution. Numerical integration is often the only resort for estimating the system's trajectories.

Series solutions offer an alternative with several benefits. Firstiy, the series are explicit expressions of the solutions. Secondly, if the independent variables are permitted to assume complex values. much additional information may be gained, including a strong indication of which values of the system's parameters will lead to integrability.

The remainder of the introduction covers the following topics. A brief account is given of how and why the study of differential equations in the complex domain began. Research of the past decade, on the relationship between a system's complex time singularity structure and its real time behaviour, is summarized. Finally, the various types of series solutions are described and the contents of the thesis outlined. 


\subsection{The Study of Differential Equations in the Complex Domain}

In the second half of the nineteenth century, Cauclyy began investigating the nature of differential equations in the complex plane. Instead of attempting to discover global solutions, valid for all initial conditions, researchers became interested in determining local solutions in the complex plane, which could thei be analytically continued to form more general solutions. However, this method did not always succeed, since the ability to analytically continue solutions is very much related to the nature of their singularitics.

Singularities (points at which a solution is not analytic) can be pcies, branch points (algebraic or transcendental (e.g. logarithmic or irrational)), or essential. Each one of these can be fixed or movable. The location of a lixed singularity is determined by the coefficients of the differential equation. 'The location of a movable: singularity is dependent upon the initial conditions. All singularities of linear differential equations are fixed, whereas nonlinear equations may possess either type. All singularities which are not poles are called critical points. Differential equations whose solutions admit various types of singularities are exhibited in 'Table 1.1.

The solution of a differential equation with fixed singularities can be allalytically continued to produce a single-valued, general solution. On the sther hand, if a diffe ential equation possesses movable singularities which are not poles, analytic continuation of its solution may be difficult or impos: ble [39]. With this knowledge, many mathmaticians became interested in classifying ordinary differential equation: according to the types of singularities adınitted by their solutions.

In 1884 Fuchs showed that the only equation of the form $\frac{d x}{d t}=P(x, t)=\frac{\rho^{\prime}(x, t)}{Q(x, t)}$, where $P$ and $Q$ are polynomial in $x$, with coefficients analytic in $t$, which is free from movable critical points. is the generalized Riccati equation

$$
\frac{d x}{d t}=p_{2}(t) \cdot r^{2}+p_{1}(t) x+p_{0}(t) \text {. }
$$

where $p_{2}(t), p_{1}(t)$ and $p_{0}(t)$ are analytic functions [4:3].

In 1888 Painlevé proved that for first order equations of the form $C\left(\frac{d x}{d t}, x, t\right)=0$, 


\begin{tabular}{|l|l|l|}
\hline \hline Differential Equation & Solution & Type of Singularity \\
\hline$w^{\prime}+w^{2}=0$ & $w(z)=\left(z-z_{0}\right)^{-1}$ & movable pole \\
\hline $\begin{array}{l}w^{\prime}-\lambda w^{1-\frac{1}{\lambda}}=0 \\
\lambda \in Q(\text { rational })\end{array}$ & $w(z)=\left(z-z_{0}\right)^{\lambda}$ & movable rational branch point \\
\hline$w^{\prime}-e^{w}=0$ & $w(z)=\ln \left(z-z_{0}\right)$ & movable logarithmic branch point \\
\hline$w^{\prime}+w(\ln w)^{2}=0$ & $\left.w_{i} z\right)=e^{\frac{1}{z-z_{0}}}$ & movable essential singularity \\
\hline $\begin{array}{l}(z-c) w^{\prime}=\lambda w \\
\lambda \in I^{-}\end{array}$ & $w(z)=k(z-c)^{\lambda}$ & fixed pole \\
\hline $\begin{array}{l}(z-c) w^{\prime}=\lambda w \\
\lambda \in Q\end{array}$ & $w(z)=k(z-c)^{\lambda}$ & fixed rational branch point \\
\hline $\begin{array}{l}(z-c) w^{\prime}=\lambda w \\
\lambda \in \bar{Q}(\text { irrational })\end{array}$ & $w(z)=k(z-c)^{\lambda}$ & fixed transcendental branch point \\
\hline$w^{\prime}=\frac{-w}{(z-c)^{2}}$ & $w(z)=k e^{\frac{1}{z-c}}$ & fixed essential singularity \\
\hline \hline
\end{tabular}

Table 1.1: Differential equations and the types of singularities of their solutions

with $G$ polynomial in $\frac{d x}{d t}$ and $x$, and analytic in $t$, the movable singularities are poles and/or algebraic branch points [43].

In 1888 Sophie Kowalevskaya won the Bordin Prize of the Paris Academy of Science for her research on the governing equations of a spinning top (sixth order). Motivated by the work of Fuchs, she determined for which values of the system's parameters, the solutions were free from movable critical points. Of the four sets of parameters she found that met this condition, three sets gave equations which had known solutions. She successfully integrated the equations of motion for the fourth set of parameters $[45,46]$.

In 1897 Painlevé, aiming to answer a question posed by Picard, considered the singularities of all second-order ordinary differential equations (ODEs) of the form

$$
\frac{d^{2} w}{d z^{2}}=F\left(z, w \cdot w^{\prime}\right),
$$

where $F$ is rational in $w^{\prime}$, algebraic in $w$ and anaiytic in $z$. Painlevé, Fuchs, Gambier and Picard found fifty equations whose solutions were free from movable critical 
points $[3 \div, 43]$. Of these, fourty-four were integrable by quadrature or linearization. The remaining six required new transcendental functions (PI - PVI) to be solved.

In 1983 Weiss, Tabor and Carnevale defined a Painlevé property for partial differential equations (PDEs), taking into account the fact that a fuiction of several variables may be singular not at a point, but on a manifold [66].

Further classification of differential equations in terms of their movable singularities has been undertaken by Chazy [21], Garnier $[33]$, Bureau $[14,15,16,17]$ (third-order equations), Ramani et al. [57] (two-dimensional quadratic systems), Hlavatý $[40,41,42]$ and Cosgrove $[23,24,25]$ (PDEs).

\subsection{Results of the Past Decade}

Over the past decade, many observations have been made, a few claims proven, and many conjectures put forth, on the relationship between a system's complex time singularity structure and real time behaviour, which are now summarized.

Ziglin [6S] has proved that transversal intersections of separatrices in nearintegrable Hamiltonian systems with two degrees of freedom imply the existence of infinitely sheeted solutions. This means that for this type of system, with the Painlevé property, there can be no transversal intersections of separatrices (considered a significant cause of chaotic behaviour). Bountis et al. [7] extended Ziglin's results to a particular type of non-llamiltonian system.

There is no proof of a general connection between the Painlevé property and complete integrability of Hamiltonian systems. However. Adler and Mourbeke $[1,2]$ have studied the particular case of Ilamiltonians where the constants of motion (I's) are rational functions of the positions $\left(p_{i}\right.$ 's) and momenta ( $q_{2}$ 's), and the corresponding manifold is part of a torus ${ }^{1}$. In this case, the Hamilton-Jacobi procedure can be utilized and the system is deemed "algebraically integrable" ${ }^{2}$. Their theorem

\footnotetext{
${ }^{1}$ Note that while the Louiville-Arnold theorem shows that when $\mathrm{N}$ I's in involution exist the solutions lie on $\mathrm{N}$ dimensional real tori parametrized by the I's, if $t$ is allowed to be complex, then the manifold is no longer necessarily a torus.

${ }^{2}$ Algebraic integrability is defined by AM as being a property of those systems: which possess a full set of rational integrals and whose motion is linearizable on a complex torus.
} 
states that algebraic integrability implies that the system possesses the Painleve property $[1,2]$. The full converse of this theorem has yet to be proven, but no counterexamples have been found to date, and many examples have $[55,57]$.

Many researchers have experimented with various equations and the Painleve property. The Painleve technique has had unlimited success in determining parameter regions in which a system is integrable $[20,48,51,37,56,35,34,19,28,49,8$, $27,59,30,5,53,65,57,18,11,52,22,54]$ (and references therein).

No ODE has been found that is integrable (has single-valued solutions), yet does not satisfy the Painleve property [29]. The story is different for PDEs. The DymKruskal equation $\left(u_{t}=u^{3} u_{x x x}\right)$, the nonlinear diffusion equation $\left(u_{t}=\left(u^{-2} u_{x}\right)_{x}\right)$, the Wadati-Kohno-Ichikawa equation, and the Bruschi-Ragnisco hierarchy, among others, are solvable by Inverse Scattering (IS), but do not possess the Painlevé property [5.5]. The Dym-Kiruskal cquation, however, does possess the weak Painlevé property (it has rational branch points) and can be transformed into the KDV equation, which does have the Painleve property [57].

What is known about the implications, regarding integrability, of an equation possessing the weak Painlevé property? Bountis et al. have found that those systems which satisfy the weak Painleve property and are integrable have globally finitely sheeted solutions, whereas those which are not integrable have globally infinitely sheeted solutions [3, 4]. For two two-dimensional autonomous Hamiltonian systems possessing the weak Painleve property, it was shown that an appropriate transformation of the dependent and independent variables resulted in a system with the full Painleve property [57]. Sitnikov studied the equation of a periodically driven, one degree of freedom oscillator (non-autonomous). It possesses the weak Painlevé property, yet. when expanded about a fixed singularity, one of the compatibility conditions is not met and logarithmic terms must $b$ introduced. For this case the system is hown to behave chaotically $[57,58]$.

What role $d$. lixed singularitics play in integrability? Will a system with the full Painlevé property. but a fixed non-pole singularity be integrable? Up to second order, these singularities will disappear through linearization. For higher orders the question is unanswered [58]. Fixed singularities can cause non-integrability and 
chaotic behaviour $[4 \pi, 57,58]$.

There are other reasons which indicate that the Painleve property is an inadequate criterion for integrability. There are equations which satisfy the Painleve property only after a suitable transformation [ 47$]$. Movable essential singularities in some cases are compatible with integrability [47]. There is a PDE that can be integrated by quadratures and may have any singularity structure $\left(u_{x t}+u u_{x x}+F\left(u_{x}\right)=0\right)$. Note that when an equation is integrable by quadratures, the singularity structure is irielevant [13]. Logarithmic singularities are not incompatible with integrability (for example, the Lotka-Volterra system) [9].

So what complex singularity structure may signify integrability? The singularity distributions of several nonintegrable systems have been investigated. For systems with logarithmic branch points. the singularities are seen to cluster on infinitely many Riemann sheets in one-armed (ABC Flows [26]), two-armed (Lorenz [65], driven pendulum [53], Morse oscillator [54]) and four-armed (Duffing [30, 57]) structures. For systems with transcendental branc'ı points (the Hénon-Heiles system $[18,20,49]$ and Kuramoto's equation, self-similar spirals of singularities were found, and proven to form a natural boundary, beyond which the solution could not be analytically continued $[18,20,49]$. Ilowever, as the authors warn, there are cases, such as the third-order Chazy equation, where sufficiently nice natural boundaries can lead to integrability.

Interesting correlations between the complex time singularity structures of systems and their real time behaviour have been found. For the Lorenz system, the positions of singularities were found to correlate with the positions of the local maxima of one of the dependent variables. Nlso the amplitudes of the maxima were seen to correlate with the distances of the singularities from the real axis [65]. For Duffing's equation, it was found that as the motion became more chaotic, the singularity pattern condensed and the structures gradually collapsed towards the real axis $[5,7,52,55]$, and the same correlations between the local maxima and the singularity positions were found [i]. For the governing equations of anharmonic oscillators, it was also observed that the higher the order at which the logarithmic singularities enter, the smaller the chaotic region [10]. In addition, correlations have been found 
between the timing of turbulent "bursts" and the real part of complex singularities of equations governing turbulence [31].

As demonstrated by the above results, there is clearly a deep connection between a system's complex time singularity structure and its real time behaviour. It is quite apparent that the requirement that a system have only poles for movable singularities in order to be integrable is too restrictive, and ignores the possible consequerices of essential singularities and fixed critical points. It is interesting to note that Painleve defined a critical point as a point at which the solution was multivalued. He was interested in equations with no critical singularities (fixed or movable) [58].

As this body of research matures, various researchers have proposed new conjectures as to the types of singularity structures which will ensure integrability. T.C. Bountis $e t$ al. [6] have conjectured that the existence of infinitely sneeted solutions in complex time is a necessary condition for non-integrability and chaotic behaviour in real time. M.D. Kiruskal conjectures that a more robust definition of integrability would require that the solutions' possible values not be dense [47]. The validity of these conjectures remains to be tested, and many questions remain.

\subsection{Series Solutions of Non-linear Differential Equa- tions}

Series solutions often provide a means of determining a system's singularity structure, in particular by the following method.

We begin by assuming that the $i^{\text {th }}$ component of the solution is of the form

$$
u_{i}(t)=\sum_{\alpha=0}^{\infty} a_{k}\left(t-t_{0}\right)^{\alpha-p}
$$

where $t_{0}$ is the location of the morable singularity.

The possible values of $p$ can then be calculated by substituting thes series into the system, and balancing the dominant terms.

If $p$ is a negative integer, or zero, $t_{0}$ is no longer a singular and an ordinary series solution will be obtained. For all systems, if an analytic solution exists, it is possible to obtain a balance by setting $p=0$. 
If $p$ is irrational or complex. an appropriate psi series will form the general solution. For a further description of such series and proofs of convergence see the Master s thesis of M.A. Hemmi [36].

If $p$ is a positive integer we then determine the recursion relation for the coetficients of the series. which will be fo the form

$$
B_{\alpha} a_{\alpha}=W_{\alpha}
$$

where $B_{\alpha}$ is a square matrix and $W_{\alpha}$ is determined by the previous $a_{\alpha}$ 's. $a_{a}$ will be arbitrary whenever $B_{c}$ is singular, so long as a compatibility condition is met. The resonances are defined to be these particular values of $\alpha$.

If $p$ is rational, but not integer, it may be possible to make a change of variable so that for the differential equation in the new variable, $p$ is a positive integer. If this transformation is possible, the analysis continues in the new variable, otherwist a psi series is necessary to form a general solution.

If one resonace is -1 and all others are positive integers, and all the compatibility conditions are met, a Laturent series solution will be valid, and the solution will have movable poles.

If not all of the compatibility condtions are inet, a logarithmic psi series must be formed in order to regain the arbitrary coefficients lost by the incompatible resonances. This was discovered by noting that when an ordinary differential equation

$$
y^{\prime \prime}+p(x) y^{\prime}+q(x) y=0
$$

has indicial roots $r_{1}$ and $r_{2}$ which differ by an integer, the Frobenius method states that one solution is

$$
y_{1}(x)=\sum_{n=0}^{\infty} d_{n} x^{n+r_{1}}
$$

and a second linearly independent solution is

$$
y_{2}(x)=A y_{1} \log a+\sum_{n=0}^{\infty} e_{n} s^{n+r_{2}} .
$$

where $A$ is a constant.

If the resonances do not fit this particular pattern an appropriate psi series will be necessary. 
Two things must be noted. Since a general solution to an $n^{\text {th }}$ order differential equation requires $u$ arbitrary parameters, and the location of the singularity $t_{0}$ is arbitrary, these series form general solutions if and only if the number of resonances is equal to the order of the differential equation less one. Secondly, to form a solution thes series must converge within a non-zero radius.

\subsection{Contents of the Thesis}

In this thesis we present the application of the previously described method to the Lorenz (third-order and autonomous) system

$$
\begin{aligned}
& x^{\prime}=\sigma(y-x) \\
& y^{\prime}=-x z+R x-y \\
& z^{\prime}=x y-B z,
\end{aligned}
$$

where $x^{\prime}, y^{\prime}, z^{\prime}$ denote derivatives with respect to time and $\sigma, B$ and $R$ are nonnegative, real-valued parameters of the system, and the Duffing (second-order and nonautonomous) equation

$$
x^{\prime \prime}+k x^{\prime}+p x^{3}=B_{1} F(t)+B_{0},
$$

where $k, p, B_{1}$ and $B_{0}$ are real-valued parameters of the system.

A lower bound on the radius of convergence is estimated for each type of series (ordinary, Laurent, logarithmic psi), solutions are plotted and the truncation error is estimated. Althongh the psi series expansions for the solution riear the singularities of the system have been thought to possibly serve as an explicit solution, to date their convergence is undetermined $[3,18,30,57]$.

All of the proofs which determine a bound on the radius of convergence of the series are similar and are based upon a convergence proof for logarithmic psi series solutions for Emden's equations (second-order and autonomous), carried out by Einar Hille [38]. $A$ bound is first assumed to exist on the first $N-1$ coefficients. It is then shown that for this same bound to hold for the $N$ th and higher coefficients, $N$ must be larger than a certain value, say $N^{*}$. If the bound is calculated based 
on the first $N-1$ coefficients, for $N$ greater than or equal $N^{*}$, the bound will hold for all the coefficients. The ratio test is then applied to the bounding series, which indicates for what values of $t$ the original series will converge.

The behaviour of the ordinary series solutions are compared to those obtained by numerical integration and factors affecting the bound are explored.

All calculations have been executed either in Mathematica or in $\mathrm{C}$. 


\section{Chapter 2}

\section{The Lorenz System}

The Lorenz equations (1.1) are the product of a search by a meteorologist, Edward Lorenz, for a three-dimensional system of ODEs which would demonstrate both the regular and the unpredictable behaviour characteristic of weather [61]. They are a truncated version of an infinite-dimensional system of equations modelling fluid convection in a two dimensional heat cell heated from below. The values of the parameters $\sigma, B$ and $R$ are proportional, respectively, to the Prandtl number, the Rayleigh number and some physical dimensions of the convection cell.

Numerous researchers have studied the dynamical behaviour of the Lorenz uquations, most comprehensively Sparrow [61].

Painlevé analysis has been undertaken by Tabor $[65,60,50]$.

\subsection{Ordinary Series}

About an ordinary point $t_{1}$, power series solutions

$$
x(t)=\sum_{\alpha=0}^{\infty} a_{\alpha} \tau^{\alpha} \cdot y(t)=\sum_{\alpha=0}^{\infty} b_{\alpha} \tau^{\alpha} \text { and } z(t)=\sum_{\alpha=0}^{\infty} c_{\alpha} \tau^{\alpha},
$$

where $\tau=t-t_{1}$, will be valid within the radii of convergence of the series. Using the formula

$$
\left(\sum_{\alpha=0}^{\infty} a_{\alpha} \tau^{\alpha-\alpha_{1}}\right)\left(\sum_{\alpha=0}^{\infty} b_{\alpha} \tau^{\alpha-\alpha_{2}}\right)=\sum_{\alpha=0}^{\infty}\left(\sum_{\beta=0}^{\alpha} a_{\beta} b_{\alpha-\beta}\right) \tau^{\alpha-\left(\alpha_{1}+\alpha_{2}\right)}
$$


and substituting $x(t), y(t)$ and $z(t)$ of this torm into the Lorenz equations (1.1) yields

$$
\begin{aligned}
\sum_{\alpha=0}^{\infty}\left(a_{\alpha}(\alpha)-\sigma b_{\alpha-1}+\sigma a_{\alpha-1}\right) \tau^{\alpha-1} & =0, \\
\sum_{\alpha=0}^{\infty}\left(b_{\alpha}(\alpha)-R a_{\alpha-1}+b_{\alpha-1}+\sum_{\beta=0}^{\alpha-1} a_{\beta} c_{\alpha-1-\beta}\right) \tau^{\alpha-1} & =0, \\
\sum_{\alpha=0}^{\infty}\left(c_{\alpha}(\alpha)-\sum_{\beta=0}^{\alpha-1} a_{\beta} b_{\alpha-1-\beta}+B c_{\alpha-1}\right) \tau^{\alpha-1} & =0,
\end{aligned}
$$

after shifting the indices of summation where necessary to obtain identical powers of $\tau$. Note that termwise differentiation is valid since the uniform convergence of the sum of differentiated terms can be easily proven.

For these equations to be satisfied for all $t$ it is necessary that the coefficient of each power of $\tau$ be zero. Thus we obtain the three recursion relations (RR) in matrix form

$$
\left(\begin{array}{ccc}
\alpha & 0 & 0 \\
0 & \alpha & 0 \\
0 & 0 & \alpha
\end{array}\right)\left(\begin{array}{c}
u_{\alpha} \\
b_{\alpha} \\
c_{\alpha}
\end{array}\right)=\left(\begin{array}{c}
\sigma b_{\alpha-1}-\sigma a_{\alpha-1} \\
R a_{\alpha-1}-b_{\alpha-1}-\sum_{\beta=0}^{\alpha-1} a_{\beta} c_{\alpha-1-\beta} \\
\sum_{\beta=0}^{\alpha-1} a_{\beta} b_{\alpha-1-\beta}-B c_{\alpha-1}
\end{array}\right),
$$

or,

$$
A_{\alpha} \mathbf{v}_{\alpha}=W_{\alpha} .
$$

Since $A_{\alpha}$ is singular only for $\alpha=0, a_{0}, b_{0}$ and $c_{0}$ are the three arbitrary coefficients necessary for these series to constitute a general solution.

Note that throughout the thesis. the norm of an ' by $u$ matrix $A$ is defined as

$$
\|A\|=\max _{1 \leq i \leq n}\left(\sum_{j=1}^{n}\left|a_{i j}\right|\right) \text {. }
$$

In order to prove the existence of a lower bound on the radii of convergence of the three series, we first establish a bound on the coefficients of the series with the following proposition.

Proposition 1 For all $M, 0<M<1$, there exists $K, 1<K<\infty$, such that for all $\alpha \geq 1$

$$
\left\|v_{a}\right\| \leq M K^{a}
$$




\section{Proof.}

The proposition is a consequence of the following

\section{Claim 1 If}

$$
N \geq \max \left(2 \sigma, \frac{R+1+\left|a_{0}\right|+\left|c_{0}\right|}{1-M}, \frac{B+\left|a_{0}\right|+\left|b_{0}\right|}{1-M}\right)
$$

and if

$$
\left\|v_{\alpha}\right\| \leq M K^{\alpha a}
$$

for all $\alpha<N$, then

$$
\left\|\mathbf{v}_{\alpha}\right\| \leq M K^{\alpha}
$$

$\operatorname{for} \alpha=N$

The proposition will then follow by induction, with

$$
K^{\prime}=\max _{1 \leq \alpha \leq N-1} L(\alpha)
$$

where

$$
L(\alpha)=\left(\frac{\left\|\mathbf{v}_{\alpha}\right\|}{M}\right)^{1 / \alpha}
$$

We now prove the claim.

Given that (2.4) holds for $1 \leq \alpha \leq N-1$, for $\alpha=N$ we obtain

$$
\begin{gathered}
\left|a_{\alpha}\right| \leq \frac{\sigma}{\alpha}\left(\left|b_{\alpha-1}\right|+\left|a_{\alpha-1}\right|\right) \\
\leq \frac{2 \sigma}{\alpha} M / k^{\alpha}, \\
\left|b_{\alpha}\right| \leq \frac{1}{\alpha}\left(R\left|a_{i-1}\right|+\left|b_{\alpha-1}\right|+\left(\sum_{\beta=1}^{\alpha-2}\left|a_{\beta}\right|\left|c_{\alpha-1-\beta}\right|\right)+\left|a_{0}\right|\left|c_{\alpha-1}\right|+\left|a_{\alpha-1}\right|\left|c_{0}\right|\right) \\
\leq \frac{1}{\alpha}\left(R M K^{\alpha-1}+M K^{\alpha-1}+M^{2}\left(\sum_{\beta=1}^{u-2} K^{\beta} K^{\alpha-1-\beta}\right)+\left|a_{0}\right| M K^{\alpha-1}+\left|c_{0}\right| M K^{\alpha-1}\right) \\
\left.\leq \frac{1}{\alpha}\left(R+1+\left|a_{0}\right|+\left|c_{0}\right|\right) M K^{\alpha-1}+M^{2} K^{\alpha-1}\left(\sum_{\beta=1}^{\alpha-2} 1\right)\right) \\
\leq \frac{1}{\alpha}\left(R+1+M \alpha+\left|a_{0}\right|+\left|c_{0}\right|\right) \cdot M K^{\alpha}
\end{gathered}
$$


and

$$
\begin{aligned}
& \left|c_{\alpha}\right| \leq \frac{1}{\alpha}\left(\left(\sum_{j=1}^{\alpha-2}\left|a_{a}\right|\left|b_{a-1-13}\right|\right)+\left|a_{0}\right|\left|b_{a n-1}\right|+\left|a_{\alpha-1}\right|\left|b_{0}\right|+|b| c_{x-1} \mid\right) \\
& \leq \frac{1}{\alpha}\left(M^{2}\left(\sum_{i=1}^{a-2} K^{-3} K^{\alpha-1-i}\right)+\left|u_{0}\right| \cdot M K^{\alpha-1}+\left|b_{0}\right| \cdot M K^{\alpha-1}+B . M K^{i \alpha-1}\right) \\
& \leq \frac{1}{\alpha}\left(\left(B+\left|a_{0}\right|+\left|b_{0}\right|\right) M h^{\alpha-1}+M^{2} k^{\alpha-1}\left(\sum_{j=1}^{\alpha-2} 1\right)\right) \\
& \leq \frac{1}{\alpha}\left(B+\left|a_{0}\right|+\left|b_{0}\right|+M \alpha\right) M K^{-\alpha} \text {. }
\end{aligned}
$$

Since, for $\alpha \geq 1,1_{1}$ is nonsingular. we obtain

$$
\left\|v_{\alpha}\right\| \leq\left\|A_{\alpha}\right\|\left\|G_{\alpha}\right\| M K^{\alpha}=\frac{1}{\alpha}\left\|G_{\alpha}\right\| M K^{\alpha}
$$

where

$$
G_{\alpha}=\left(\begin{array}{c}
2 \sigma \\
R+1+\left|a_{0}\right|+\left|c_{0}\right|+M \alpha \\
B+\left|a_{0}\right|+\left|b_{0}\right|+M \alpha
\end{array}\right) .
$$

In order for the bound to hold we must have, for $\alpha=N$,

$$
\frac{\left\|i_{\alpha}\right\|}{\alpha} \leq 1
$$

or equivalently,

$$
\mathrm{V} \geq \max \left(2 \sigma . \frac{R+1+\left|a_{0}\right|+\left|c_{0}\right|}{1-M}, \frac{B+\left|\boldsymbol{a}_{0}\right|+\left|b_{0}\right|}{1-M}\right),
$$

and the claim and thus tha proposition are proved.

We are now prepared to state and prove the theorem regarding a lower bound on the radii of convergence of the three scries.

Theorem 1 There exists $\mathcal{R}, 0<\mathcal{R}<\infty$, such that for $|\tau|<\mathcal{R}$, the series

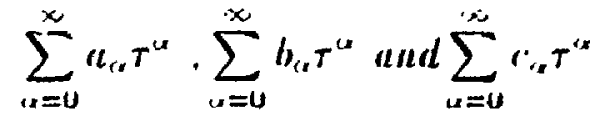

converge absolutely for all values of the system s parameters $\sigma, B$ and $R$. 
Proof. Ujing Proposition 1 the theorem follows easily from the comparison test.

Considering the series for $\alpha \geq 1$ we have

$$
\sum_{\alpha=1}^{\infty} \mathbf{v}_{\alpha} \tau^{\alpha} \leq\left\|\sum_{\alpha=1}^{\infty} \mathbf{v}_{\alpha} \tau^{\alpha}\right\| \leq \sum_{\alpha=1}^{\infty}\left\|\mathbf{v}_{\alpha}\right\||\tau|^{\alpha} \leq \sum_{\alpha=1}^{\infty} M K^{\alpha \alpha}|\tau|^{\alpha} .
$$

Applying the ratio test to this last series gives

$$
\lim _{\alpha \rightarrow \infty}\left(\frac{K^{\alpha+1}}{K^{\alpha}}|\tau|\right)=K|\tau|
$$

For the bounding series to converge we must have $K|\tau|<1$ or $|\tau|<\frac{1}{K}$. Therefore the ordinary series converge for

$$
\mathcal{R} \geq \frac{1}{\Lambda^{\prime}}=\mathcal{R}_{l b}
$$

where $\mathcal{R}_{l b}$ denotes the estimated lower bound on the radii of convergence of the series.

\subsubsection{Plots and Discussion}

In this section, for specific parameter values, $\mathcal{R}_{b b}$ will be calculated and the solution plotted by repeatedly re-expanding the series about a point within the rarius of convergence.

For the initial conditions $(x(0), y(0), z(0))=(5,5,5)$ and the parameter values $\sigma=10, B=\frac{8}{3}$ and $R=28, \mathcal{R}_{l b}$ is entirely determined by $\left|b_{1}\right|$ since the maximum of $L(\alpha)$ occurs at $\alpha=1$ (see Figure 4.1), and $\left|b_{1}\right|$ is the maximum of $\left|a_{1}\right|,\left|b_{1}\right|$ and $\left|c_{1}\right|$. Therefore, as $M \rightarrow 1$. $\mathcal{R}_{l b} \rightarrow \frac{1}{\left|b_{1}\right|}=0.01$.

Note that $L(\alpha)$ appears to be converging to 5 which is approximately $\frac{1}{R_{a c t}}$. This is what Hadamard's formula lor the radius [44]

$$
\mathcal{R}=\frac{1}{\lim \sup _{\alpha \rightarrow \infty}\left|a_{\alpha}\right|^{\frac{1}{\alpha}}}
$$

predicts should occur as $M$ approaches 1 . This formula gives the radius as approxnately 0.2. 
A plot of $\mathcal{R}_{l b}$ versus $R$. the system parameter usually varied in dynamical studies is shown in Figure 4.2. For all values of $R$ the maximum of $L(\alpha)$ occurs at $\alpha=1$ and $K$ equals $\left|b_{1}\right|$. The value of $\left|b_{1}\right|$ is determined by $R$ and the initial conditions. Therefore as $R$ increases, the value of the estimated radius decreases.

A plot of $\mathcal{R}_{l b}$ versus time (Figure 4.3 ) indicates that as the solution moves farther from the axis, the radius decreases. This is as expected, since $\left|a_{0}\right|$ is increasing, increasing $\left|b_{1}\right|$.

Plots of the trajectory defined by $x(t)$ and of a projection of the attractor on the $z$ plane are shown in Figure 4.4 and Figure 4.5. Figure 4.6 and Figure 4.7 show the corresponding plots obtained by numerical integration for the same initial conditions and parameter values, which are identical to those obtained by the series solutions.

The series of plots. Figure 4.8 , Figure 4.9. Figure 4.10 , Figure 4.11 and Figure 4.12 illustrate how the point of divergence of the neighbouring solutions varies with the initial conditions. As the difference in initial conditions increases, the trajectories diverge sooner. It is interesting to note that although these plots are of chaotic motion, it is not due to real time singularities. However, as we shall see in the section on psi series, for these parameter values, logarithmic singularities exist in the complex domain.

Figure 4.13 shows the trajectory of $x(t)$ with the initial conditions $(x(0), y(0), z(0))=$ $(5,5,5)$, and a trajectory which has been perturbed slightly from this solution, at a time just prior to the divergence of the two trajectories from Figure 4.10. The "perturbed" trajectory diverges from the original trajectory (solid line) at a time greater than the previous point of divergence $(t \doteq 11)$. 'This illustrates that divergence does not occur at a specific time, but rather that any two trajectories which begin "close" to each other. are bound to diverge after a sufficient amount of time has elapsed.

Although we can observe that the maximum of $L(\alpha)$ occurs at $\alpha=1$, it does not appear possible to prove this. Therefore. the proof requires that $k$ be calculated based on the first $N-1$ coefficients. where $N$ is determined from (2.5). 'Typical values of $N$ fall within the range of 6 to 300 . Note that since $M$ can be chosen 
between zero and one. the value of $N$ can be decreased by decreasing $M$, thus reducing the amount of calculation required to determine $k$.

It is also interesting to note that since $L(\alpha)$ is a decreasing function, in order to better estimate the lower bound on the radii, the claim (2.4) could be made for $\alpha$ greater than an arbitrary value, say $\hat{\alpha}$. $A$ s $\hat{\alpha}$ tended to infinity, $L(\alpha)$ would tend to $\frac{1}{\mathcal{K}_{\mathrm{ace}}}$. However, this modification $w$ uld greatly increase the value of $N$ required to prove the claim.

Lastly, we estimate the truncation error. Given that

$$
\sum_{\alpha=0}^{\infty} \mathrm{v}_{\alpha} \tau^{\alpha} \leq\left\|\sum_{\alpha=0}^{\infty} \mathrm{v}_{\alpha} \tau^{\alpha}\right\| \leq \sum_{\alpha=0}^{\infty}\left\|\mathrm{v}_{\alpha}\right\||\tau|^{\alpha} \leq \sum_{\alpha=0}^{\infty} \cdot \| K^{\alpha}|\tau|^{\alpha}=M \sum_{\alpha=0}^{\infty}(K|\tau|)^{\alpha}
$$

and within $\mathcal{R}_{l b}, K^{\prime}|\tau|<1$, the truncation error

$$
\begin{aligned}
E_{n} & =\frac{S_{\infty}-S_{n}}{S_{\infty}} \\
& \leq(K|\tau|)^{n+1}
\end{aligned}
$$

where $S_{n}$ is the $n$th partial sum of the geometric series.

The plots of the trajectory of $x(t)$ are insensitive to the point, of truncation, as long as a minimum of eight terms are included. This corresponds to an accuracy of approximately four decimal places.

\subsection{Laurent Series}

In this section the Painleve test is applied to the Lorenz system, and the parameter values for which the Laurent series is valid are determined.

We first determine the leading order behaviour close to a singularity by assuming the solutions are of the form

$$
\begin{aligned}
& x(t)=a_{0} \tau^{\psi}, \\
& y(t)=b_{0} \tau^{3}, \\
& z(t)=c_{0} \tau^{\gamma},
\end{aligned}
$$

where $\tau=\left(t-t_{0}\right), t_{0}$ is the location of the arbitrary singularity, and $t \in \mathbf{C}$. 
Substitution of (2.10) into (1.1) yields

$$
\begin{aligned}
& a_{0} \alpha \alpha \tau^{i^{\prime}-1}=\sigma\left(b_{0} \tau^{3}-a_{0} \tau^{t^{4}}\right) . \\
& b_{0} \beta \tau^{\mu-1}=-a_{0} c_{0} \tau^{v^{2+\gamma}}+R u_{0} \tau^{\prime \prime}-b_{0} \tau^{3} . \\
& c_{0} \gamma \tau^{\gamma-1}=a_{0} b_{0} \tau^{\psi+1}-B c_{0}-B c_{0}^{\gamma} .
\end{aligned}
$$

Balancing the leading orders for equation $(2.11)\left(\tau^{\psi-1}\right.$ and $\left.\tau^{3}\right)$ yields

$$
\begin{aligned}
\psi-1 & =\beta, \\
a_{0} \dot{\psi} & =\sigma b_{0} .
\end{aligned}
$$

Balancing the leading orders for equation (2.12) $\left(\tau^{\psi+\gamma}\right.$ and $\left.\tau^{j-1}\right)$ yields

$$
\begin{aligned}
\beta-1 & =\psi+\gamma, \\
b_{0} \beta & =-a_{0} c_{0} .
\end{aligned}
$$

Balancing the leading orders for equation $(2.13)\left(\tau^{\gamma-1}\right.$ and $\left.\tau^{\psi+13}\right)$ yields

$$
\begin{aligned}
\gamma-1 & =\psi+\beta, \\
c_{\theta} \gamma & =a_{0} b_{0} .
\end{aligned}
$$

The solution of these six equations is $\psi=-1, \beta=-2, \gamma-2$ and

$$
\begin{aligned}
& a_{0}= \pm 2 i, \\
& b_{0}=\mp \frac{2 i}{\sigma}, \\
& c_{0}=-\frac{2}{\sigma},
\end{aligned}
$$

or $a_{0}, b_{0}, c_{0}$ equal to 0 . In the latter case $b_{1}$ and $c_{1}$ are calculated to be zero and $a_{1}, b_{2}$ and $c_{2}$ are arbitrary. These are the initial conditions $x(0), y(0)$ and $z(0)$, respectively, and $t_{0}$ is no longer a singularity. In other words, the Laurent series becomes an ordinary series, as in the last section.

Using this information, the solutions are assumed to he of the form

$$
\begin{aligned}
& x(t)=\sum_{x=0}^{\infty} a_{v} \tau^{u-1}, \\
& y(t)=\sum_{x=0}^{\infty} b_{x} \tau^{x-2}, \\
& z(t)=\sum_{s=0}^{\infty} c_{x} \tau^{u-2} .
\end{aligned}
$$


Substitution of $(2.11)$ into the Lorenz system (1.1) gives

$$
\begin{aligned}
& \sum_{\alpha=0}^{\infty} a_{\alpha}(\alpha-1) \tau^{\alpha-2}=\sigma\left(\sum_{u=0}^{\infty} b_{\alpha} \tau^{\alpha-2}-\sum_{\alpha=0}^{\infty} a_{n} \tau^{\alpha-1}\right) \\
& \sum_{\alpha=0}^{\infty} b_{\alpha}(\alpha-2) \tau^{\alpha-3}=R \sum_{\alpha=0}^{\infty} a_{\alpha} \tau^{\alpha-1}-\sum_{\alpha=0}^{\infty} b_{\alpha} \tau^{\alpha-2}-\left(\sum_{\alpha=0}^{\infty} a_{\alpha} \tau^{\alpha-1}\right)\left(\sum_{\alpha=0}^{\infty} c_{\alpha} \tau^{\alpha-2}\right), \\
& \sum_{\alpha=0}^{\infty} c_{\alpha}(\alpha-2) \tau^{\alpha-3}=\left(\sum_{\alpha=0}^{\infty} a_{\alpha} \tau^{\alpha-1}\right)\left(\sum_{\alpha=0}^{\infty} b_{\alpha} \tau^{\alpha-2}\right)-B \sum_{\alpha=0}^{\infty} c_{\alpha} \tau^{\alpha-2}
\end{aligned}
$$

we obtain

$$
\begin{aligned}
\sum_{\alpha=0}^{\infty}\left(a_{\alpha}(\alpha-1)-\sigma b_{\alpha}+\sigma a_{\alpha-1}\right) \tau^{\alpha-2} & =0 \\
\sum_{\alpha=0}^{\infty}\left(b_{\alpha}(\alpha-2)-R a_{\alpha-2}+b_{\alpha-1}+\sum_{\beta=1}^{\alpha-1} a_{\beta} c_{\alpha-\beta}+a_{0} c_{\alpha}+c_{0} a_{\alpha}\right) \tau^{\alpha-3} & =0 \\
\sum_{\alpha=0}^{\infty}\left(c_{\alpha}(\alpha-2)-\sum_{\beta=1}^{\alpha-1} a_{\beta} b_{\alpha-\beta}-a_{0} b_{\alpha}-b_{0} a_{\alpha}+B c_{\alpha-1}\right) \tau^{\alpha-3} & =0
\end{aligned}
$$

after shifting the indices of summation where necessary, to obtain identical powers of $\tau$.

For these equations to be satisfied for all $\tau$ it is necessary that the coefficient of each power of $\tau$ be zero. Thus we obtain the three re.:sion relations (RR)

$$
\begin{aligned}
a_{\alpha}(\alpha-1)-\sigma b_{\alpha}+\sigma a_{\alpha-1} & =0, \\
b_{\alpha}(\alpha-2)-R a_{\alpha-2}+b_{\alpha-1}+\sum_{\beta=1}^{\alpha \alpha-1} a_{\beta} c_{\alpha-3}+a_{0} c_{\alpha}+c_{0} a_{\alpha} & =0, \\
c_{\alpha}(\alpha-2)-\sum_{\beta=1}^{\alpha-1} a_{\beta} b_{\alpha-\beta}-a_{0} b_{\alpha}-b_{0} a_{\alpha}+B c_{\alpha-1} & =0,
\end{aligned}
$$

where $a_{-1}=a_{-2}=b_{-1}=c_{-1}=0$. Alternatively, in matrix form, we have

$$
\left(\begin{array}{ccc}
(\alpha-1) & -\sigma & 0 \\
-\frac{2}{\sigma} & (\alpha-2) & \pm 2 i \\
\pm \frac{2 i}{\sigma} & \mp 2 i & (\alpha-2)
\end{array}\right)\left(\begin{array}{c}
a_{\alpha} \\
b_{\alpha} \\
c_{\alpha}
\end{array}\right)=\left(\begin{array}{c}
-\sigma a_{\alpha-1} \\
R a_{\alpha-2}-b_{\alpha-1}-\sum_{\beta=1}^{\alpha-1} a_{\beta} c_{\alpha-\beta} \\
\sum_{\beta=1}^{\alpha-1} a_{\beta} b_{\alpha-\beta}-B c_{\alpha-1}
\end{array}\right),
$$

or,

$$
A_{\alpha} v_{\alpha}=W_{\alpha}=\left(\begin{array}{c}
W_{\alpha 1} \\
W_{\alpha 2} \\
W_{\alpha 3}
\end{array}\right)
$$


Note that $A_{\alpha}$ is singliar at $a=2$ and $a=4$. If the two sets of equations defined by $A_{\alpha} \mathbf{v}_{\alpha}=W_{c}$ are not inconsistent for these two values of $a$. there will be an intinite number of solutions for each system. This will provide us with the required further two arbitrary coefficients (in addition to the location of the moveable singularity. $\left.t_{0}\right)$.

The Freedholm Alternative Theorem states under which conditions a singular nonhomogeneous system of equations will be inconsistent or have infinitely many solutions. These conditions supply the compatibility conditions described in Siction 1.3. The theorem states: Given the system $A_{\alpha} v_{18}=W_{1 x}$, where $A_{1 r}$ is singular, there exist infinitely many solutions if and only if $V_{x} \cdot V_{x}=0$, for all $V_{x}$ such that $A_{\alpha}{ }^{T} V_{\alpha}=0$.

For $\alpha=2$,

$$
\begin{gathered}
V_{2}=\left(\begin{array}{c}
\mp 2 i / \sigma \\
0 \\
1
\end{array}\right) \text { and } \\
V_{2}=\left(\begin{array}{c}
-\sigma a_{1} \\
-a_{1} c_{1}+R a_{0}-b_{1} \\
a_{1} b_{1}-B c_{1}
\end{array}\right) .
\end{gathered}
$$

Given that

$$
\left(\begin{array}{l}
a_{1} \\
b_{1} \\
c_{1}
\end{array}\right)=\left(\begin{array}{c} 
\pm \frac{1}{3}(3 \sigma-1-2 \beta) \\
\pm 2 i \\
\frac{2}{3 \sigma}(3 \sigma+1-B)
\end{array}\right) \text {. }
$$

imposing $V_{2} \cdot W_{2}=0$ yiclds the first compatibility condition, namely,

$$
B(B-1)=6\left(j \sigma^{2}-2 \sigma-B \sigma .\right.
$$

This concurs with the compatibility condition found by Thaber and Weiss [50]. When this condition is met the system $A_{2} v_{2}=W_{2}$ will not be inconsistent and will have infinitely many solutions. Thus for values of $\sigma, B$ and $R$ satisfying (2.16), one of $\alpha_{2}$, $b_{2}$ and $c_{2}$ can be made arbitrary. 
For $\alpha=4$

$$
V_{1}=\left(\begin{array}{c}
0 \\
\pm i \\
1
\end{array}\right)
$$

and

$$
W_{4}=\left(\begin{array}{c}
-\sigma a_{3} \\
-a_{1} c_{3}-a_{2} c_{2}-a_{3} c_{1}+R a_{2}-b_{3} \\
a_{1} b_{3}+a_{2} b_{2}+a_{3} b_{1}-B c_{3}
\end{array}\right) .
$$

Taking $a_{2}$ to be arbitrary, we have

$$
\begin{gathered}
b_{2}=a_{2} / \sigma+a_{1}, \\
c_{2}=\mp i a_{2} / \sigma \pm i\left(a_{1} c_{1}-R a_{0}+b_{1}\right) / 2
\end{gathered}
$$

and

$$
\begin{gathered}
\left(\begin{array}{l}
a_{3} \\
b_{3} \\
c_{3}
\end{array}\right)=\left(\begin{array}{c} 
\pm \frac{-i-9, B-6 i B^{2}+16 i B^{3}-3 i \sigma+24 i B \sigma-102 i B^{2} \sigma-54 i B R \sigma+45 i \sigma^{2}+171 i B \sigma^{2}-81 i \sigma^{3}}{108} \\
\pm \frac{-i-9) i B-62 B^{2}+16 i B^{3}-3 i \sigma+24 i B \sigma-102 i B^{2} \sigma-54 i B R \sigma+45 i \sigma^{2}+171 i B \sigma^{2}-81 i \sigma^{3}}{54 \sigma} \\
\frac{(1-B+3 \sigma)\left(1+36 i \sigma 2+4 B+4 B^{2}-6 \sigma-12 B \sigma+9 \sigma^{2}\right)}{54 \sigma}
\end{array}\right)+ \\
\left(\begin{array}{c}
\frac{12}{2}(1-B-3 \sigma) \\
\frac{12}{\sigma}(1-B+2 \sigma) \\
0
\end{array}\right) .
\end{gathered}
$$

Imposing $V_{4} \cdot W_{4}=0$ yiclds

$$
a_{3} c_{1}+a_{2} c_{2}+a_{1} c_{3}-R a_{2}+b_{3}+i a_{3} b_{1}+i a_{2} b_{2}+i a_{1} b_{3}-i B c_{3}=0,
$$

and upon the substitution of $a_{\alpha x}, b_{\alpha x}$ and $c_{c x}, \alpha=1,2,3$, we obtain

$$
\begin{aligned}
& 54 \sigma^{4}-(144 B-99) \sigma^{3}+\left(27 B R+129 B^{2}+156 B+39\right) \sigma^{2}- \\
& \left(\left(9 B^{2}+45 B\right) R+47 B^{3}+8 i B^{2}-27 B+1\right) \sigma+6 B^{4}+13 B^{3}-9 B^{2}-9 B-1+ \\
& \mathbf{a}_{2}\left(5 \cdot 4 i \sigma^{2}+(144 i-171 i B) \sigma+45 B^{2}+9 B-54\right)=0 .
\end{aligned}
$$

$a_{2}$ is arbitrary therefore the coefficient of $a_{2}$ inust vanish, yielding the second compatibility condition

$$
51 \sigma^{2}+(144-1 \pi 1 B) \sigma+45 B^{2}+9 B-54=0 .
$$


The remainder of the terms in (2.1i) must also vanish, yielding the third compatibility condition

$$
\begin{array}{r}
54 \sigma^{4}-(14+B-99) \sigma^{3}+\left(27 B R+129 B^{2}+156 B+39\right) \sigma^{2}- \\
\left(\left(9 B^{2}+45 B\right) R+47 B^{3}+87 B^{2}-27 B+1\right) \sigma+ \\
6 B^{4}+13 B^{3}-9 B^{2}-9 B-1=0 .
\end{array}
$$

The three compatibility conditions (2.16), (2.18), and (2.19) hold for the following values of $\sigma, B$ and $R$

$$
\begin{array}{rlll}
\text { i) } & \sigma=1 / 2 & B=1 & R=0, \\
\text { ii) } & \sigma=1 & B=2 & R=1 / 9, \\
\text { iii) } & \sigma=1 / 3 & B=0 & R \text { arbitrary . }
\end{array}
$$

For these three sets of parameter values the Lorenz system is completely integrable in terms of elliptic functions and Painleve transcendants [50].

In order to prove the existence of a lower bound on the radii of convergence of the three series, we first establish a bound on the coefficients of the series with the following proposition, in exactly the same fashion as for the ordinary series.

Proposition 2 For all $M, 0<. l<1$, there exists $K .1<K<\infty$ such that for all $\alpha \geq 5$

$$
\left\|\mathbf{v}_{t}\right\| \leq M \boldsymbol{K}^{* *}
$$

Proof.

The proposition is a consequence of the following

Assertion 1 If $N$ is such that $Q(N) \geq 0$, where

$Q(\alpha)=\alpha^{3}(1-M)-\alpha^{2}\left(j+J+M\left(\frac{2}{\sigma}-10\right)\right)+\alpha\left(2-J\left(\frac{2}{\sigma}-1\right)+M\left(\frac{10}{\sigma}\right)\right)+x\left(1+\frac{J-9}{\sigma}\right.$

where

$J=\max \left(R+1+M(\alpha-9)+\sum_{i \beta=1}^{1}\left(\left|a_{i \beta}\right|+\left|c_{i \beta}\right|\right), B+M(\alpha-9)+\sum_{\beta=1}^{4}\left(\left|a_{i j}\right|+\left|b_{\beta}\right|\right)\right)$, 
and if

$$
\left\|v_{o r}\right\| \leq M K^{\alpha}
$$

for all $\alpha<N$, then

$$
\left\|\mathrm{v}_{\alpha}\right\| \leq M K^{\alpha} .
$$

for $\alpha=N$.

The proposition will then follow by induction if $N$ is chosen such that $Q(\alpha) \geq 0$ for all $\alpha \geq N$, and

$$
K=\operatorname{mix}_{5 \leq \alpha \leq N-1} L(\alpha),
$$

where

$$
L(\alpha)=\left(\frac{\left\|\mathbf{v}_{o}\right\|}{M}\right)^{\frac{1}{\alpha}} .
$$

We now prove the claim.

Given that (2.21) holds for $5 \leq a \leq N-1$, for $\alpha=N$ we obtain

$$
\begin{gathered}
\left|W_{\alpha 1}\right| \leq \sigma\left|a_{\alpha-1}\right| \\
\leq \sigma M K^{\alpha} \\
\left|W W_{\alpha \cdot 2}\right| \leq R\left|a_{\alpha-2}\right|+\left|b_{\alpha-1}\right|+\sum_{\beta=5}^{\alpha-5}\left|a_{\beta}\right|\left|c_{\alpha-\beta}\right|+ \\
\sum_{\beta=1}^{4}\left|a_{\beta}\right|\left|c_{\alpha-\beta}\right|+\sum_{\beta=\alpha-4}^{\alpha-1}\left|a_{\beta}\right|\left|c_{\alpha-\beta}\right| \\
\leq\left(R+1+M(\alpha-9)+\sum_{\beta=1}^{4}\left(\left|a_{\beta}\right|+\left|c_{\beta}\right|\right)\right) M K^{\alpha}
\end{gathered}
$$

and

$$
\begin{aligned}
\left|W_{n 3}\right| \leq & \sum_{\beta=5}^{\alpha-5}\left|a_{\beta}\right|\left|b_{1,-\beta}\right|+\sum_{\beta=1}^{4}\left|a_{\beta}\right|\left|b_{\alpha-\beta}\right|+ \\
& \sum_{\beta=\alpha-4}^{\alpha-1}\left|a_{\beta}\right|\left|b_{\alpha-\beta}\right|+B\left|c_{\alpha-1}\right| \\
\leq & \left(B+M(\alpha-9)+\sum_{\beta=1}^{4}\left(\left|a_{\beta}\right|+\left|b_{\beta}\right|\right)\right) M K^{\alpha} .
\end{aligned}
$$


Since for $\alpha \geq 5 A_{\alpha}$ is nonsingular,

$$
\left\|\mathbf{v}_{\alpha}\right\|=\left\|A_{\alpha}^{-1} \cdot\right\| V_{\alpha}\|\leq\| \cdot A_{\alpha}{ }^{-1}\|\| G_{\alpha}\|\cdot\| K^{-\alpha}
$$

where

$$
G_{\alpha}=\left(\begin{array}{c}
\sigma \\
R+1+M(\alpha-9)+\sum_{\beta=1}^{1}\left(\left|a_{\beta}\right|+\left|c_{\beta}\right|\right) \\
B+M(\alpha-9)+\sum_{\beta=1}^{\prime}\left(\left|a_{\beta}\right|+\left|b_{\beta}\right|\right)
\end{array}\right)=\left(\begin{array}{c}
G_{\alpha 1} \\
G_{\alpha 2} \\
G_{\alpha 3}
\end{array}\right)
$$

Given that

$$
A_{\alpha}^{-1}=\frac{1}{(\alpha+1)(\alpha-2)(\alpha-4)}\left(\begin{array}{ccc}
\alpha(\alpha-4) & \sigma(\alpha-2) & -2 i \sigma \\
\frac{2}{\sigma}(\alpha-4) & (\alpha-1)(\alpha-2) & -2 i(\alpha-1) \\
\frac{-2 i}{\sigma}(\alpha-4) & 2 i(\alpha-2) & \alpha(\alpha-3)
\end{array}\right) \text {, }
$$

for $\alpha \geq 5$,

$$
\begin{aligned}
\left\|A_{\alpha}^{-1}\right\| & =\frac{\max \left(\alpha^{2}+\alpha(\sigma-4) \alpha^{2}+\alpha\left(\frac{2}{\sigma}-1\right)-\frac{\alpha}{\sigma}\left(\alpha^{2}+\alpha\left(\frac{2}{\sigma}-1\right)-\frac{\alpha}{a}-4\right)\right.}{(\alpha+1)(\alpha-2)(\alpha-4)} \\
& =\frac{\max \left(\alpha^{2}+\alpha(\sigma-4), \alpha^{2}+\alpha\left(\frac{2}{\sigma}-1\right)-\frac{\alpha}{\sigma}\right)}{(\alpha+1)(\alpha-2)(\alpha-4)} \\
& =\frac{\alpha^{2}+\alpha\left(\frac{2}{\sigma}-1\right)-\frac{\alpha}{a}}{(\alpha+1)(\alpha-2)(\alpha-4)},
\end{aligned}
$$

since $\alpha(\alpha-4)<\alpha\left(\frac{2}{\sigma}-1\right)-\frac{8}{\sigma}$ for all $\sigma<3$.

It can also easily be shown that for all of the parameter values $(2.20),\left\|G_{\alpha}\right\|$ is equal to either $G_{\alpha 2}$ or $G_{\alpha 3}$. Therefore $\left\|G_{\alpha}\right\|=J+M(\alpha-9)$ where

$J=\max \left(R+1+M(\alpha-9)+\sum_{j=1}^{4}\left(\left|a_{i j}\right|+\left|c_{\beta}\right|\right), B+M(\alpha-9)+\sum_{\beta=1}^{4}\left(\left|a_{\beta}\right|+\left|b_{\beta}\right|\right)\right)$.

In order that (2.21) hold we must have

$$
\left\|A_{\alpha}^{-1}\right\|\left\|G_{s x}\right\| \leq 1
$$

for $\alpha=N$. This requices that $Q(\alpha) \geq 0$. where $Q(\alpha)$ is defined as in the assertion, and thus the assertion is proved.

We are now prepared to state and prove the theorem regarding a lower bound on the radii of convergence of the three series. 
Theorem 2 There exists $\mathcal{R}, 0<\mathcal{R}<\infty$, such that for $0<\tau<\mathcal{R}$, the series

$$
\sum_{\alpha=0}^{\infty} a_{\alpha} \tau^{\alpha-1} \cdot \sum_{\alpha=0}^{\infty} b_{\alpha} \tau^{\alpha-2} \text { and } \sum_{\alpha=0}^{\infty} c_{\alpha} \tau^{\alpha-2}
$$

converge absolutely for the calues of the system 's parameters given by (2.20).

Proof. Using Proposition 2 the theorem follows easily from the comparison test.

Considering the series for $\alpha \geq 5$ and using Proposition 2 we obtain the same radius of convergence as for the ordinary series

$$
R \geq \frac{1}{k}=\mathcal{R}_{1 b},
$$

where $\mathcal{R}_{16}$ denotes the estimated lower bound on the radii of convergence of the series.

\subsection{Psi Series}

In this section a series solution will be derived which will be valid if any of the three compatibility conditions (2.16), (2.18) and (2.19) are not met. As described in Section 1.3, in this case a general solution will be of the form

$$
u(t)=\sum_{k=0}^{\infty} \sum_{l=0}^{\infty} d_{k l} \tau^{k-p}\left(\tau^{k_{0}} \log \tau\right)^{l},
$$

where $p$ is the integer obtained from the leading order analysis and $k_{0}$ is the smallest resonance for which the compatibility condition is not met.

Fur purposes of further analysis, the doubly infinite series is now converted into a single series with polynomial coefficients. Rather than summing over all $k$ and $l$, Figure 4.14 illustrates that this is equivalent to summing over each line

$$
\left\{(k, l): k+k_{0} l=a\right\} \quad 0 \leq k, l<\infty,
$$

for every integer $a .0 \leq a<\infty$. Each line contains a finite number of points $(k, l)$ with non-negative integer coordinates. 
Letting $k+h_{0} l=\alpha,(2.26)$ bromes

$$
u(t)=\sum_{i x=0}^{\infty} \tau^{u-p} \sum_{k+k_{0} l=a x} d_{k l}(\log \tau)^{\prime} .
$$

Lastly, letting $z=\log (\tau)$ and $u_{i s}(z)=\sum_{k+k_{0} l=a r} l_{k l}(\log \tau)^{l}$, the solution becomess

$$
u(t)=\sum_{a=0}^{\infty} \tau^{u-p} w_{a x}(z) \text {. }
$$

Note that since $l=\frac{\alpha-k}{k_{0}}$, the order of $\omega_{a r}(z)$ is $\left\lfloor\frac{\alpha}{k_{0}}\right\rfloor$, where $\lfloor T\rfloor$ denotes the greatest integer less than or equal to $T$.

Using this resummation technique for the psi series solutions of the Lorenz sys. tem, we form the series solutions

$$
\begin{aligned}
& x(t)=\sum_{u=0}^{\infty} \tau^{u-1} f_{\alpha}(z), \\
& y(t)=\sum_{u=0}^{\infty} \tau^{u-2} g_{u x}(z), \\
& z(t)=\sum_{u=0}^{\infty} \tau^{u-2} h_{\alpha}(z),
\end{aligned}
$$

where

$$
\begin{aligned}
& f_{\alpha x}(z)=\sum_{k+k_{0} l=\alpha} \alpha_{k l l}(\log \tau)^{l}, \\
& y_{\alpha}(z)=\sum_{k+k_{j_{0} l=\alpha \alpha} b_{k l l}(\log \tau)^{l},} c_{k l}(\log \tau)^{l} .
\end{aligned}
$$

Differentiating these series with respect to time we obtain

$$
\begin{aligned}
& r^{\prime}(l)=\sum_{\alpha=0}^{x} \tau^{\prime n-2}\left(f_{\omega}^{\prime}(z)+(\alpha-1) f_{\alpha}(z)\right), \\
& y^{\prime}(t)=\sum_{a=0}^{\infty} \tau^{\alpha-3}\left(y_{1,}^{\prime}(z)+(\alpha-\underline{2}) g_{u x}(z)\right) \text {, } \\
& z^{\prime}(t)=\sum_{a=0}^{\infty} \tau^{u-3}\left(h_{1 \alpha}^{\prime}(z)+(\alpha-z) f_{d a}(z j)\right. \text {. }
\end{aligned}
$$

since it can easily be shown that the sum of the derivatives converges uniformly, and thus it is valid to differentiate term by term. Briefly, this is proved by showing 
that the sequence of partial sums is Caucly, and in fact uniformly Cauchy, and thus uniformly convergent.

Substituting (2.27) and (2.2S) into the Lorenz system (1.1) yields:

$$
\begin{aligned}
\sum_{\alpha=0}^{\infty}\left(\int_{\alpha}^{\prime}(z)+(\alpha-1) f_{\alpha}(z)+\sigma f_{\alpha-1}(z)-\sigma g_{\alpha}(z)\right) \tau^{\alpha-2} & =0, \\
\sum_{\alpha=0}^{\infty}\left(g_{\alpha}^{\prime}(z)+(\alpha-2) g_{\alpha}(z)-R f_{\alpha-2}(z)+\right. & \\
\left.g_{\alpha-1}+\sum_{j=0}^{\alpha} f_{\alpha-\beta}(z) h_{\beta}(z)\right) \tau^{\alpha-3} & =0, \\
\sum_{\alpha=0}^{\infty}\left(h_{\alpha}^{\prime}(z)+(\alpha-2) h_{\alpha}(z)+B h_{\alpha-1}(z)-\right. & \\
\left.\sum_{j=0}^{\alpha} f_{\alpha-\beta}(z) g_{\beta}(z)\right) \tau^{\alpha-3} & =0,
\end{aligned}
$$

after shifting the indices of summation to obtain identical powers of $\tau$ in each series. For these equations to be satisfied for all $t$ it is necessary that the coefficient $j$ each power of $\tau$ be zero. Thus we obtain the following three recursion relations for $f_{\alpha}(z)$, $g_{\alpha}(z)$ and $h_{\alpha}(z)$ :

$$
\begin{aligned}
f_{\alpha}^{\prime}(z)+(\alpha-1) f_{\alpha}(z)+\sigma f_{\alpha-1}(z)-\sigma g_{\alpha}(z) & =0, \\
g_{\alpha}^{\prime}(z)+(\alpha-2) g_{\alpha}(z)-R f_{\alpha-2}(z)+g_{\alpha-1}+\sum_{\beta=0}^{\alpha} f_{\alpha-\beta}(z) h_{\beta}(z) & =0, \\
h_{\alpha \alpha}^{\prime}(z)+(\alpha-2) h_{\alpha}(z)+B h_{\alpha-1}(z)-\sum_{\beta=0}^{\alpha} f_{\alpha-\beta}(z) g_{\beta}(z) & =0 .
\end{aligned}
$$

Substituting $\alpha=0$ into (2.29) yields

$$
\begin{aligned}
& f_{0}= \pm 2 i, \\
& y_{0}=\mp 2 i / \sigma, \\
& h_{0}=-2 / \sigma,
\end{aligned}
$$

or $f_{0}, g_{0}, h_{0}$ equal to 0 . These are equivalent to the $a_{0}, b_{0}$ and $c_{0}$ found for the Laurent series. In the case that $f_{0} . g_{0}$ and $h_{0}$ are all zero, $g_{1}$ and $h_{1}$ are also zero and all the polynomials $f_{\alpha}(z), y_{\alpha}(z)$ and $h_{\alpha}(z)$ become constant functions. In this case $f_{1}, g_{2}$ and $h_{2}$ are arlitrary. These are the initial conditions $x(0), y(0)$ and $z(0)$ 
respectively and $t_{0}$ is no longer a singularity. In other words, the psi series evolves as an ordinary series.

Substituting (2.30) into (2.29) yields

$$
\begin{aligned}
& f_{\alpha \alpha}^{\prime}(z)+(\alpha-1) f_{\alpha}(z)+\sigma f_{\alpha-1}(z)-\sigma g_{\alpha}(z)=0 . \\
& g_{\alpha}^{\prime}(z)+(\alpha-2) g_{\alpha}(z)-R f_{\alpha-2}(z)+I_{\alpha-1}+ \\
& +\sum_{\beta=1}^{\alpha-1} f_{\alpha-\beta}(z) h_{\beta}(z) \pm 2 i h_{\alpha}(z)-(2 / \sigma) f_{\alpha}(z)=0, \\
& h_{\alpha}^{\prime}(z)+(\alpha-2) h_{\alpha}(z)+B h_{\alpha-1}(z)- \\
& \sum_{\beta=1}^{\alpha-1} f_{\alpha-\alpha \beta}(z) g_{\beta}(z) \pm(2 i / \sigma) f_{\alpha}(z) \mp 2 i g_{\alpha}(z)=0,
\end{aligned}
$$

or equivalently in matrix form,

$$
\begin{aligned}
& \left(\begin{array}{l}
f_{\alpha}(z) \\
g_{\alpha}(z) \\
h_{\alpha}(z)
\end{array}\right)^{\prime}+\left(\begin{array}{ccc}
(\alpha-1) & -\sigma & 0 \\
-2 / \sigma & (\alpha-2) & \pm 2 i \\
\pm \frac{2 i}{\sigma} & \mp 2 i & (\alpha-2)
\end{array}\right)\left(\begin{array}{l}
f_{\alpha}(z) \\
y_{\alpha}(z) \\
h_{\alpha}(z)
\end{array}\right)
\end{aligned}
$$

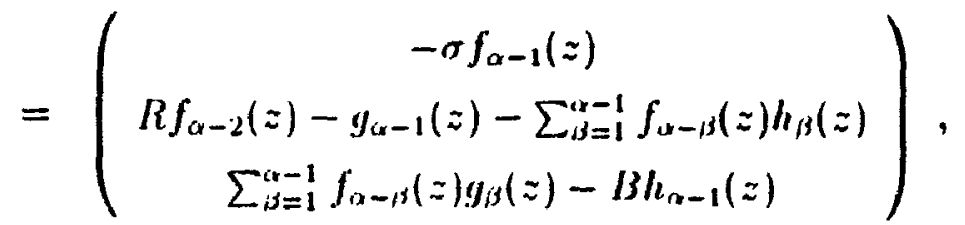

or

$$
v_{u}^{\prime}(z)+A_{u x} v_{a}(z)=V_{a}(z)=\left(\begin{array}{c}
V_{u 1} \\
V_{u z} \\
V_{u x 3}
\end{array}\right) \text {. }
$$

In order to prove the existence of a lower bound on the radii of convergence of the three series, we first establish a bound on the coeflicients of the series with the following proposition.

Proposition 3 There exists $K, 1<K<\infty$. such that for all $z<0$ and for all $\alpha \geq 5$,

$$
\left\|v_{u}(z)\right\| \leq \frac{(2 k-k z)^{u / k_{0}}}{\sqrt{\alpha+1}}
$$

where $k_{0}=2$ or $k_{0}=4$. 


\section{Proof.}

The proposition is a consequence of the following

Assertion 2 If.$V$ is such that the set of inequalities $Q$ are satisfied for $\alpha=N$. where $Q$ is defined by

$$
\begin{aligned}
& \alpha \geq 4+\frac{7 \sqrt{6}}{2 \sqrt{5}} P(\sigma) \sigma \\
& Q=\left\{\left(\frac{4}{49 P^{2}(\sigma)}\right) \alpha^{2}-\left(\frac{4}{i P(\sigma)}\left(\frac{8}{7 P(\sigma)}+\sqrt{\frac{3}{2}} J\right)-\pi^{2}\right) \alpha+\left(\left(\frac{8}{7 P(\sigma)}+\sqrt{\frac{3}{2}} J\right)^{2}-\pi^{2}\right) \geq 0\right\}, \\
& \left(\frac{4}{49 P^{2}(\sigma)}\right) \alpha^{2}-\left(\frac{4}{\pi P(\sigma)}\left(\frac{8}{7 P(\sigma !}+\sqrt{\frac{3}{2}} J J\right)-\pi^{2}\right) \alpha+\left(\left(\frac{8}{7 P(\sigma)}+\sqrt{\frac{3}{2}} J J\right)^{2}-\pi^{2}\right) \geq 0
\end{aligned}
$$

where

$$
\begin{gathered}
J=R+1+\sum_{\beta=1}^{k_{0}-1}\left(\left|h_{\beta}(z)\right|+\left|f_{\beta}(z)\right|\right), \\
J J=B+\sum_{3=1}^{k_{0}-1}\left(\left|f_{\beta}(z)\right|+\left|g_{\beta}(z)\right|\right)
\end{gathered}
$$

and

$$
\begin{aligned}
P(\sigma) & =\|P\|\left\|P^{-1}\right\| \\
& =\left\{\begin{array}{ll}
\frac{\pi}{3}\left(\frac{1}{\sigma}+1\right), & \sigma<\frac{7}{4} \\
\frac{4}{3}(\sigma+1), & \frac{7}{4}<\sigma<5 \\
\frac{8}{5} \sigma, & \sigma>5
\end{array},\right.
\end{aligned}
$$

and if

$$
\left\|v_{\alpha}(z)\right\| \leq \frac{(2 K-K z)^{\alpha / k_{0}}}{\sqrt{\alpha+1}}
$$

for all $a<N$, then

$$
\left\|v_{\alpha}(z)\right\| \leq \frac{(2 K-K z)^{\alpha / k_{0}}}{\sqrt{\alpha+1}}
$$

for $\alpha=N$.

Given an $N$. since $f_{\alpha}(z), g_{\alpha}(z)$, and $h_{\alpha}(z)$ are polynomials of order $\left\lfloor\frac{\alpha}{k_{0}}\right\rfloor$, it is possible to find a $K$ such that (2.34) holds for $5 \leq \alpha \leq N-1$. How such a $K$ is calculated is shown in the next section.

The proposition will then follow by induction. if $N$ is chosen such that the set of inequalities $Q$ loold for all $a \geq N$. 
We now prove the claim.

We begin by solving (2.33) for $v_{\alpha}$.

Multiplying (2.33) through by the integrating factor $e^{\text {:tuz }}$ yields

$$
e^{A_{\alpha} z} \mathrm{~V}_{\alpha}(z)^{\prime}+A_{\alpha} e^{A_{\alpha} z} \mathrm{~V}_{\alpha}(z)=\mathrm{V}_{\alpha}(z) e^{A_{\alpha} z}
$$

i.e.,

$$
\left(e^{A_{\alpha} z} V_{\alpha}(z)\right)^{\prime}=e^{A_{\alpha} z} V_{\alpha}(z)
$$

Integrating both sides from $-\infty$ to $z$. we obtain

$$
\left.e^{A_{\alpha} q} \mathrm{v}_{\alpha}(q)\right|_{-\infty} ^{z}=\int_{-\infty}^{z} e^{A_{\alpha}:} \mathrm{V}_{\alpha}(q) d q
$$

Since $\lim _{q \rightarrow-\infty} e^{A_{\alpha} q} \mathbf{v}_{\alpha}(z)=0$, after multiplying through by $e^{-A_{\alpha} z}$, we obtain

$$
\mathbf{V}_{\alpha}(z)=\int_{-\infty}^{b} e^{A_{\alpha}(q-z)} \mathbf{V}_{\alpha}(q) d q
$$

Noting that $e^{T_{a}(y-z)}=P e^{D_{a}(q-z)} P^{-1}(2.39)$ becomes

$$
\mathrm{V}_{\alpha}(z)=\int_{-\infty \infty}^{z} P e^{D_{\alpha}(q-z)} P^{-1} \mathbf{V}_{\alpha}(q) d q
$$

where $P$, the matrix of eigenvectors of $A_{\alpha}$, is given by

$$
P=\left(\begin{array}{ccc} 
\pm \sigma / 2 & \mp \sigma & \pm \sigma / 2 \\
\mp 1 & \mp 1 & \pm 3 / 2 \\
i & i & i
\end{array}\right)
$$

and

$$
D_{\alpha}=P^{-1} A_{\alpha} P=\left(\begin{array}{ccc}
\alpha+1 & 0 & 0 \\
0 & \alpha-2 & 0 \\
0 & 0 & \alpha-4
\end{array}\right) .
$$

To estimate tije norm of $v_{\alpha}(z)$ we must estimate each of the norms within the integral. Since $q \leq z \Rightarrow q-z \leq 0$, and $\alpha-4<\alpha-2<\alpha-1$, we obtain

$$
\begin{aligned}
\left\|e^{D_{m}(q-z)}\right\| & =\max \left(e^{(\alpha+1)(q-z)}, e^{(\alpha-z)(y-z)}, e^{(\alpha-4)(y-z)}\right) \\
& =e^{(\alpha-4)(q-z)} .
\end{aligned}
$$


Letting

$$
\begin{aligned}
P(\sigma) & =\|P\|\left\|P^{-1}\right\| \\
& = \begin{cases}\frac{7}{3}\left(\frac{1}{\sigma}+1\right), & \sigma<\frac{7}{3} \\
\frac{4}{3}(\sigma+1), & \frac{7}{7}<\sigma<5 \\
\frac{8}{5} \sigma, & \sigma>5\end{cases}
\end{aligned}
$$

and substituting (2.41) and (2.42) into (2.40) to estimate $\left\|v_{a}(z)\right\|$ we obtain

$$
\left\|\mathrm{v}_{\alpha}(z)\right\| \leq P(\sigma) \int_{-\infty}^{z} e^{(\alpha-4)(q-z)}\left\|\mathrm{V}_{\alpha}(q)\right\| d q
$$

Given that (2.34) holds for $5 \leq \alpha \leq N-1$, for $\alpha=N$

$$
\begin{aligned}
& \left|V_{\alpha 1}(z)\right|=\sigma\left|f_{\alpha-1}(z)\right| \\
& \leq \sigma \frac{(2 K-K z)^{\alpha / k_{0}}}{\sqrt{\alpha}} \\
& \left|\mathbf{V}_{\alpha 2}(z)\right|=R\left|f_{\alpha-2}(z)\right|+\left|g_{\alpha-1}(z)\right|+\sum_{\beta=k_{0}}^{\alpha-k_{0}}\left|h_{\beta}(z)\right|\left|f_{\alpha-\beta}(z)\right| \\
& +\sum_{\beta=1}^{k_{0}-1}\left|h_{\beta}(z)\right|\left|f_{\alpha-\beta}(z)\right|+\sum_{\beta=\alpha-k_{0}+1}^{\alpha-1}\left|h_{\beta}(z)\right|\left|f_{\alpha-\beta}(z)\right| \\
& \leq R \frac{(2 K-K z)^{\frac{\alpha-2}{k_{0}}}}{\sqrt{\alpha-1}} \\
& \left.+\left(1+\sum_{\beta=1}^{k_{0}-1} i\left|h_{\beta}(z)\right|+\left|f_{\beta}(z)\right|\right)\right) \frac{(2 K-K z)^{\alpha-1 / k_{0}}}{\sqrt{\alpha}} \\
& +\sum_{\beta=2}^{\alpha-2} \frac{(2 K-K z)^{\alpha / k_{0}}}{\sqrt{\beta+1} \sqrt{\alpha-\beta}} \\
& \leq\left(\frac{R+1+\sum_{\beta=1}^{k_{0}-1}\left(\left|h_{\beta}(z)\right|+\left|f_{\beta}(z)\right|\right)}{\sqrt{\alpha-1}}+\pi\right)(2 K-K z)^{\alpha / k_{0}} \text {, }
\end{aligned}
$$

using the fact that

$$
\lim _{\alpha \rightarrow \infty} \sum_{\beta=0}^{\alpha-1} \frac{1}{\sqrt{\beta+1}} \frac{1}{\sqrt{\alpha-\beta}}=\pi
$$


Similarly,

$$
\begin{aligned}
\left|\mathbf{V}_{\alpha 3}(z)\right|= & B\left|h_{\alpha-1}(z)\right|+\sum_{\beta=k_{0}}^{\alpha-k_{0}}\left|g_{\beta}(z)\right|\left|f_{\alpha-\beta}(z)\right|+\sum_{\beta=1}^{k_{0}-1}\left|g_{\beta}(z)\right|\left|f_{\alpha-\beta}(z)\right| \\
& +\sum_{\beta=\alpha-k_{0}+1}^{\alpha-1}\left|g_{\beta}(z)\right|\left|f_{\alpha-\beta}(z)\right|, \\
\leq & \left(\frac{B+\sum_{\beta=1}^{k_{0}-1}\left(\left|g_{\beta}(z)\right|+\left|f_{\beta}(z)\right|\right)}{\sqrt{\alpha-1}}+\pi\right)\left(2 K-K^{\prime} z\right)^{\alpha / k_{0}} .
\end{aligned}
$$

Letting

$$
G_{\alpha}=\left(\begin{array}{c}
\frac{a}{\sqrt{\alpha}} \\
\frac{R+1+\sum_{\beta=1}^{k_{0}-1}\left(\left|h_{\beta}(z)\right|+\left|f_{\beta}(z)\right|\right)}{\sqrt{\alpha-1}}+\pi \\
\frac{B+\sum_{\beta=1}^{k_{0}-1}\left(\left|g_{\beta}(z)\right|+\left|f_{\beta}(z)\right|\right)}{\sqrt{\alpha-1}}+\pi
\end{array}\right)=\left(\begin{array}{c}
G_{\alpha 1} \\
G_{\alpha 2} \\
G_{\alpha 3}
\end{array}\right)
$$

we obtain

$$
\left\|\mathbf{V}_{\alpha}(z)\right\| \leq\left\|G_{\alpha}\right\|(2 K-K z)^{\alpha / k_{0}}
$$

and (2.43) becomes

$$
\left\|\mathrm{v}_{\alpha}(z)\right\| \leq P(\sigma)\left\|G_{\alpha}\right\| \int_{-\infty}^{z} e^{(\alpha-4)(q-z)}(2 K-K q)^{\alpha / k_{0}} d q
$$

To estimate the R.H.S. we need the following lemma.

Lemma 1

$$
\int_{-\infty}^{z} e^{(\alpha-4)(q-z)}(2 K-K q)^{\alpha / k_{0}} d q \leq \frac{7(2 K-K z)^{\alpha / k_{0}}}{2(\alpha-4)}
$$

for $\alpha>4$

Proof. Integrating the L.H.S. of (2.46) by parts yields

$$
\begin{aligned}
& \int_{-\infty}^{z} e^{(\alpha-4)(q-z)}(2 K-K q)^{\alpha / k_{0}} d q \\
& =\left.\frac{e^{(\alpha-4)(q-z)}}{(\alpha-4)}(2 K-K z)^{\alpha / k_{0}}\right|_{-\infty} ^{z}-\frac{1}{(\alpha-4)} \int_{-\infty}^{z} e^{(\alpha-4)(q-z)} \frac{\alpha}{k_{0}}(-K)(2 K-K q)^{\frac{z}{k_{0}}-1} d q \\
& =\frac{(2 K-K z)^{\alpha / k_{0}}}{(\alpha-4)}-\frac{\frac{\alpha}{k_{0}}(-K)}{(\alpha-4)} \int_{-\infty}^{z} e^{(\alpha-4)(q-z)} \frac{\alpha}{k_{0}}(-K)(2 K-K q)^{\frac{\alpha}{k_{0}}-1} \\
& \quad \text { for } \alpha>4 .
\end{aligned}
$$


After $m+1$ integrations by parts, we obtain

$$
\begin{aligned}
& \int_{-\infty}^{z} e^{(\alpha-4)(q-z)}(2 K-K q)^{\alpha / k_{0}} d q \\
& =\frac{(2 K-K z)^{\alpha / k_{0}}}{(\alpha-4)}-\frac{\frac{\alpha}{k_{0}}(-K)}{(\alpha-4)^{2}}(2 K-K z)^{\frac{\alpha}{k_{0}}-1} \\
& +\frac{\frac{\alpha}{k_{0}}\left(\frac{\alpha}{k_{0}}-1\right)\left(-K^{\prime}\right)^{2}}{(\alpha-4)^{3}}(2 K-K z)^{\frac{\alpha}{k_{0}}-2}-\cdots \\
& +\frac{(-1)^{m} \frac{a}{k_{0}}\left(\frac{\alpha}{k_{0}}-1\right) \cdots\left(\frac{\alpha}{k_{0}}-m\right)(-K)^{m+1}}{(\alpha-4)^{m+1}} \int_{-\infty}^{z} e^{(\alpha-4)(q-z)}\left(2 K-K^{\prime} q\right)^{\frac{\alpha}{k_{0}}-m-1} d q .
\end{aligned}
$$

To simplify we let

$$
m=\left\lfloor\frac{a}{k_{0}}\right\rfloor, \quad \gamma=\frac{\alpha}{k_{0}} \text { and } \quad X=(2 K-K z)
$$

Then

$$
\begin{aligned}
& \int_{-\infty}^{z} e^{(\alpha-4)(q-z)}(2 K-K q)^{\alpha / k_{0}} d q \\
& =\frac{X^{\gamma}}{(\alpha-4 j}+\frac{\gamma K X^{\gamma-1}}{(\alpha-4)^{2}}+\cdots \\
& +\frac{\gamma(\gamma-1) \cdots(\gamma-m) K^{m+1}}{(\alpha-4)^{m+1}} \int_{-\infty}^{z} e^{(\alpha-4)(q-z)}\left(2 K-K^{\prime} q\right)^{\frac{\alpha}{k_{0}}-m-1} d q .
\end{aligned}
$$

Let us estimate the last integral. Since $\gamma-m-1<0$, for $q \leq z$

$$
(2 K-K q)^{\gamma-m-1} \leq X^{\gamma-m-1}
$$

and

$$
\begin{aligned}
& \int_{-\infty}^{z} c^{(\alpha-4)(q-z)}(2 K-K q)^{\frac{\alpha}{2}-m-1} d q \\
& \leq X^{\gamma-m-1} \int_{-\infty}^{z} e^{(\alpha-4)(q-z)} d q \\
& =\frac{.^{\gamma-m-1}}{a-4} .
\end{aligned}
$$

Thus

$$
\begin{gathered}
\int_{-\infty}^{z} e^{(\alpha-4)(q-z)}(2 K-K q)^{\alpha / k_{0}} d q \\
\leq \frac{X^{\gamma}}{(\alpha-4)}+\frac{\gamma K X^{\gamma-1}}{(\alpha-4)^{2}}+\cdots
\end{gathered}
$$




$$
\begin{aligned}
& +\frac{\gamma(\gamma-1) \cdots(\gamma-m) K^{m+1}}{(\alpha-4)^{m+1}} K^{m+1} X^{\gamma-m-1} \\
& =\frac{X^{\gamma}}{(\alpha-4)}\left(1+\frac{\gamma}{(\alpha-1)}\left(\frac{K}{X}\right)+\frac{\gamma(\gamma-1)}{(\alpha-4)^{2}}\left(\frac{h}{X}\right)^{2}+\cdots\right. \\
& \left.+\frac{\gamma(\gamma-1) \cdots(\gamma-m)}{(\alpha-1)^{m+1}}\left(\frac{K}{X}\right)^{m+1}\right) .
\end{aligned}
$$

Since for $z<0$

$$
\frac{K}{X}=\frac{K}{2 K-K z}=\frac{1}{2-z} \leq \frac{1}{2}
$$

and for $j \geq 2$

$$
\frac{\gamma-j}{a-4} \leq \frac{1}{2}
$$

we obtain

$$
\begin{aligned}
& \int_{-\infty}^{z} e^{(\alpha-4)(q-z)}\left(2 K-K^{\prime} q\right)^{\alpha / k_{0}} d q \\
& \leq \frac{X^{\gamma}}{(\alpha-4)}\left(1+\frac{\gamma}{2(\alpha-4)}\left(1+\frac{\gamma-1}{2(\alpha-4)}\left(1+\frac{1}{4}+\left(\frac{1}{4}\right)^{2}+\cdots+\left(\frac{1}{4}\right)^{m-1}\right)\right)\right) \\
& \leq \frac{X^{\gamma}}{(\alpha-4)}\left(1+\frac{\gamma}{2(\alpha-4)}\left(1+\frac{\gamma-1}{2(\alpha-4)}\left(\frac{4}{3}\right)\right)\right) \\
& \leq \frac{7 X^{\gamma}}{2(\alpha-4)}
\end{aligned}
$$

which concludes the proof of Lemma 1.

We can now employ the estimate found in Lemma 1 in (2.45) to obtain

$$
\left\|v_{\alpha}(z)\right\| \leq P(\sigma)\left\|C_{\alpha}\right\| \frac{7(2 K-\kappa z)^{\alpha / k_{\alpha}}}{2(\alpha-4)} .
$$

Recall that we are attempting to show that

$$
\left\|\mathrm{v}_{\alpha}(z)\right\|<\frac{(2 K-K z)^{\alpha / k_{0}}}{\sqrt{\alpha+1}},
$$

for $\alpha=N$. This requires that the inequality

$$
P(\sigma)\left\|C_{i, x}\right\| \frac{T}{2(\alpha-4)} \leq \frac{1}{\sqrt{\alpha+1}}
$$

hold for $\alpha=N$. 
This condition will be examined for the three cases $\left\|G_{\alpha}\right\|=G_{\alpha 1}, G_{\alpha 2}$ and $G_{\alpha 3}$.

When $\left\|G_{\alpha}\right\|=G_{\alpha 1},(2.48)$ becomes

$$
P(\sigma) \frac{\sigma}{\sqrt{\alpha}} \frac{7}{2(\alpha-4)} \leq \frac{1}{\sqrt{\alpha+1}} .
$$

Since $\frac{\sqrt{\alpha+1}}{\sqrt{a}} \leq \sqrt{\frac{6}{5}}$, this is equivalent to

$$
\alpha \geq 4+\frac{7 \sqrt{6}}{2 \sqrt{5}} P(\sigma) \sigma
$$

which can always be satisfied by sufficiently large $N$.

If we let

$$
J=\left(R+1+\sum_{\beta=1}^{k_{0}-1}\left(\left|h_{\beta}(z)\right|+\left|f_{\beta}(z)\right|\right)\right)
$$

if $\left\|G_{\alpha}\right\|=G_{\alpha 2}$, and let

$$
J=\left(B+\sum_{\beta=1}^{k_{0}-1}\left(\left|f_{\beta}(z)\right|+\left|g_{\beta}(z)\right|\right)\right)
$$

if $\left\|G_{\alpha}\right\|=G_{\alpha 3}$, for these two cases (2.48) becomes

$$
P(\sigma)\left(\frac{J}{\sqrt{\alpha-1}}+\pi\right) \frac{7}{2(\alpha-4)} \leq \frac{1}{\sqrt{\alpha+1}} .
$$

Since $\frac{\sqrt{\alpha+1}}{\sqrt{u-1}} \leq \sqrt{\frac{3}{2}}$, this is equivalent to

$$
\pi \sqrt{\alpha+1} \leq \frac{2}{7 P(\sigma)} \alpha-\left(\frac{8}{7 P(\sigma)}+\sqrt{\frac{3}{2}} J\right) .
$$

Squaring both sides and moving all terms to the R.H.S. gives the equivalent condition

$$
\left(\frac{4}{49 P^{2}(\sigma)}\right) \alpha^{2}-\left(\frac{4}{i P(\sigma)}\left(\frac{8}{i P(\sigma)}+\sqrt{\frac{3}{2}} J\right)-\pi^{2}\right) \alpha+\left(\left(\frac{8}{7 P(\sigma)}+\sqrt{\frac{3}{2}} J\right)^{2}-\pi^{2}\right) \geq 0 \text {. }
$$

We can now see that if $N$ satisfies $Q(2.35)$ the assertion will be proved, and thus Proposition 3 as well.

We are now prepared to state and prove the theorem regarding a lower bound on the radii of convergence of the three series. 
Theorem 3 There exists $R, 0<R<\infty$, such that for $0<\tau<\mathcal{R}$, the psi series

$$
\sum_{\alpha=0}^{\infty} f_{\alpha}(z) \tau^{\alpha-1}, \sum_{\alpha=0}^{\infty} y_{\alpha}(z) \tau^{\alpha-2} \text { and } \sum_{\alpha=0}^{\infty} h_{\alpha}(z) \tau^{\alpha-2}
$$

converge absolutely for all values of the system is parameters $\sigma, B$ and $R$.

Using Proposition 3 the theorem follows from the comparison test.

Considering the series for $\alpha \geq 5$ and using the claim (2.34) we have

$$
\sum_{a=5}^{\infty} \mathbf{v}_{\alpha}(z) \tau^{u-a} \leq \sum_{\alpha=5}^{\infty}(2 K-K z)^{\alpha / k_{0}} \tau^{u-a}
$$

where $a=1$ or $a=2$.

Applying the ratio test to this series yields

$$
\lim _{\alpha \rightarrow \infty}\left(\frac{\sqrt{\alpha+1}}{\sqrt{\alpha+2}}(2 K-K z)^{1 / k_{0}} \tau\right)=\tau(2 K-K z)^{1 / k_{0}}
$$

Therefore the three psi series converge iff

$$
\tau(2 K-K z)^{1 / k_{0}}<1
$$

Substituting $\tau=e^{z}$, the condition for convergence becornes

$$
K(2-z)<e^{-k_{0} z}
$$

and letting $s=-z>0$, becomes

$$
h(2+s)<e^{k_{0} s}
$$

Let $s_{0}$ denote the point of intersection of the line $y=K s+2 K$ and the curve $y=e^{k_{0} s}$. From Figure 4.15, one can see tiat for all $s>s_{0}, K(2+s)<e^{k_{0} 0}$. Thus for all $z<z_{0}$, where $z_{0}=-s_{0}$, or equivaiently for all $\tau, 0<\tau<e^{z_{0}}$, the psi series will converge. This completes the proof of the theorem. 


\subsubsection{Plots and Discussion}

In this section, for specific parameter values the estimated lower bound on the radii of convergence $\left(\mathcal{R}_{l b}\right)$ will be calculated.

The number $K$ must first be calculated.

Let

$$
L_{\alpha}(z)=\left(\frac{\left\|v_{\alpha}(z)\right\|}{(2-z)^{\alpha / k_{0}}}\right)^{k_{0} / \alpha}(\alpha+1)^{1 / \alpha} .
$$

Since $f_{a}(z)$ is a polynomial in $z$ of order $\left\lfloor\alpha / k_{0}\right\rfloor$,

$$
\begin{aligned}
\left|f_{\alpha}(z)\right| & =\left|\sum_{n=0}^{\left.\mid \alpha / k_{0}\right\rfloor} a_{n} z^{n}\right| \\
& \leq \sum_{n=0}^{\left\lfloor\alpha / k_{0}\right\rfloor}\left|a_{n}\right|\left|z^{n}\right| \\
& \leq \sum_{n=0}^{\left\lfloor\alpha / k_{0}\right\rfloor}\left|a_{n}\right|(2-z)^{n} \\
& \leq\left(\sum_{n=0}^{\left[\alpha / k_{0}\right\rfloor}\left|a_{n}\right|\right)(2-z)^{\alpha / k_{0}} .
\end{aligned}
$$

Thus

$$
\left\|v_{\alpha}\right\| \leq\left(\sum_{n=0}^{\left\lfloor\alpha / k_{0}\right\rfloor}\left|d_{n}\right|\right)(2-z)^{\alpha / k_{0}},
$$

where $d_{n}$ denotes the coefficients belonging the series $f_{\alpha}(z), g_{\alpha}(z)$ or $h_{\alpha}(z)$ for which the sum of the absolute values of the coefficients is the largest.

Substituting this estimate into the formula for $L_{a}(z)$ we obtain

$$
L_{\alpha}(z) \leq\left(\sum_{n=0}^{\left.\mid \alpha / k_{0}\right\rfloor}\left|d_{n}\right|\right)^{\alpha / k_{0}}(\alpha+1)^{1 / \alpha} .
$$

Therefore it suffices to have

$$
\kappa=\max _{k_{0} \leq \alpha \leq N-1} L_{\alpha}
$$

The estimate $\mathcal{R}_{t b}$ is now computed. The arbitrary singularity is placed at $t=0$ and the two arbitrary coefficients are given the valus zero. The parameter values 
Table 2.1: $\boldsymbol{R}_{t b}$ and $\mathcal{R}_{n c t}$ as $R$ varies, for both the $k_{0}=2$ and $k_{0}=4$ cases

\begin{tabular}{|c|c|c|c|c|c|c|}
\hline \multirow[b]{2}{*}{$\boldsymbol{R}$} & \multicolumn{3}{|c|}{$k_{0}=2$} & \multicolumn{3}{|c|}{$k_{0}=4$} \\
\hline & $\kappa$ & $\mathcal{R}_{1 b}$ & $\mathcal{R}_{a c t}$ & $\kappa$ & $\mathcal{R}_{1 b}$ & $\mathcal{R}_{\text {act }}$ \\
\hline .50 & 6.93 & .0400 & $.075-.08$ & 2.21 & .1849 & $1.40-1.50$ \\
\hline 1.50 & 6.46 & .0432 & $.075-.08$ & 3.85 & .1011 & $0.80-0.90$ \\
\hline $14.2 \mathrm{~J}$ & 34.56 & .0063 & $.075-.08$ & 25.03 & .0129 & $0.40-0.42$ \\
\hline 24.74 & 54.65 & .0038 & $.090-.10$ & 39.51 & .0079 & 0.35-0.3 \\
\hline 100.00 & 165.75 & .0011 & $.045-.05$ & 120.88 & .0024 & $0.15-0.17$ \\
\hline
\end{tabular}

$\sigma=10, B=\frac{8}{3}, R=28$ are chosen as for the ordinary series. The derivation of the formula for $f_{\alpha}(z), g_{\alpha}(z)$ and $h_{\alpha}(z)$ can be found in Appendix A.

The maxiraum of $L(\alpha)$ occurs at $\alpha=5$, as shown in Figure 4.16. $\mathcal{R}_{\text {est }}$ is approximately 0.02 and the actual radius of the series $\left(\mathcal{R}_{\text {act }}\right)$ estimated by thr ratio test, for these initial conditions is approxinately 0.09 .

Values of $\mathcal{R}_{b b}$ and $\mathcal{R}_{a c t}$ obtained for the $k_{0}=2$ and $k_{0}=4$ cases, as the system parameter $R$ is varied, are compared in Table 2.1. $B=\frac{8}{3}$ and $\sigma=\frac{4}{3}$ are chosen to satisfy the compatibility condition for the resonance at 2 , making $k_{0}=4$. The values $B=\frac{8}{3}$ and $\sigma=\frac{5}{3}$ do not satisfy any of the compatibility conditions, making $k_{0}=2$.

For the governing equations of anharmonic oscillators, researchers have found that the higher the order at which the logarithmic terms enter (i.e. the higher the $k_{0}$ ), the smaller the chaotic region is [10]. This analysis is interesting in view of their findings.

Lastly, the truncation error as a function of time is given by

$$
E_{n}=\left((2 \kappa-K \log \tau)^{\frac{1}{\hat{\alpha}_{0}} \tau}\right)^{n+1}
$$




\section{Chapter 3}

\section{The Duffing Equation}

The foregoing analysis for the Lorenz equations is now repeated for the Duffing equation, of which there are many forms. Like the Lorenz equations it is a well studied system. Detailed dynamical studies have been completed by many researchers, most extensively Ueda [67]. The form of the equation used by Ueda is chosen for the ordinary series analysis. Painlevé analysis has been undertaken by many researchers [30]. The various forms of the equation give rise to different types of psi series. The parameter values which allow logarithmic singularities are chosen in order to parallel the Lorenz analysis.

\subsection{Ordinary Series}

First the recursion relation for the ordinary series is obtained. Substituting

$$
x(t)=\sum_{\alpha=0}^{\infty} a_{\alpha} \tau^{\alpha},
$$

where $\tau=\left(t-t_{1}\right)$ and $t_{1}$ is an ordinary point, and $F(t)$ as a power series about $t_{1}$ into Duffing s equation (1.2), we obtain

$$
\begin{gathered}
\sum_{\alpha=0}^{\infty} a_{\alpha}(\alpha)(\alpha-1) \tau^{\alpha-2}+k \cdot \sum_{\alpha=0}^{\infty} a_{\alpha}(\alpha) \tau^{\alpha-1}+p \sum_{\alpha=0}^{\infty} \sum_{\beta=0}^{\alpha} \sum_{\gamma=0}^{\beta} a_{\gamma} a_{\beta-\gamma} a_{\alpha-\beta} \tau^{\alpha}= \\
B_{t} \sum_{\alpha=0}^{\infty} \frac{F^{(\alpha)}\left(t_{1}\right)}{\alpha !} \tau^{\alpha u}+B_{0},
\end{gathered}
$$

where $F^{(\alpha)}\left(t_{1}\right)$ denotes the $\alpha^{\text {th }}$ derivative of $F(t)$, evaluated at $t_{1}$. 
Equating coelficients of equal powers of $\tau$ gives the recursion relation

$$
a_{\alpha}=\frac{1}{\alpha(\alpha-1)}\left[-k(\alpha-1) a_{\alpha-1}-p\left(\sum_{\beta=0}^{\alpha-2} \sum_{\gamma=0}^{3} a_{\gamma} a_{\beta-\gamma} a_{\alpha-\beta-2}\right)+B_{1} \frac{F\left(t_{1}\right)^{(\alpha-2)}}{(\alpha-2) !}+B_{0}\right]
$$

In order to prove the existence of a lower bound on the radii of convergence of the series, we first establish a bound on the coefficients of the serics with the following proposition.

Proposition 4 For all $M, 0<M<1$, there exists $K$. $\frac{1}{M}<K<\infty$, such that for all $\alpha \geq \mathrm{I}$

$$
\left|u_{\alpha}\right| \leq M K^{\alpha} \text {. }
$$

\section{Proof.}

The proposition is a consequence of the following Assertion 3 If $N$ is such for $\alpha=N$,

$$
\begin{aligned}
& \alpha^{2}\left(1-\frac{M^{2}}{2}\right)-\alpha\left(1+k-\frac{7 M^{2}}{2}\right) \\
& -\left(6 M M^{2}+3 p\left|a_{0}\right| M+3 p\left|a_{0}\right|^{2}+A B_{1}+B_{0}-k\right) \geq 0 .
\end{aligned}
$$

and if

$$
\left|a_{a r}\right| \leq M K^{a}
$$

for all $\alpha<N$, then

$$
\left|a_{\alpha}\right| \leq M K^{\prime a}
$$

for $\alpha=N$.

The proposition will then follow by induction if $N$ is chosen such that the inequality (3.2) holds for all $\alpha \geq N$, and $k^{\prime}$ is defined by

$$
K=\max _{1 \leq \alpha \leq N-1} L(\alpha)
$$

where

$$
L(\alpha)=\left(\frac{\left|a_{c}\right|}{. I}\right)^{1 / \alpha}
$$


We now prove the assertion.

Given that (3.1) holds for $1 \leq \alpha \leq N-1$, for $\alpha=N$ we obtain

$$
\begin{aligned}
& \left|a_{\alpha}\right| \leq \frac{1}{\alpha(\alpha-1)}\left[k(\alpha-1)\left|a_{\alpha-1}\right|+p\left(\sum_{\beta=0}^{\alpha-2} \sum_{\gamma=0}^{\beta}\left|a_{\gamma}\right|\left|a_{\beta-\gamma}\right|\left|a_{\alpha-\beta-2}\right|\right)+\right. \\
& \left.B_{1} \frac{\left|F\left(t_{1}\right)^{(\alpha-2)}\right|}{(\alpha-2) !}+B_{0}\right] \text {, } \\
& \leq \frac{1}{\alpha(\alpha-1)}\left(k(\alpha-1) M K^{\alpha-1}+M^{3} p \sum_{\beta=2}^{\alpha-3} \sum_{\gamma=1}^{\beta-1} K^{\gamma \gamma} K^{\gamma \beta-\gamma} K^{\alpha-\beta-2}\right. \\
& \left.+3 p M^{2} \sum_{\beta=1}^{\alpha-3}\left|a_{0}\right| K^{-\beta} K^{\alpha-j-2}+3 p\left|a_{0}\right|^{2} M I K^{\alpha-2}+A B_{1}+B_{0}\right), \\
& \leq \frac{1}{a(\alpha-1)}\left(k(\alpha-1)+M^{2}\left(\frac{\alpha^{2}-7 \alpha+12}{2}\right)\right. \\
& \left.+3 p\left|a_{0}\right| M+3 p\left|a_{0}\right|^{2}+A B_{1}+B_{0}\right) M K^{\alpha a} \text {, }
\end{aligned}
$$

where $A$ is the magnitude of the forcing term $F(t)$.

Therefore, for (3.1) to hold for $\alpha=N$, it is necessary that the coefficient of $M K^{\alpha}$ be less than or cciual to one. Thus the following inequality must hold for $\alpha=N$ :

$$
\begin{aligned}
& \alpha^{2}\left(1-\frac{M^{2}}{2}\right)-\alpha\left(1+k-\frac{\pi M^{2}}{2}\right) \\
& -\left(6 M^{2}+3 \mu\left|a_{0}\right| M+3 p\left|a_{0}\right|^{2}+A B_{1}+B_{0}-k\right) \geq 0 .
\end{aligned}
$$

Thus the assertion and proposition are proved.

We are now prepared to state and prove the theorem regarding a lower bound on the radius of convergence of the series.

Theorem 4 There exists $\mathcal{R} .0<\mathcal{R}<\infty$. such that for $0<|\tau|<\mathcal{R}$, the series

$$
\sum_{\alpha=0}^{\infty} u_{\alpha} t^{\alpha}
$$

converges absolutely for all values of the system's parameters, $B_{0}, B_{1}$ and $k$. 
Proof. Using Proposition the theorem follows easily from the comparison test.

Considering the series for $\alpha \geq 1$ we have

$$
\sum_{\alpha=1}^{\infty} a_{\alpha} \tau^{\alpha} \leq\left\|\sum_{\alpha=1}^{\infty} a_{\alpha} \tau^{\alpha}\right\| \leq \sum_{\alpha=1}^{\infty}\left|u_{\alpha} \| \tau\right|^{\alpha} \leq \sum_{\alpha=1}^{\infty}\left(K^{\alpha+}+B\right)|r|^{\alpha *} .
$$

Applying the ratio test to this last series gives

$$
\lim _{\alpha \rightarrow \infty}\left[\frac{\left(K^{\alpha+1}+B\right)}{\left(K^{\alpha}+B\right)}|\tau|\right]=K|\tau| \text {. }
$$

and thereiore

$$
\mathcal{R} \geq \frac{1}{\kappa}=\boldsymbol{R}_{i b},
$$

where $\mathcal{R}_{l b}$ denotes the estimated lower bound on the radii of convergence of the series.

\subsubsection{Plots and Discussion}

Choosing the values $p=1, B_{0}=0.03, B_{1}=0.16$ and $k=0.05$, to parallel the research done by Ueda [67], $\mathcal{R}_{b b}$ is calculated and the solution plotted by repeatedly re-expanding the series about a point within the radius of convergence.

The actual radius of the series $\left(\mathcal{R}_{\text {nct }}\right)$ for the initial conditions $(0,0)$, as determined by Hadamard's formula, is approximately 1 . Since $\left|a_{a}\right|$ is bounded by $B=0.26$ for all $\alpha$, the radius takes on its maximum possible value of one.

Plots of the trajectory defined by $x(t)$ and of the attractor are shown in Figure 4.18 and Figure 4.19. The initial conditions are $\left(x(0), x^{\prime}(0)\right)=(0,0)$ and the series is truncated to twenty-five terms. Figure 4.20 and Figure 4.21 show the corresponding plots obtained by numerical integration for the same initial conditions and parameter values, which are identical to those obtained with the series solutions.

Figure 4.22 illustrates the chaotic nature of the Duffing equation. Two trajectories with initial conditions differring by 0.01 diverge after a finite time.

As $B_{1}, B_{0}$ and $k$ are increased, both $\mathcal{R}_{l b}$ and $\mathcal{R}_{a c t}$ are found to decrease. This supports the findings of other researchers that as the forcing is increased, the singularities condense $[5,7,52,55]$. 


\subsection{Laurent Series}

Unless $B_{1}$ is zero, the Laurent series will not be valid for any parameter values, since the cumpatibility condition requires that the derivative of the forcing term, evaluated at the arbitrary singularity, take on a specific value, rendering the singularity no longer arbitrary.

\subsection{Psi Series}

In this section the psi series solution is formulated as for the Lorenz equations.

The systern (1.2) is first converted ':to two first-order equations

$$
\begin{gathered}
x^{\prime}=y, \\
y^{\prime}=-k y-p x^{3}+B_{1} F(t)+B_{0} .
\end{gathered}
$$

Solutions $x(t)$ and $y(t)$ of the form

$$
\begin{aligned}
& x(t)=\sum_{\alpha=0}^{\infty} \tau^{\alpha-1} f(z), \\
& y(t)=\sum_{u=0}^{\infty} \tau^{\alpha-2} g_{\alpha}(z),
\end{aligned}
$$

where

$$
\begin{aligned}
& f_{\alpha}(z)=\sum_{i+k_{0} l=\alpha} a_{k l}(\log \tau)^{i}, \\
& g_{. l}(z)=\sum_{k:-k_{0} l=\alpha} b_{k l}(\log \tau)^{l},
\end{aligned}
$$

are substituted into (3.6) and (3.7) yielding the following recursion relations for $f_{\alpha}(z)$ and $g_{v x}(z)$

$$
\begin{aligned}
f_{\alpha}^{\prime}(z)+(\alpha-1) f_{\alpha}(z)-g_{\alpha}(z) & =0, \\
y_{\alpha}^{\prime}(z)+(\alpha-2) y_{\alpha}(z)-B_{1} \frac{F^{(\alpha-3)}\left(t_{0}\right)}{(\alpha-3) !}-B_{0}+k g_{\alpha-1}(z) & \\
+p \sum_{\beta=1}^{\alpha-1} \sum_{\gamma=0}^{\beta} f_{\gamma}(z) f_{\beta-\gamma}(z) f_{\alpha-\jmath}(z)+p \sum_{\gamma=1}^{\alpha-1} f_{\gamma}(z) f_{\alpha-\gamma}(z) f_{0}+3 p f_{0}(z)^{2} f_{\alpha}(z) & =0,
\end{aligned}
$$


after shifting the indices of summation and separating the terms involving $f_{\alpha}(z)$.

Alternatively, in matrix form we obtain

$$
\begin{aligned}
& \left(\begin{array}{l}
f_{\alpha}(z) \\
g_{\alpha}(z)
\end{array}\right)^{\prime}+\left(\begin{array}{cc}
(\alpha-1) & --1 \\
3 p f_{0}^{2} & (\alpha-2)
\end{array}\right)\left(\begin{array}{l}
f_{\alpha}(z) \\
g_{\alpha}(z)
\end{array}\right) \\
& =\left(\begin{array}{c}
\text { or } \\
B_{1} \frac{F\left(z_{0}\right)^{(\alpha-3)}}{(\alpha-3) !}+B_{0}-k g_{\alpha-1}(z)-p \sum_{\beta=1}^{\alpha-1} \sum_{\gamma=0}^{\beta} f_{\gamma}(z) f_{\beta-\gamma}(z) f_{\alpha-\beta}(z)+p \sum_{\gamma=1}^{\alpha-1} f_{\gamma}(z) f_{\alpha-\gamma}(z) f_{0}
\end{array}\right) .
\end{aligned}
$$

$$
\mathbf{v}_{\alpha}^{\prime}(z)+A_{\alpha} \mathbf{v}_{\alpha}(z)=\mathbf{V}_{\alpha}(z)
$$

In order for this system to have valid logarithmic psi series solutions it must have integer resonances, which requires that the determinant of $A_{\alpha}, \alpha^{2}-3 \alpha+2+3 p f_{0}{ }^{2}$, have integer roots. We choose the values $B_{0}=0$ and $p f_{0}{ }^{2}=-2$, which is satisfied for $p=\frac{1}{2}$ and $f_{0}= \pm 2 i$, giving resonances at -1 and at 4 .

The convergence proof parallels that for the Lorenz system, found in section 2.3.

In order to prove the existence of a lower bound on the radii of convergence of the series, we first establish a bound on the coefficients of the series with the following proposition.

Proposition 5 For all $M, 0<M<1$. there exists $K, \frac{1}{\sqrt[\pi]{5}}<K<\infty$, such that for all $z<0$ and for all $\alpha \geq 5$.

$$
\left\|\mathrm{v}_{\alpha}(z)\right\|<M \frac{(2 K-K z)^{\alpha / k_{0}}}{\sqrt{\alpha+1}}
$$

where $k_{\mathrm{s}}=4$.

Proof. The proposition is a consequence of the following Assertion 4 if $N \geq\left(\frac{14 c}{1-28 \pi M^{2}}\right)^{2}+9$ where

$$
\begin{aligned}
c= & B_{1} A+k+2 \sum_{\gamma=1}^{4}\left|f_{\gamma}\right| \\
& +\sum_{\beta=5}^{8} \sum_{\gamma=\beta-4}^{4}\left|f_{\gamma}\right|\left|f_{\beta-\gamma}\right|+\sum_{\beta=\alpha-4}^{\alpha-1} \sum_{\gamma=\beta-\beta}^{\beta}\left|f_{\alpha-\beta}\right|\left|f_{\beta-\gamma}\right| \\
& +M\left(\pi+2 \pi \sum_{\gamma=0}^{4}\left|f_{\gamma}\right|+\frac{3}{\sqrt{6}} \sum_{\gamma=0}^{3}(4-\gamma)\left|f_{\gamma}\right|\right)-2 \sqrt{6} \pi M^{2},
\end{aligned}
$$


and if

$$
\left\|v_{\alpha}(z)\right\|<M \frac{(2 K-K z)^{\alpha / k_{a}}}{\sqrt{\alpha+1}}
$$

for all $\alpha<N$, then

$$
\left\|\mathrm{v}_{\alpha}(z)\right\|<M \frac{(2 K-K z)^{\alpha / k_{0}}}{\sqrt{\alpha+1}}
$$

for $\alpha=N$.

The proposition will then follow by induction.

The proof of the assertion follows that for the Lorenz psi series, with the following differences.

$P$ : the matrix of eigenvectors of $A_{\alpha}$, is

$$
\begin{gathered}
P=\left(\begin{array}{cc}
1 & 1 \\
2 & -3
\end{array}\right), \\
D_{\alpha}=P^{-1} A_{\alpha} P=\left(\begin{array}{cc}
\alpha+1 & 0 \\
0 & \alpha-4
\end{array}\right), \\
P(\sigma)=\|P\|\left\|P^{-1}\right\|=4
\end{gathered}
$$

and

$$
\begin{aligned}
G_{\alpha}= & B_{1} A+k+2 \sum_{\gamma=1}^{4}\left|f_{\gamma}\right| \\
& +\sum_{\beta=5}^{s} \sum_{\gamma=\beta^{-4}}^{4}\left|f_{\gamma}\right|\left|f_{\beta-\gamma}\right|+\sum_{\beta=\alpha-4}^{\alpha-1} \sum_{\gamma=\beta-4}^{\beta}\left|f_{\alpha-\beta}\right|\left|f_{\beta-\gamma}\right| \\
& +M\left(\pi+2 \pi \sum_{\gamma=0}^{4}\left|f_{\gamma}\right|+\frac{3}{\sqrt{6}} \sum_{\gamma=0}^{3}(4-\gamma)\left|f_{\gamma}\right|\right) \\
& +M^{2}(2 \pi \sqrt{a-9})-2 \sqrt{6} \pi M^{2}
\end{aligned}
$$

if we restrict the value of $K$ and $M$ such that

$$
r \geq \frac{1}{\sqrt[n]{5}},
$$

which can always be satisfied by sufficiently large $K$. 
The factor $\sqrt{\alpha-9}$ appears due to the term arising from the double sum

$$
p \sum_{\beta=1}^{\alpha-1} \sum_{\gamma=0}^{j} f_{\gamma}(z) f_{\beta-\gamma}(z) f_{\alpha-\beta}(z)
$$

which, after substitution of the bound gives an expression including

$$
\begin{aligned}
\sum_{\beta=10}^{\alpha-5} \sum_{\gamma=5}^{\beta-5} \frac{M^{3} K^{\alpha}}{\sqrt{\gamma+1} \sqrt{\beta-\gamma+1} \sqrt{\alpha-\beta+1}} & =M^{3} K^{\alpha} \sum_{\beta=10}^{\alpha-5} \frac{1}{\sqrt{\alpha-\beta+1}} \sum_{\gamma=5}^{\beta-5} \frac{1}{\sqrt{\gamma+1} \sqrt{\beta-\gamma+1}} \\
& \leq M^{3} K^{\alpha} \sum_{\beta=10}^{\alpha-5} \frac{1}{\sqrt{\alpha-\beta+1}} \sum_{\gamma=0}^{\beta} \frac{1}{\sqrt{\gamma+1} \sqrt{\beta-\gamma}} \\
& \leq \pi M^{3} K^{\alpha} \sum_{\beta=10}^{\alpha-5} \frac{1}{\sqrt{\alpha-\beta+1}} \\
& =\pi M^{3} K^{\alpha} \sum_{\beta=6}^{\alpha-9} \frac{1}{\sqrt{\beta}} \\
& \leq \pi M^{3} K^{\alpha} \int_{6}^{\alpha-9} \frac{1}{\sqrt{\beta}} d \beta \\
& =2 \pi M^{3} K^{\alpha}(\sqrt{\alpha-9}-\sqrt{6}) .
\end{aligned}
$$

Combining all the terms not involving $\alpha$ in the constant $c$ we have

$$
G_{\alpha} \leq M^{i} 2 \pi \sqrt{\alpha-9}+c
$$

Recall that we are attempting to show that

$$
\left\|\mathrm{v}_{\alpha}(z)\right\|<M \frac{(2 K-K z)^{\alpha / k_{0}}}{\sqrt{\alpha+1}},
$$

for $\alpha=N$. This requires that the inequality

$$
\frac{\tilde{J} P(\sigma) G_{\alpha x}}{2(\alpha-4)} \leq \frac{1}{\sqrt{\alpha+1}}
$$

be satisfied for $\alpha=N$.

Substituting $G_{\alpha}$ into (3.13) we obtann

$$
\frac{14\left(2 \pi H^{2} \sqrt{\alpha-1}+c\right)}{(\alpha-4)} \leq \frac{1}{\sqrt{\alpha+1}} .
$$


Since $\frac{w \alpha-4}{v a-1} \geq \sqrt{\alpha-9}$, in order for this condition to hold for $\alpha=N$, we must have

$$
N \geq\left(\frac{14 c}{1-28 \pi M^{2}}\right)^{2}+9 .
$$

The assertion and thus the proposition follow.

The comparison test can be applied as for the Lorenz psi series to obtain an estimate of the lower bound on the radii of convergence of the series. 
Chapter 4

\section{Figures}




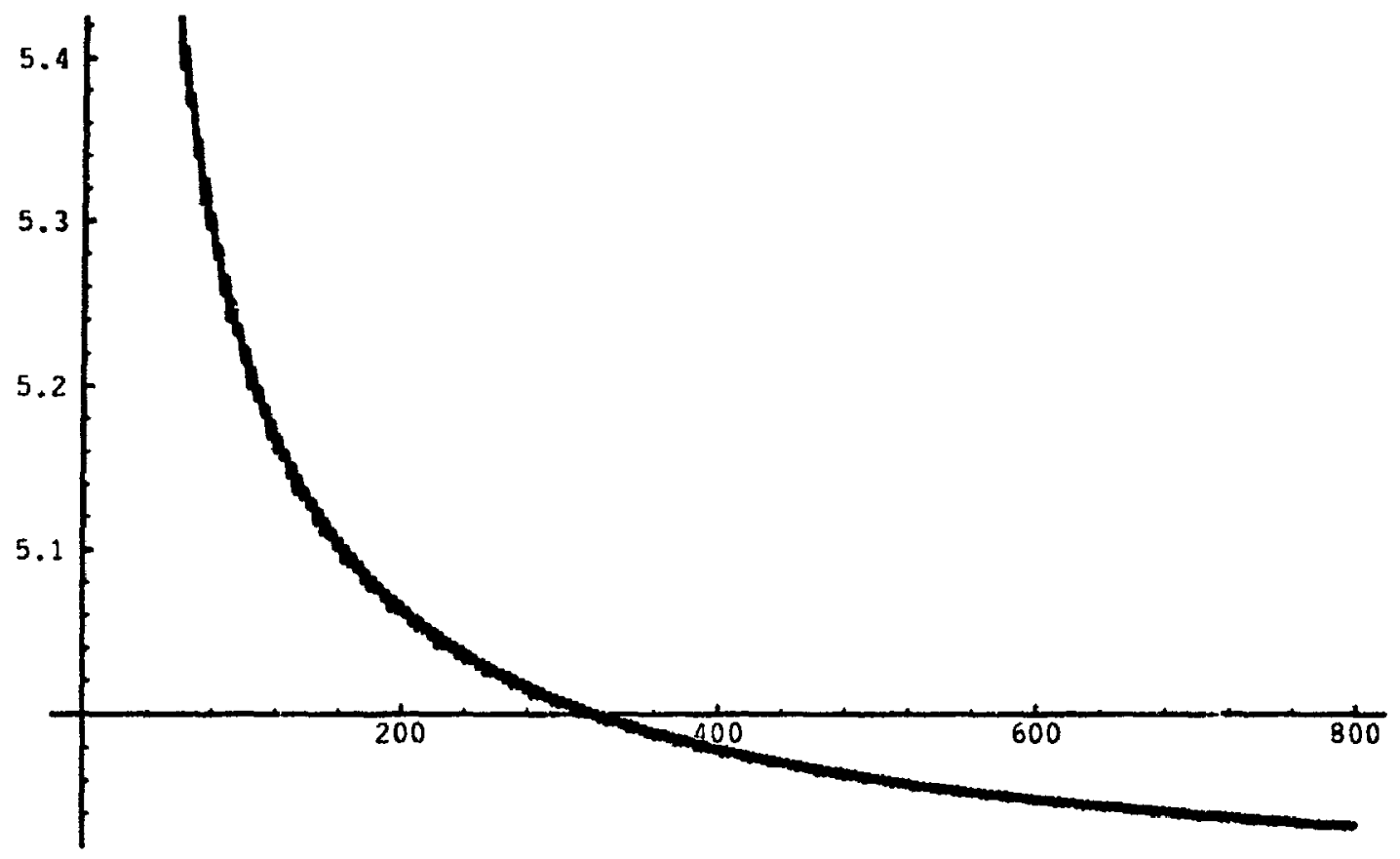

Figure 4.1: $L(\alpha)$ versus $\alpha$ for the ordinary series solution of the Lorenz system 


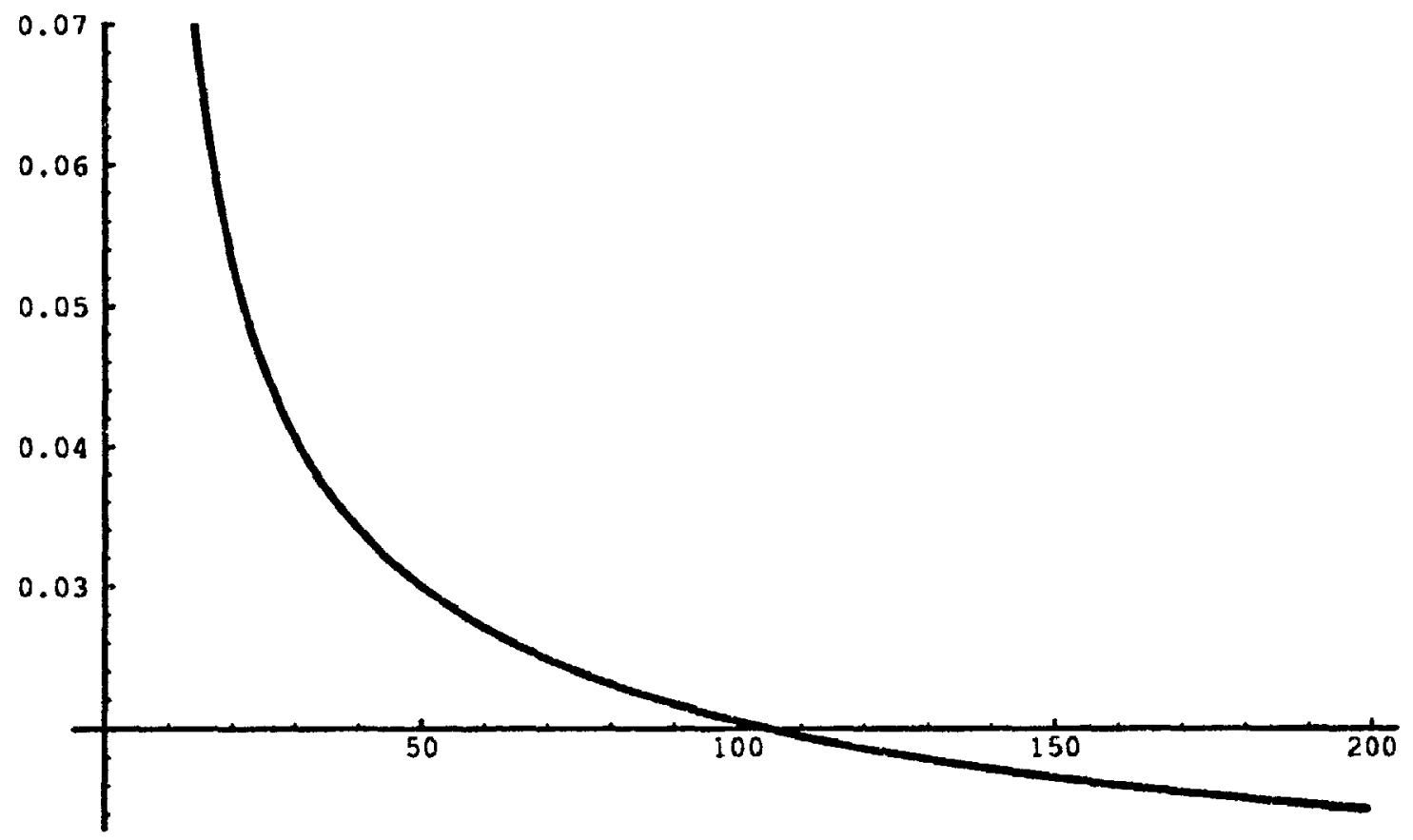

Figure 4.2: $\mathcal{R}_{\text {est }}$ versus $R$ for the ordinary series solutions of the Lorenz system 


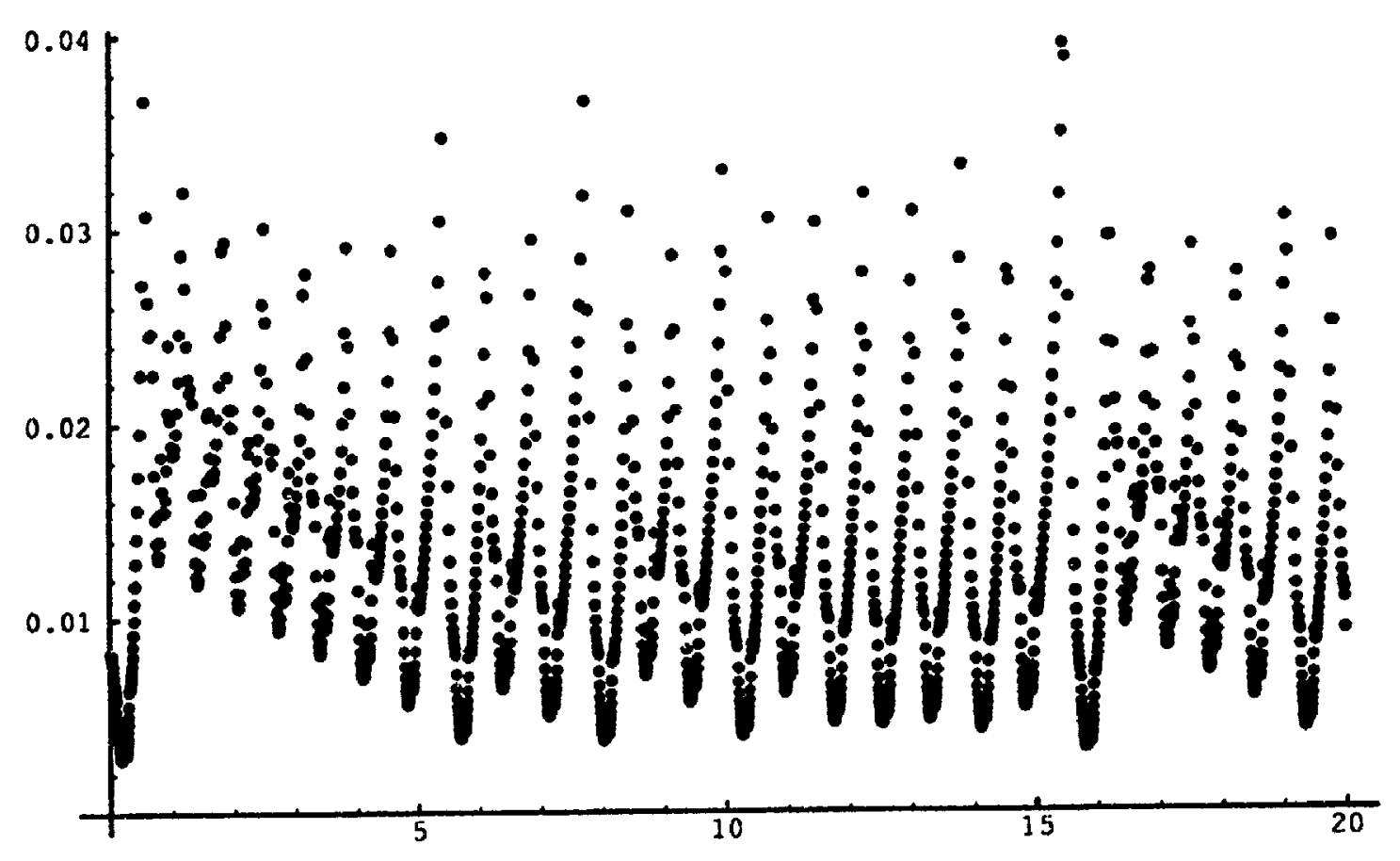

Figure 4.3: $\mathcal{R}_{\text {est }}$ versus time for the ordinary series solution for $x(t)$ of the Lorenz system 


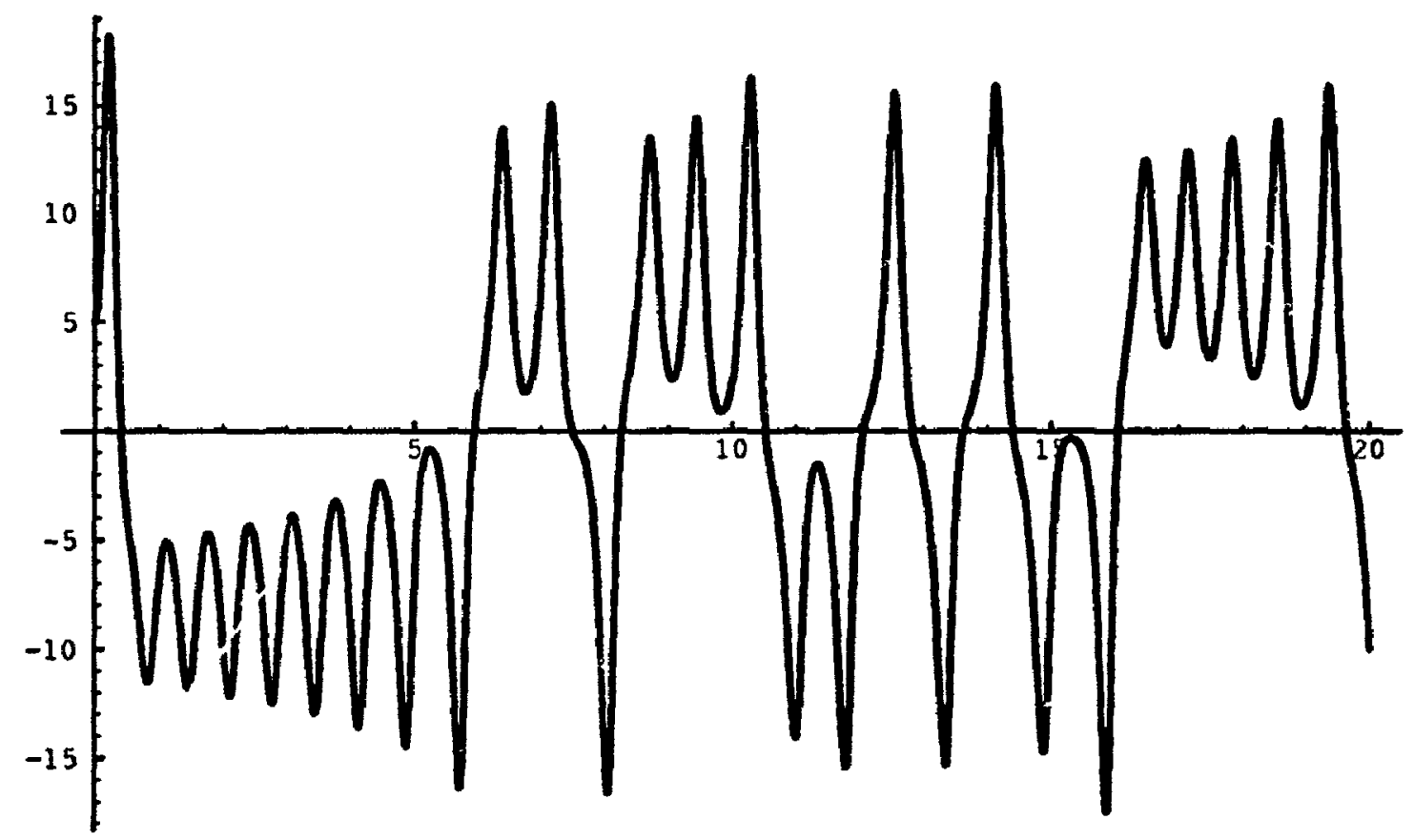

Figure 4.4: Ordinary series solution for $x(t)$ of the Lorenz system, truncated at 20 terms, versus time 


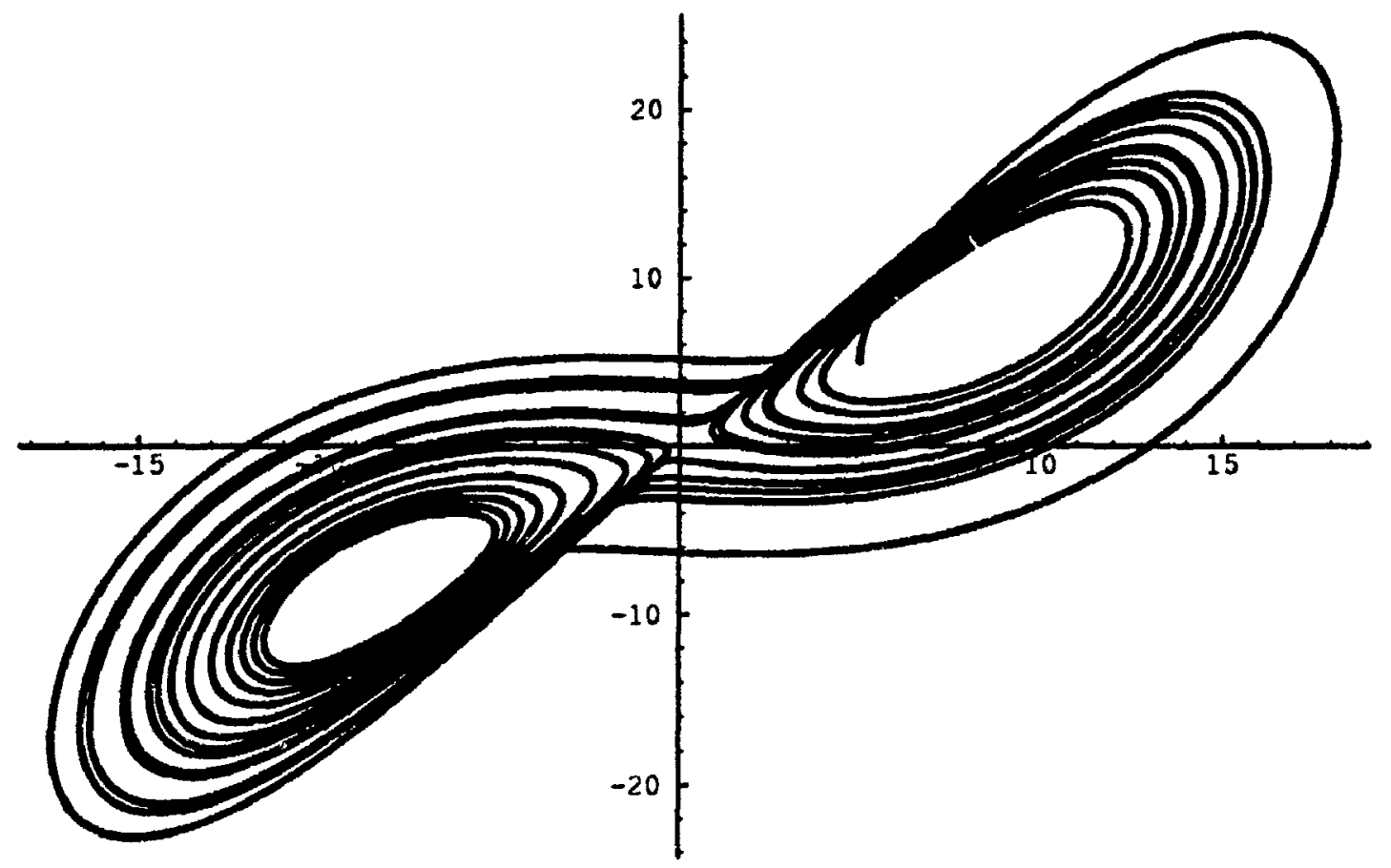

Figure 4.5: Projection of the attractor on the $z$ plane produced by the ordinary series solutions of the Lorenz system 


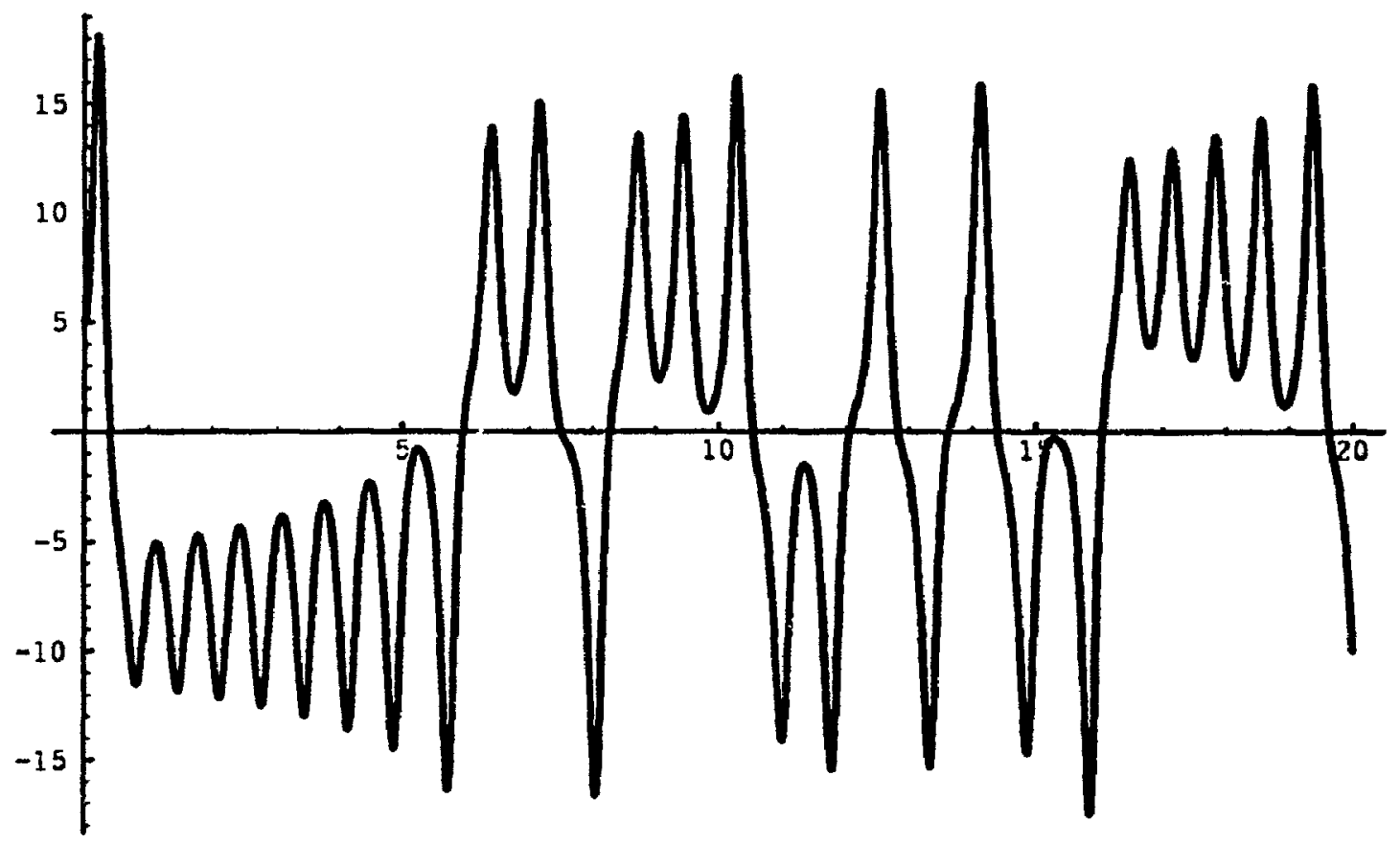

Figure 4.6: $x(t)$ of the Lorenz system versus time, obtained by numerical integration 


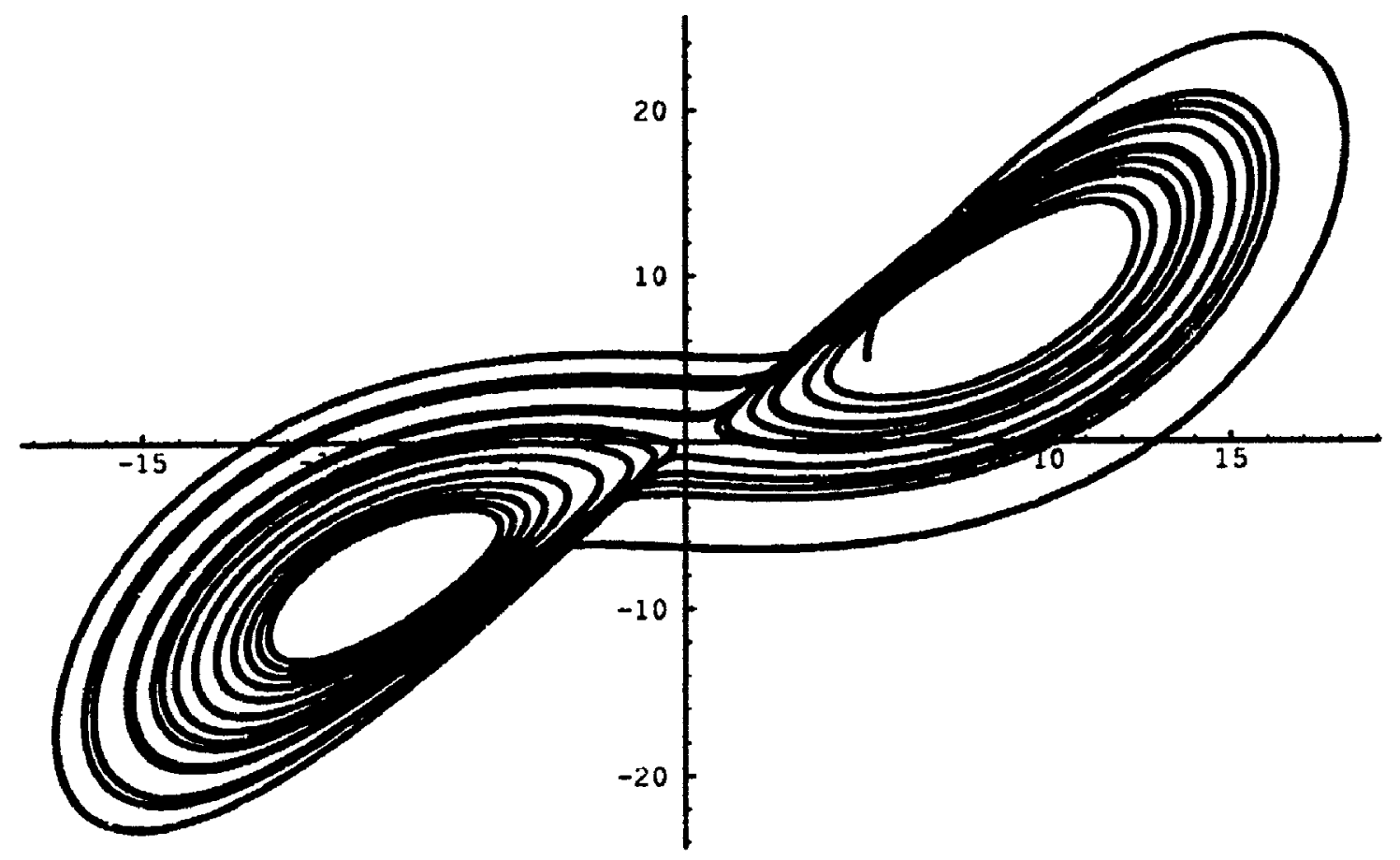

Figure 4.7: Projection of the attractor on the $z$ plane for the Lorenz system, obtained by numerical integration 


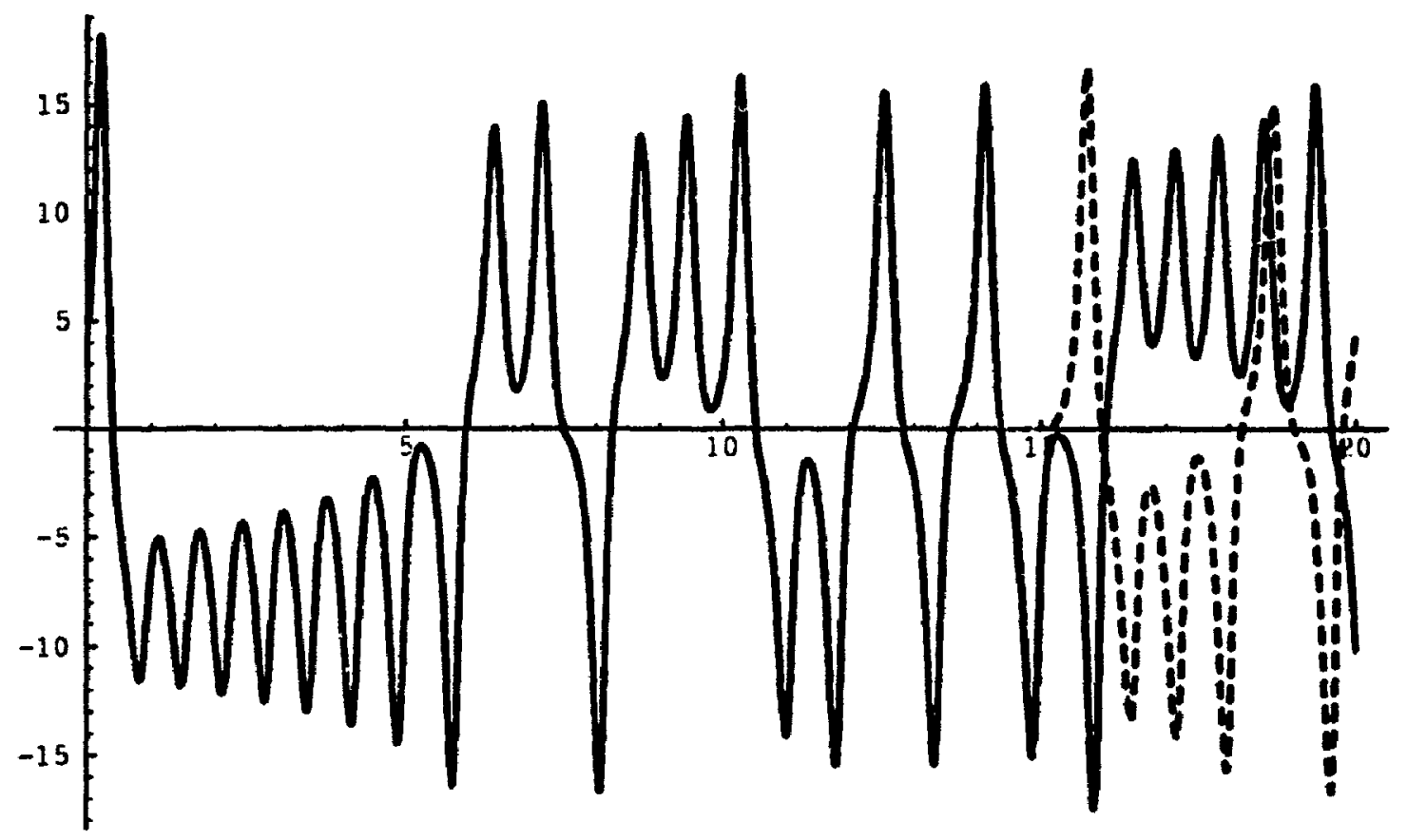

Figure 4.8: $x(t)$ versus time for the ordinary series of the Lorenz system, initial conditions $(5,5,5)$ (solid) and $(5.0001,5,5)$ (dashed) 


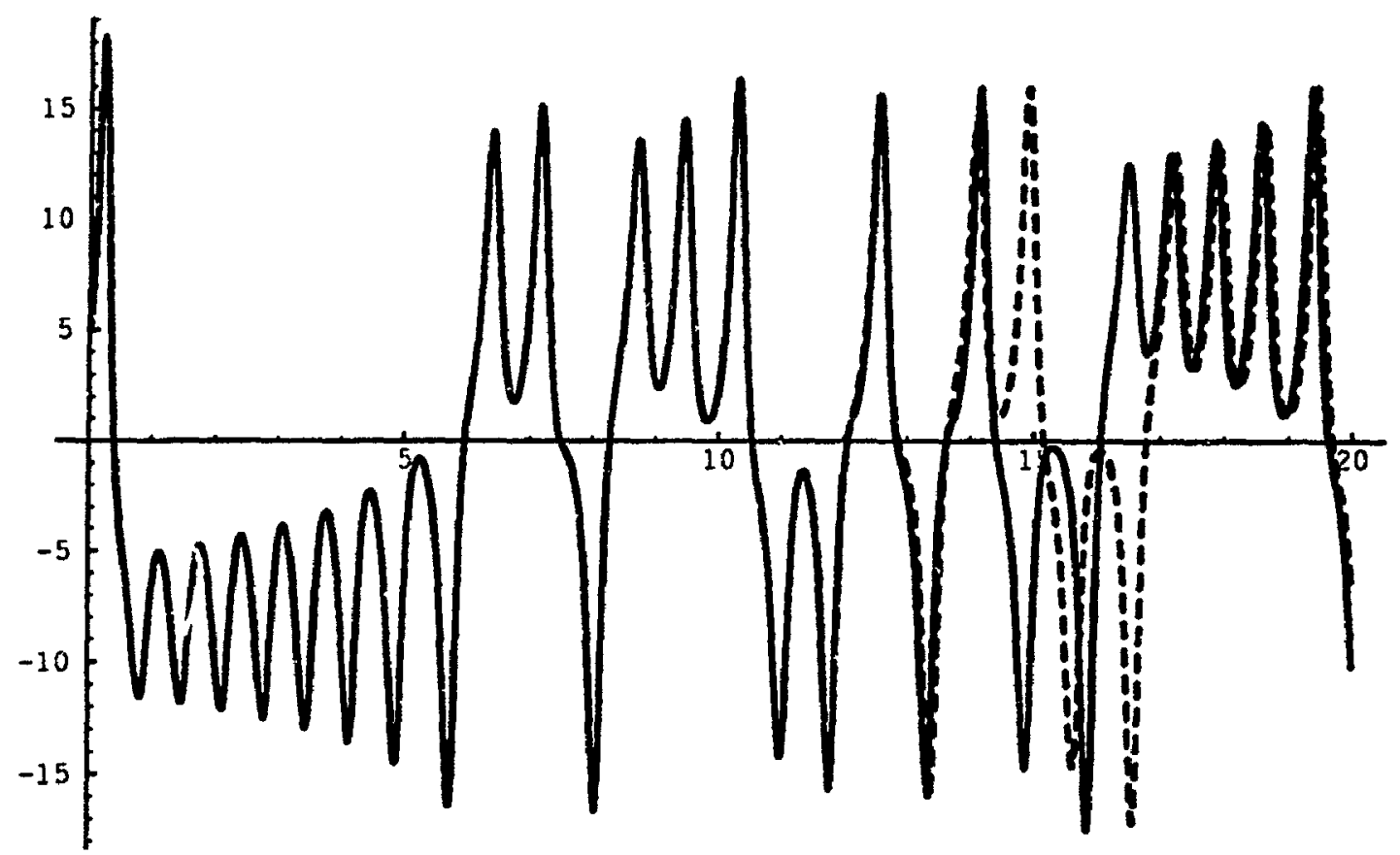

Figure 4.9: $x(t)$ versus time for the ordinary series of the Lorenz system, initial conditions $(5.5,5)$ (solid) and $(5.001,5,5)$ (dashed) 


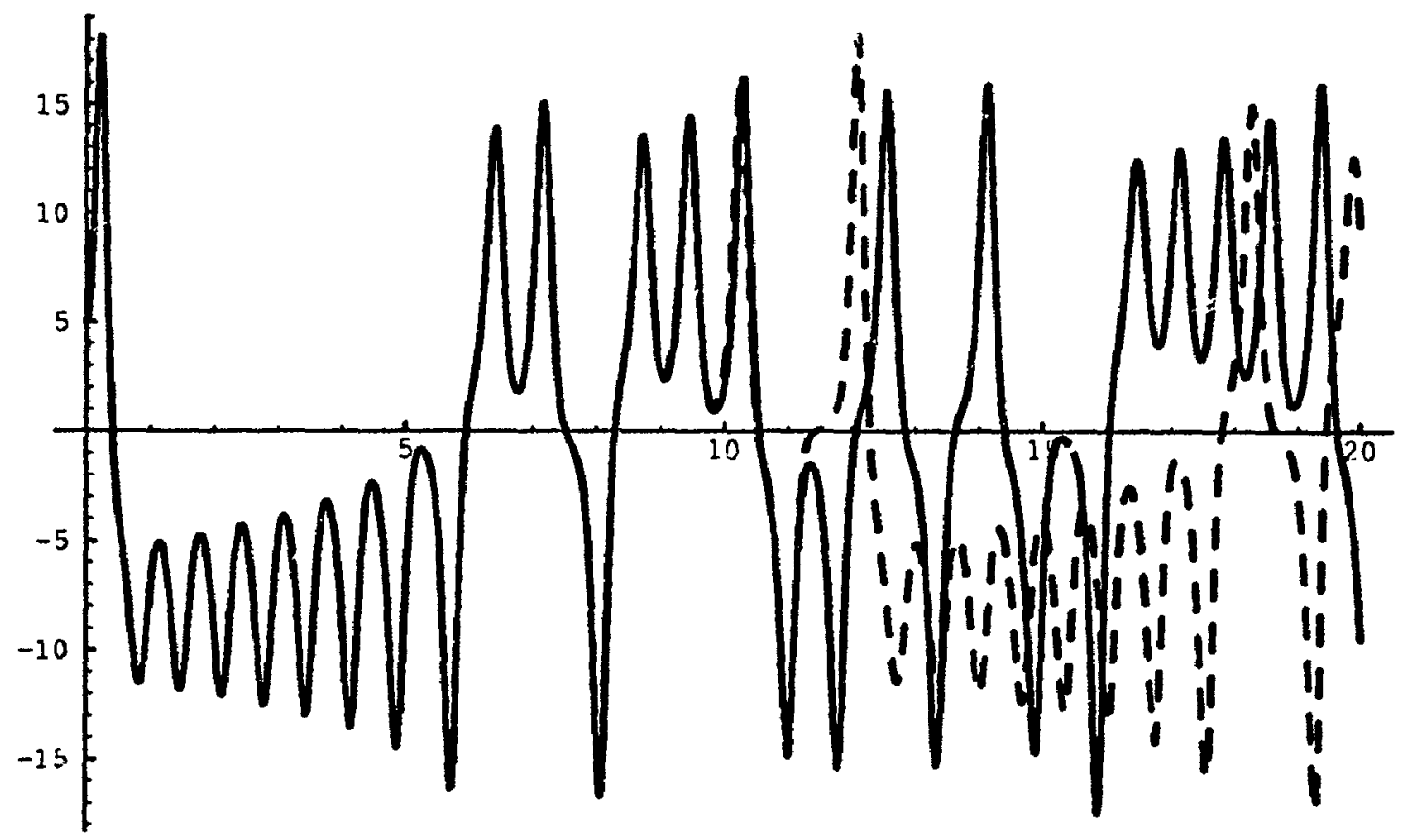

Figure 4.10: $x(t)$ versus time for the ordinary series of the Lorenz system, initial conditions $(5,5,5)$ (solid) and $(5.01,5,5)$ (dashed) 


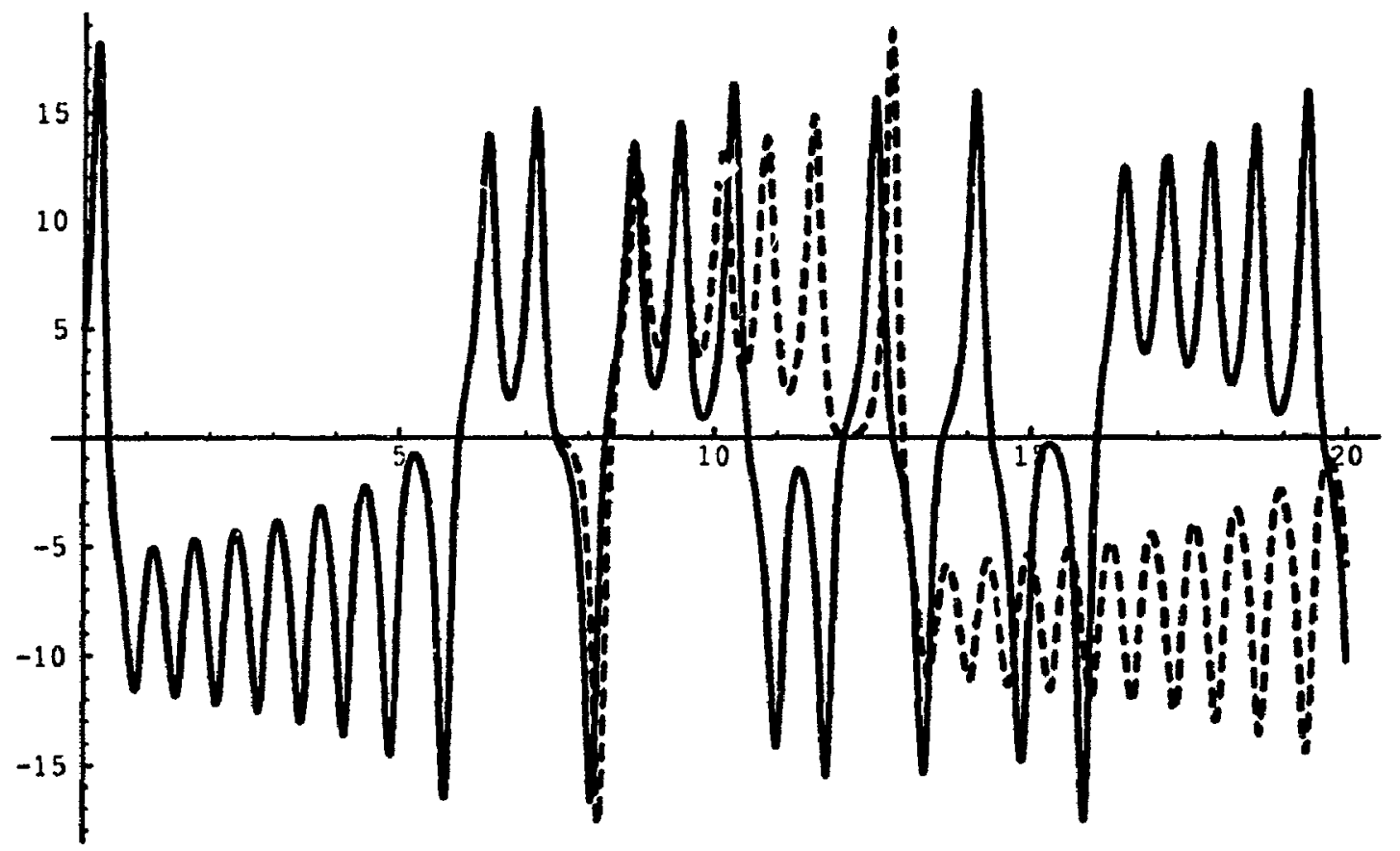

Figure 4.11: $x(t)$ versus time for the ordinary series of the Lorenz system, initial conditions $(5,5,5)$ (solid) and $(5.1,5,5)$ (dashed) 


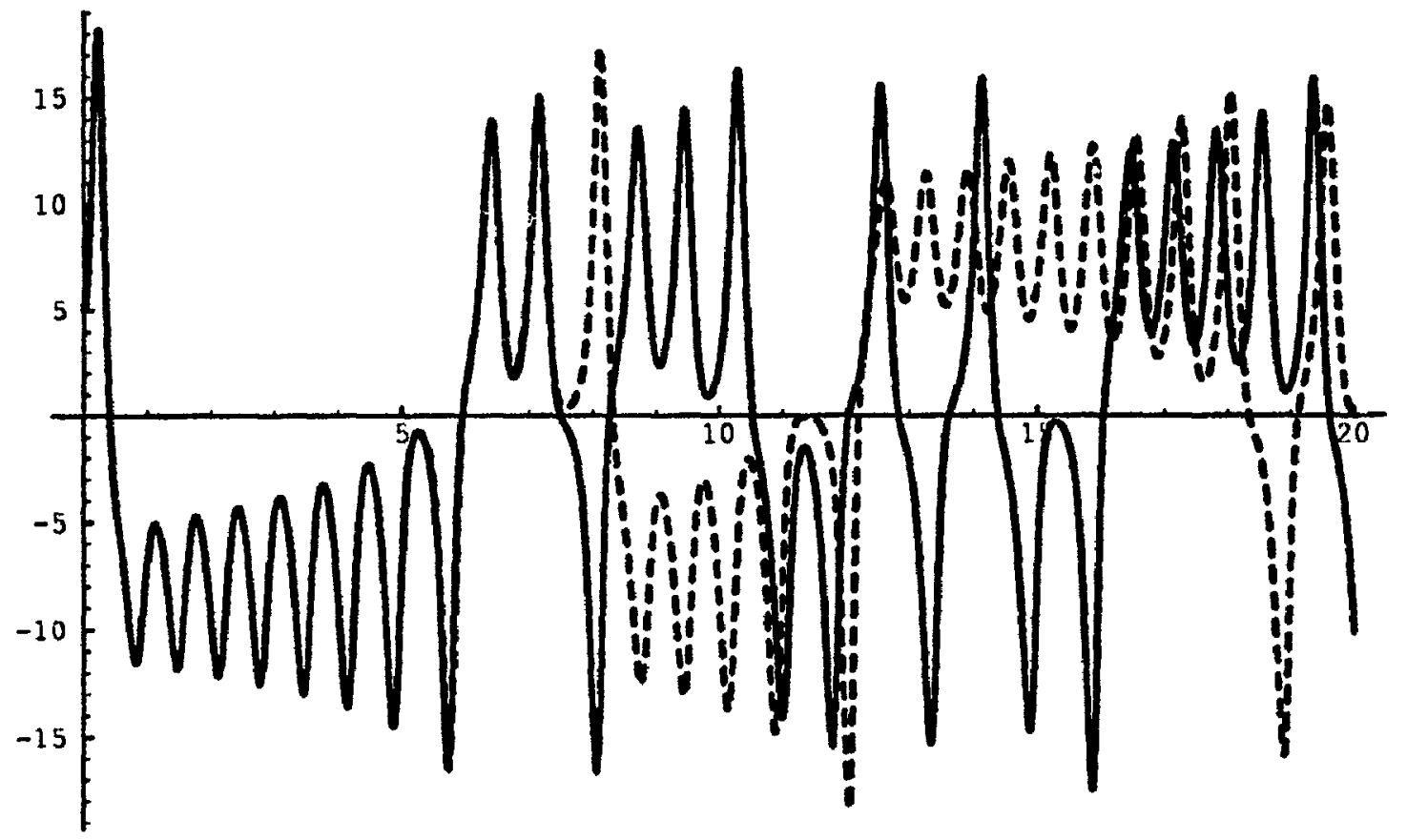

Figure 4.12: $x(t)$ versus time for the ordinary series of the Lorenz system, initial conditions $(5,5,5)$ (solid) and $(5.2,5,5)$ (dashed) 


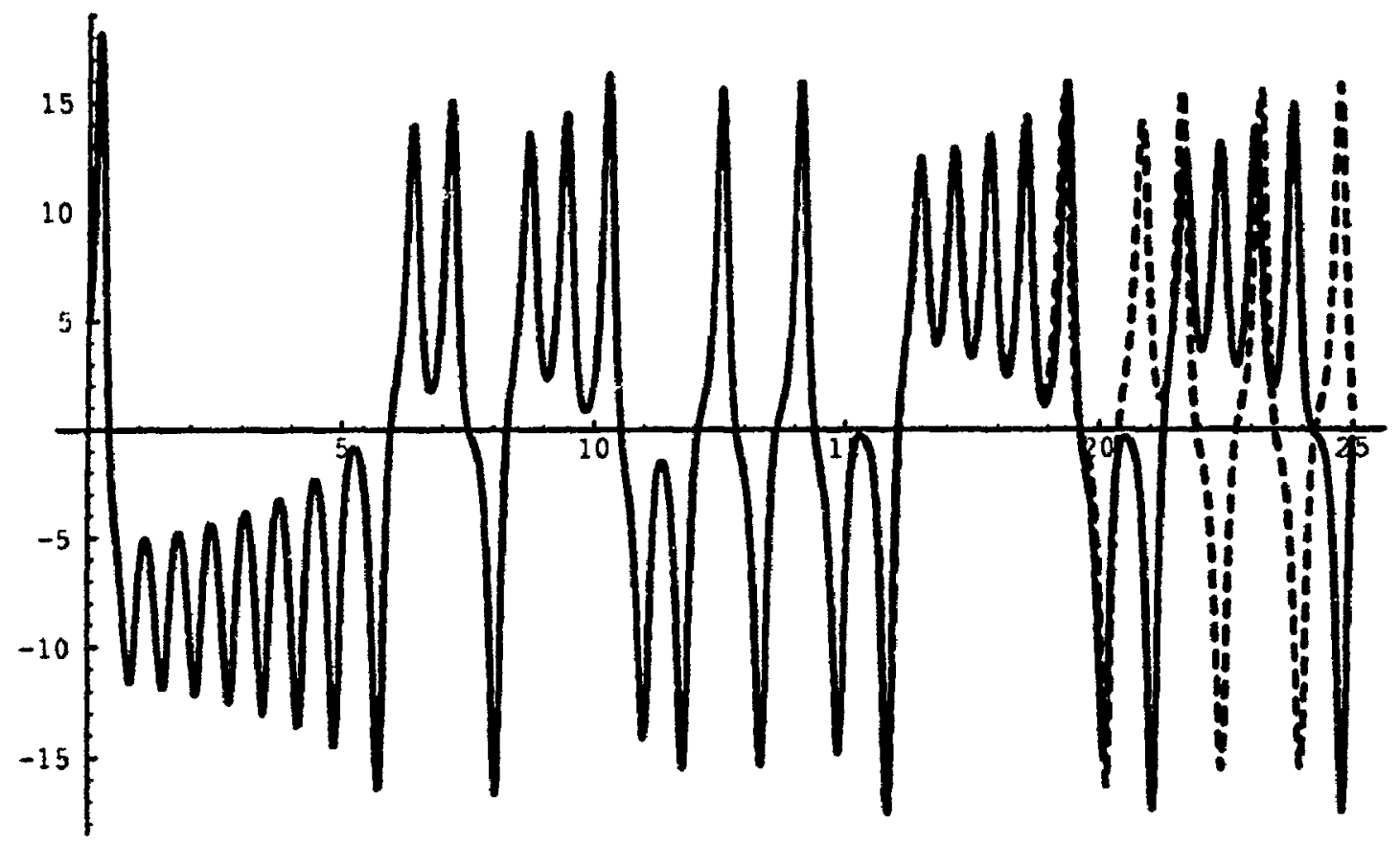

Figure 4.13: $x(t)$ versus time for the ordinary series of the Lorenz system, initial conditions $(-13.9,-15.8568,32.1233)$ at $t=10.9785$ (dashed) and $(5,5,5)$ at $t=0$ 


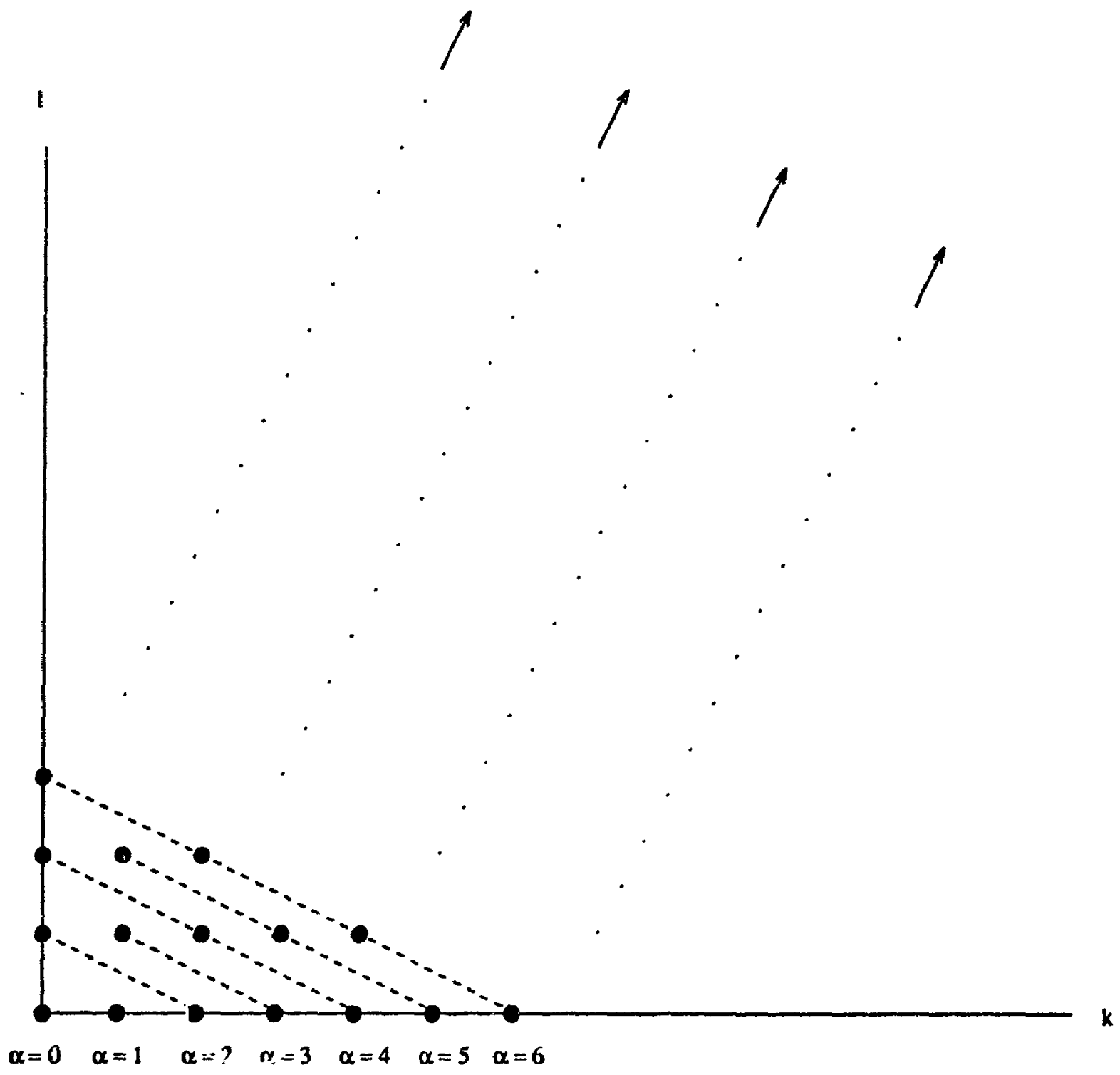

Figure 4.14: Resummation of the double series along the lines $\alpha=k+2 l$ 


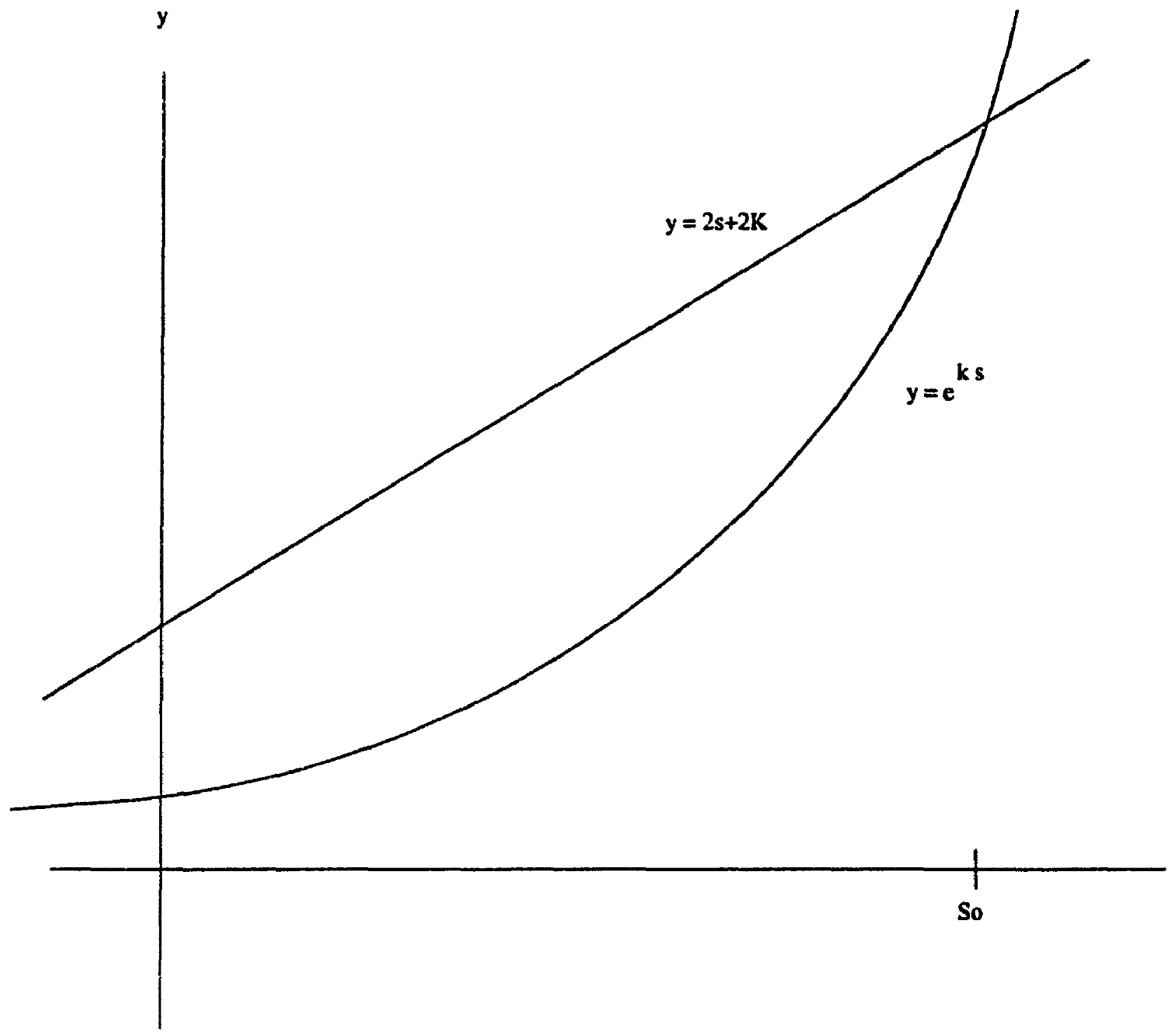

Figure 4.15: Solution of the inequality $e^{k_{0} s}>2 K+K s$ 
CIAPTER 4. FIGURES

kh us. dipha

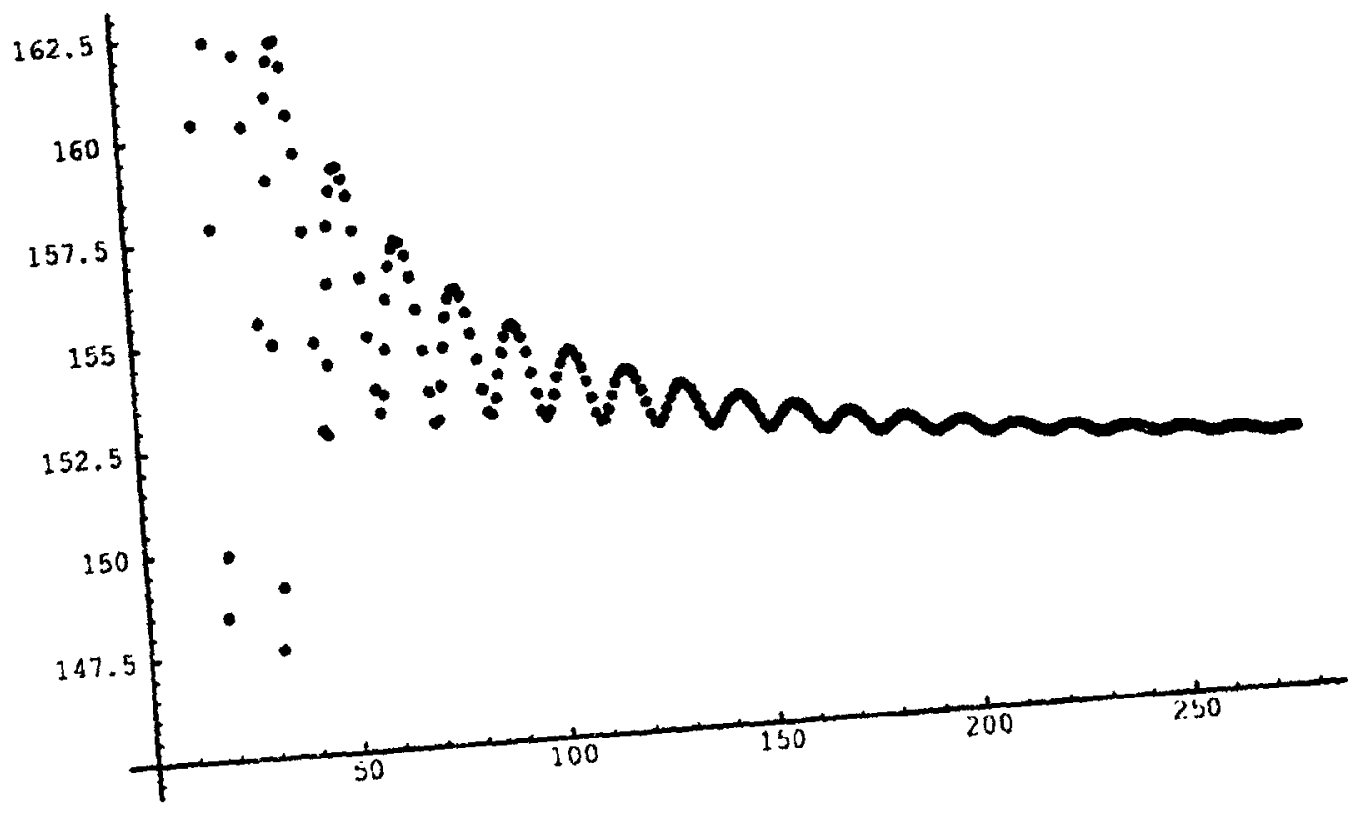

Figure 4.16: $L(\alpha)$ vs $a$ for the psi series solution of the lorenz system 


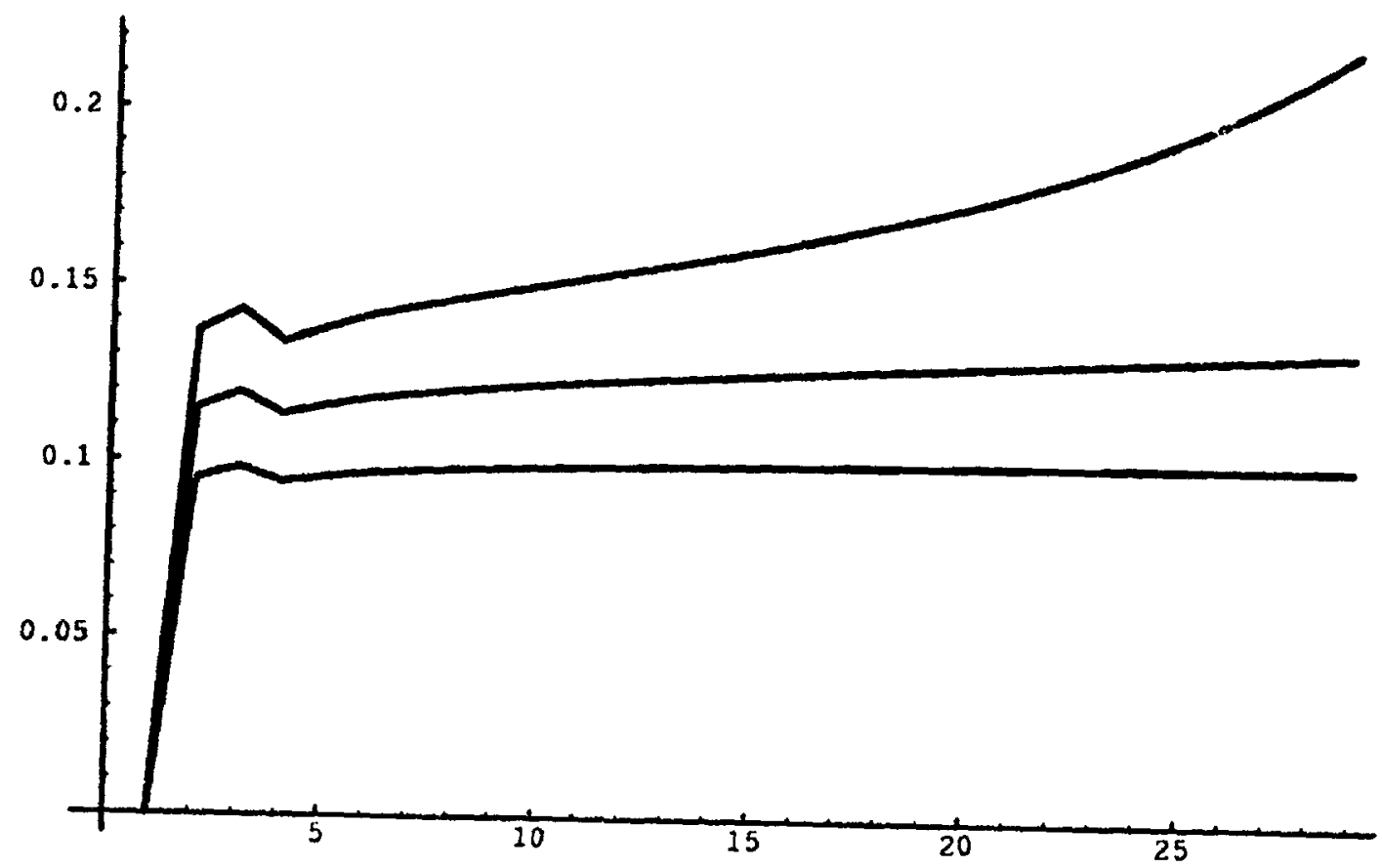

Figure 4.17: The psi series for $x(t)$ of the Lorenz systern versus the truncation point, $t=0.9, t=1.0$ and $t=1.1$ (top to bottom) 


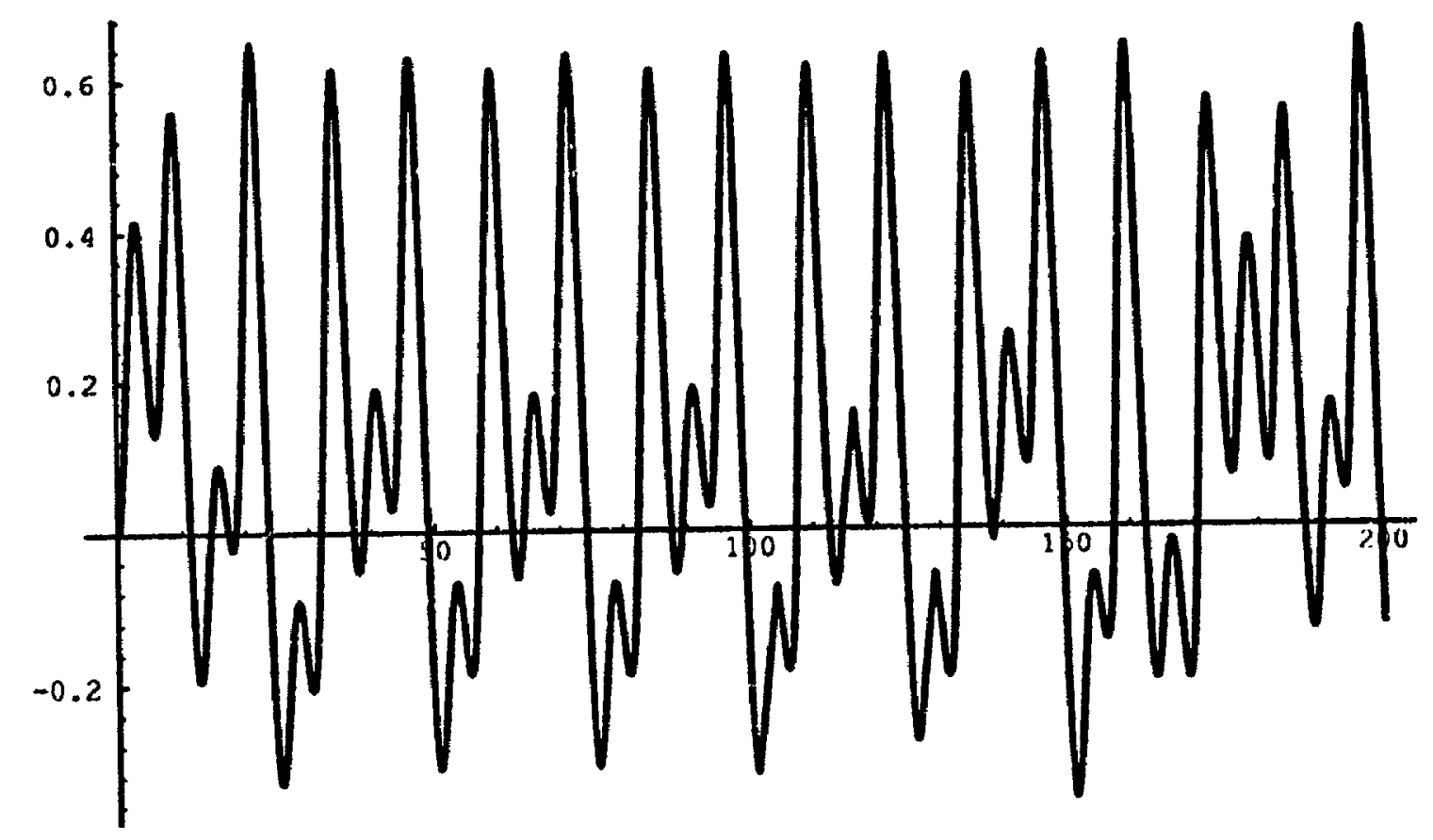

Figure 4.18: The ordinary series for $r(t)$ of the Duffing equation, truncated at 25, terms, versus time 


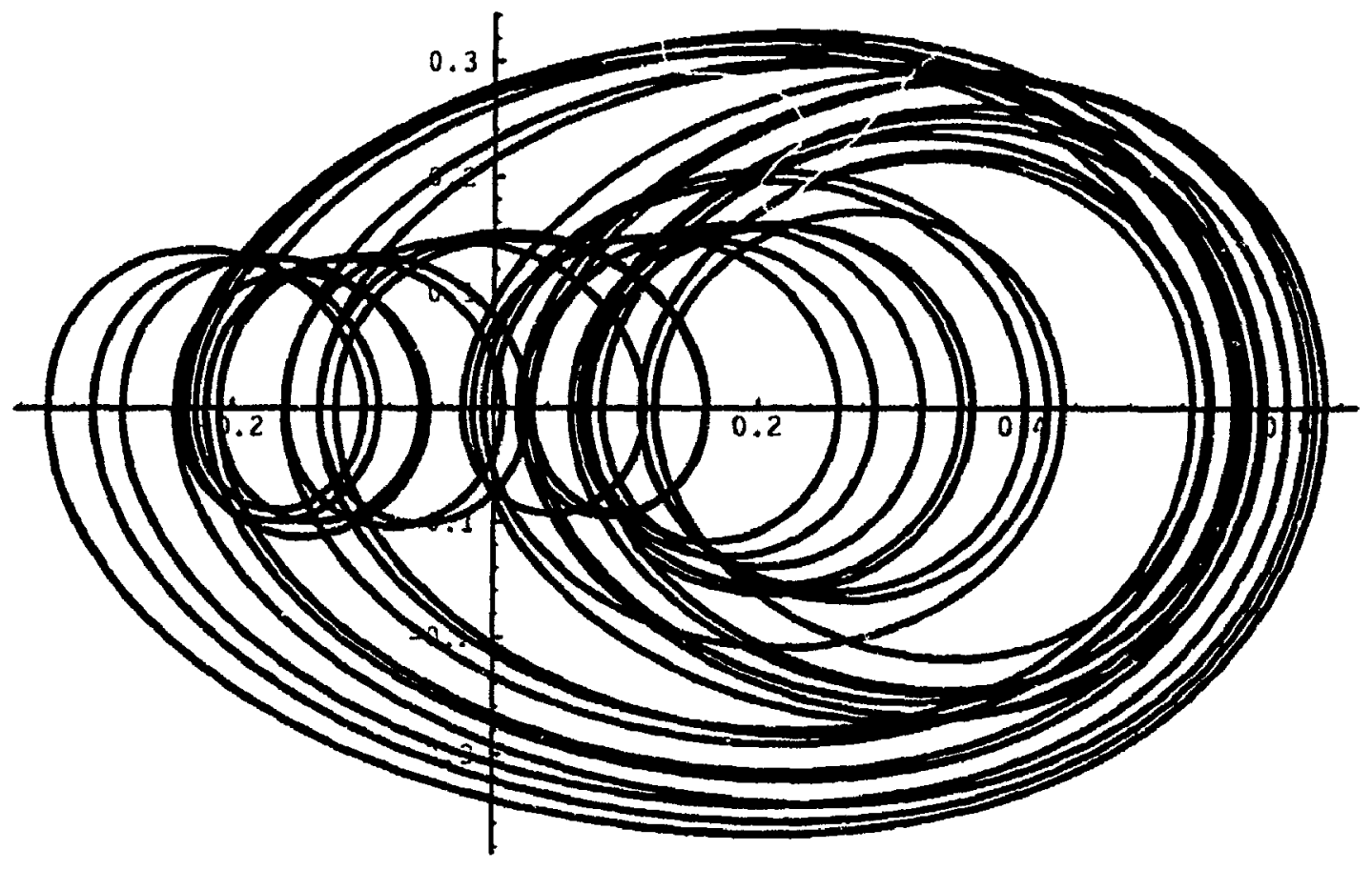

Figure 4.19: The ordinary series for $x(t)$ versus $x^{\prime}(t)$ of the Duffing equation, truncated at 25 terms 


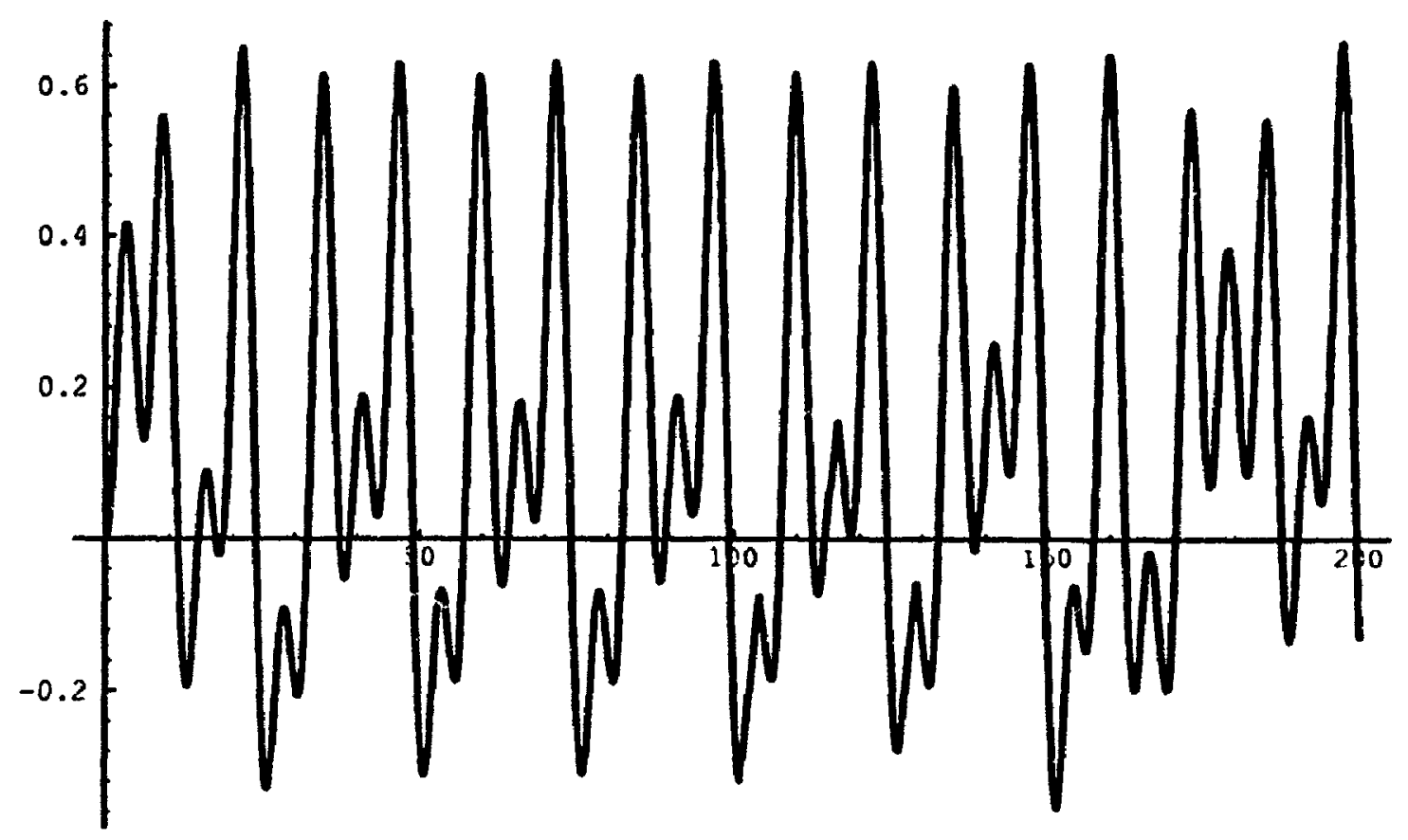

Figure 4.20: $x(t)$ of the Duffing equation versus time, obtained by numerical integration 


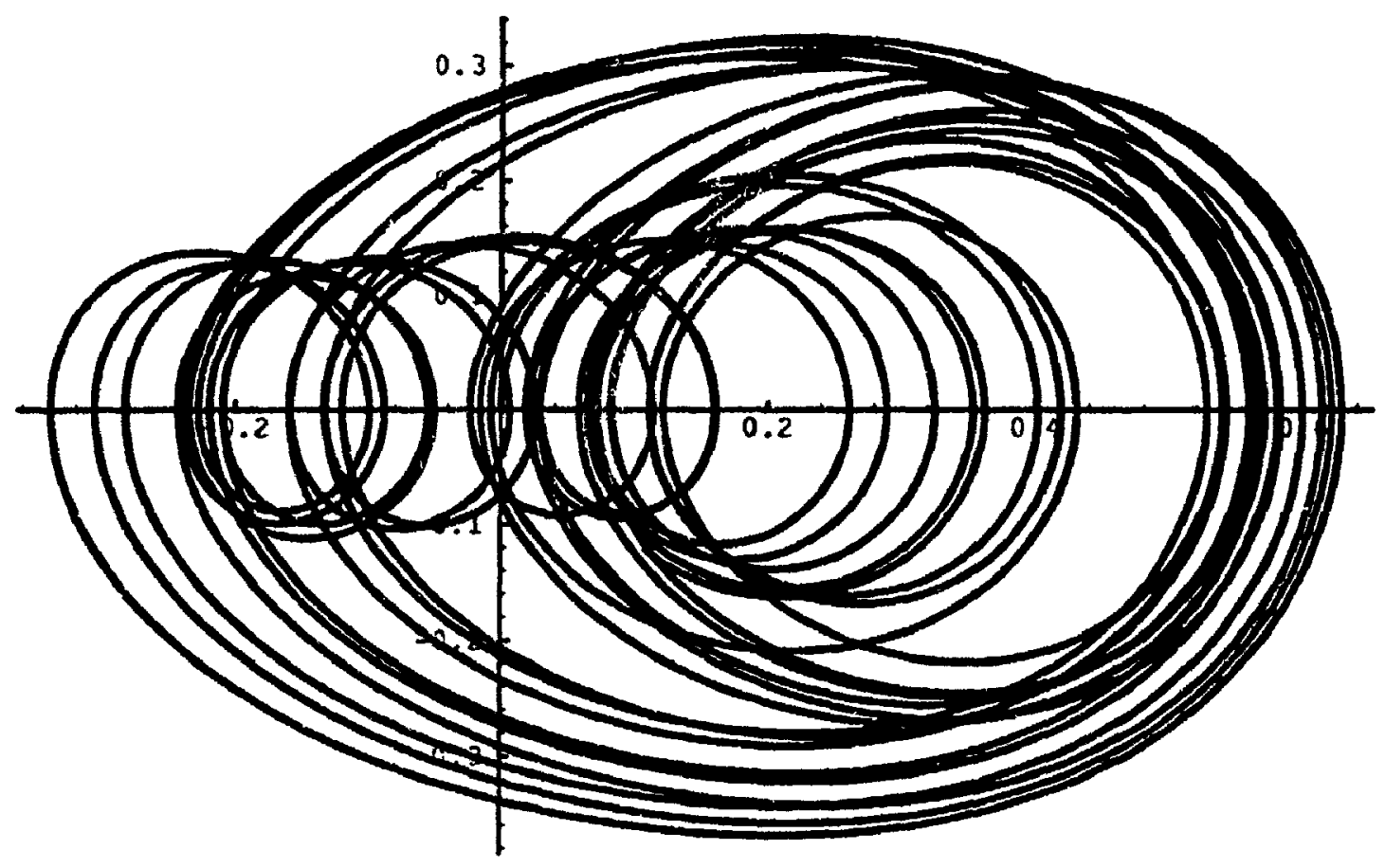

Figure 4.21: $x(t)$ versus $x^{\prime}(t)$ of the Duffing equation, obtained by numerical integration 


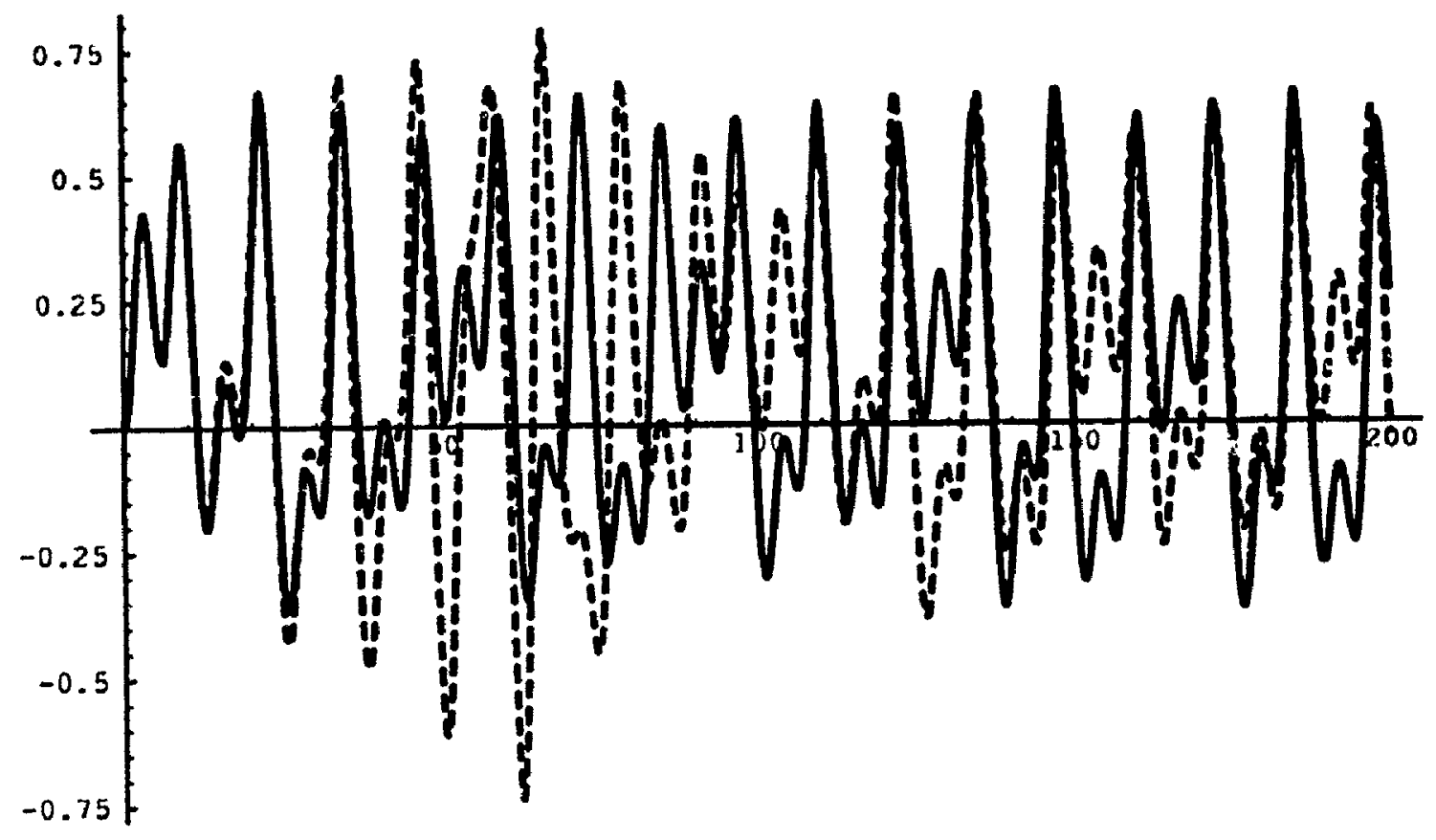

Figure 4.22: $x(t)$ versus $t$ for the ordinary series of the Duffing equation, initial conditions $(0,0)$ (solid) and $(0.01,0,0)$ (dashed) 


\section{Chapter 5}

\section{Summary and Conclusions}

In this thesis we have formed series solutions about non-singular points, poles and logarithmic branch points, for the Lorenz system and the Duffing equation. For each series it was proved that a lower bound on the radius of convergence exists. Previously the convergence of the psi series for these systems was unknown. The bound was calculated for various values of the system's parameters, and found to be generally within an order of magnitude of the actual radii of convergence of the series.

For both systems, the ordinary series were continued by re-expanding the series within their radii of convergence. These solutions were shown to provide plots of the trajectories identical to those ohtained by numerical integration.

Using the ordinary series solutions for the Lorenz system, it was observed that as the difference in initial conditions between two neighbouring trajectories increased, their point of divergence decreased.

For the psi series solutions for the Lorenz system it was calculated that the lower bound on the radii. when the logarithmic terms entered at the fourth term, was higher than when the logarithmic terms entered at the second term. The actual radius was observed to follow the same pattern. This is interesting in view of the observations made by cuher researchers. for the governing equations of anharmonic oscillators [10], that that the higheri the order at which the logarithmic singularities enter, the smaller the chaotic region.

For the psi series solutions for the Duffing equation it was calculated that the 
lower bound on the radii decreased as the strength of the forcing increased. This supports observations made by other researct $s[5,7,52,55]$ that as the motion became more chaotic, the singularity pattern condensed and the structures gradually collapsed towards the real axis $[5,7,52,55]$.

At the conclusion of the thesis several questions naturally suggest themselves.

Firstly, after applying this method of proof to these two systems several observations can be made. For ordinary differential equations of the form

$$
u^{(n)}=L\left(t, u^{1}, \cdots\right)+P(u),
$$

where $P$ is a polynomial function and $L$ is a linear function, one can see that if $P$ is at most of third order, this method of proof will be successful.

When $P$ is permitted to be of the fourth order, it is not obvious how to modify the proof so that it will succeed. The difficulty sccurs in the condition on $N$. The term similar to (3.12) will be a triple sum rather than a double sum. $G_{\alpha}$ will be of the from $M^{3}(\alpha-C)^{\frac{3}{2}}+$ constants, where $C$ is some positive integer, due to the double integration of $\frac{1}{\sqrt{\alpha}}$.

The inequality that $N$ must satisfy for the induction process to succeed will appear as

$$
M^{3}\left(\alpha-()^{\frac{3}{2}} \leq(\alpha-C)^{\frac{1}{2}}\right.
$$

or

$$
x \leq \frac{1}{M^{3}}+C,
$$

which makes the proof unsuccess'ul.

Secondly, an estimate of an upper bound on the radii of convergence would be desirable. However, for all the series, the coefficients appear to show no pattern in their signs, and thus how an upper bound could be estimated is unclear. Do the sequence of signs give us any information regarding the integrability of the system?

Lastly, T.C. Bountis et. al [3. 4] 'have found that those systems which satisfy the weak Painlevé property and are integrable have finitely sheeted solutions, whoreas those which are not :ntegrable have infinitely steeted solutions. Ilow do the radii of convergence of their psi series solutions compare? 
To conclude. series solutions of nonlinear differential equations in the complex plane may possibly lead to insight into why certain singularity structures lead to integrability, and others do not. 
Appendix A Derivation of the formulae for $f_{10}(z), g_{\alpha}(z)$ and $h_{\alpha}(z)$ for the Psi Series of the Lorenz equations

From (2.40) we have

$$
\mathrm{V}_{\alpha}(z)=\int_{-\infty}^{z} P e^{D_{u}(q-z)} P^{-1} \mathrm{~V}_{\alpha}(q) d q
$$

where

$$
\begin{gathered}
\mathbf{v}_{\alpha}(z)=\left(\begin{array}{c}
f_{\alpha}(z) \\
y_{\alpha}(z) \\
h_{\alpha}(z)
\end{array}\right), \\
\mathbf{V}_{\alpha}(z)=\left(\begin{array}{c}
-\sigma f_{\alpha-1}(z) \\
R f_{\alpha-2}(z)-g_{\alpha-1}(z)-\sum_{\beta=1}^{\alpha-1} f_{\alpha-\beta}(z) h_{\beta}(z) \\
\sum_{\beta j=1}^{\alpha-1} f_{\alpha-\beta}(z) g_{\beta}(z)-B h_{\alpha-1}(z)
\end{array}\right),
\end{gathered}
$$

$P$, the matrix of eigenvectors of $A_{\alpha}$, is given by

$$
P=\left(\begin{array}{ccc} 
\pm \sigma / 2 & \mp \sigma & \pm \sigma / 2 \\
\mp 1 & \mp 1 & \pm 3 / 2 \\
i & i & i
\end{array}\right)
$$

and

$$
D_{\alpha}=P^{-1} A_{\alpha x} P=\left(\begin{array}{ccc}
\alpha+1 & 0 & 0 \\
0 & \alpha-\vartheta & 0 \\
0 & 0 & \alpha-4
\end{array}\right) \text {. }
$$

After multiplying the four matrices under the integral sign together, we chtain

$$
V_{i x}(z)=\int_{-\infty}^{z} T\left(\begin{array}{c}
e^{(\alpha+1)(y-z)} \\
e^{(\alpha-2)(q-z)} \\
e^{(\alpha-4)(q-z)}
\end{array}\right) d q,
$$

where

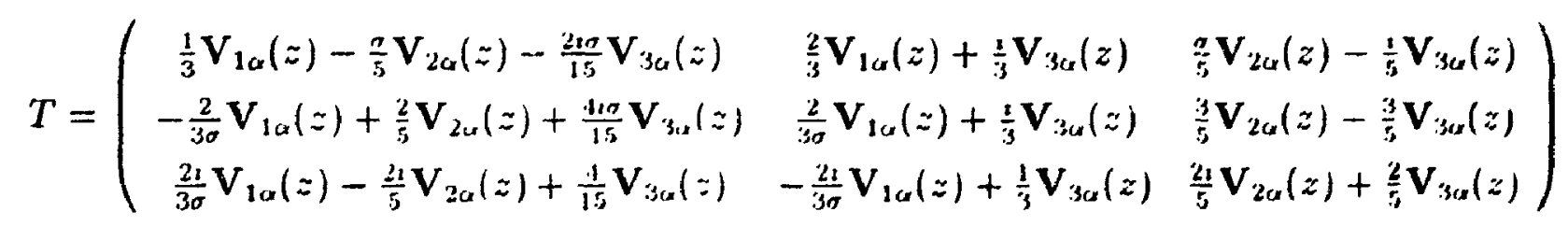


Letting $t$ denote an entry in $T$, we must now find an expression for

$$
\int_{-\infty}^{z} e^{\lambda(q-z)} t(q) d q
$$

where $\lambda$ equals $\alpha+1, \alpha-2$ or $\alpha-4$.

Note that each entry in $T$ is a function of $f_{\alpha}(z), g_{\alpha}(z)$ and $h_{\alpha}(z)$ and therefore is a polynomial in $z$ of order less than or equal to $\left\lfloor\frac{\alpha}{2}\right\rfloor$.

Integrating (5.3) by parts yields the following, if $e^{\lambda(q-z)}$ evaluated at $-\infty$ vanishes (which is true for $\alpha>4$ )

$$
\int_{-\infty}^{z} e^{\lambda(q-z)} t(q) d q=\frac{1}{\lambda} t(z)-\frac{1}{\lambda} \int_{-\infty}^{z} e^{\lambda(q-z)} t^{\prime}(q) d q .
$$

After $n$ integration by parts we obtain

$$
\int_{-\infty}^{z} e^{\lambda(q-z)} t(q) d q=\sum_{k=0}^{n-1} \frac{(-1)^{k} t^{(k)}}{\lambda^{(k+1)}}+\frac{(-1)^{n}}{\lambda^{n}} \int_{-\infty}^{z} e^{\lambda(q-z)} t^{(n)}(q) d q .
$$

If we let $n-1=\left\lfloor\frac{\alpha}{2}\right\rfloor$, then $t^{(n)}=0$, and

$$
\int_{-\infty}^{z} e^{\lambda(q-z)} t(q) d q=\sum_{k=0}^{\left\lfloor\frac{\alpha}{2}\right\rfloor} \frac{(-1)^{k} t^{(k)}}{\lambda^{(k+1)}} .
$$

Therefore, for $\alpha>4$, we obtain the following equations for $f_{\alpha}(z), g_{\alpha}(z)$ and $h_{\alpha}(z):$

$$
\begin{aligned}
& f_{\alpha}(z)=\sum_{k=0}^{\left\lfloor\frac{a}{2}\right\rfloor}(-1)^{k}\left(\frac{t_{11}^{(k)}(z)}{(\alpha+1)^{(k+1)}}+\frac{t_{12}^{(k)}(z)}{(\alpha-2)^{(k+1)}}+\frac{t_{13}^{(k)}(z)}{(\alpha-4)^{(k+1)}}\right), \\
& g_{\alpha}(z)=\sum_{k=0}^{\left\lfloor\frac{\alpha}{2}\right\rfloor}(-1)^{k}\left(\frac{t_{21}^{(k)}(z)}{(\alpha+1)^{(k+1)}}+\frac{t_{22}^{(k)}(z)}{(\alpha-2)^{(k+1)}}+\frac{t_{23}^{(k)}(z)}{(\alpha-4)^{(k+1)}}\right), \\
& h_{a}(z)=\sum_{k=0}^{\left\lfloor\frac{\alpha}{2}\right\rfloor}(-1)^{k}\left(\frac{t_{31}^{(k)}(z)}{(\alpha+1)^{(k+1)}}+\frac{t_{32}^{(k)}(z)}{(\alpha-2)^{(k+1)}}+\frac{t_{33}^{(k)}(z)}{(\alpha-4)^{(k+1)}}\right) .
\end{aligned}
$$

$f_{\alpha}(z), g_{c}(z)$ and $h_{\alpha}(z), 1 \leq \alpha \leq 4$ must be calculated by hand, as follows, taking the top value for $f_{0}$ and $g_{0}$ from $(2.30)$.

$$
\left(\begin{array}{l}
f_{0} \\
y_{0} \\
h_{0}
\end{array}\right)=\left(\begin{array}{c}
2 i \\
-2 i / \sigma \\
-2 / \sigma
\end{array}\right),
$$




$$
\begin{gathered}
\left(\begin{array}{l}
f_{1} \\
g_{1} \\
h_{1}
\end{array}\right)=\left(\begin{array}{c}
\frac{2}{3}(3 \sigma-1-2 B) \\
2 i \\
\left(\frac{2}{3 \sigma}(1-B+3 \sigma)\right.
\end{array}\right) \\
\left(\begin{array}{l}
f_{2}(z) \\
g_{2}(z) \\
h_{2}(z)
\end{array}\right)=\left(\begin{array}{c}
i \frac{3 \sigma^{2}+\sigma\left(-9 R+9 a_{h}+11-8 B\right)+3 B^{2}-2 B-1}{3} \\
i \frac{\sigma\left(-9 R+9 a_{h}+12-12 B\right)+5 B^{2}-4 B-1}{9 \sigma} \\
a_{h}
\end{array}\right)+\left(\begin{array}{c}
\frac{2 i}{9}\left(B^{2}-B+(B+2) \sigma-6 \sigma^{2}\right) \\
\frac{21}{9 \sigma}\left(B^{2}-B+(B+2) \sigma-6 \sigma^{2}\right) \\
\frac{2}{9 \sigma}\left(B^{2}-B+(B+2) \sigma-6 \sigma^{2}\right)
\end{array}\right)=
\end{gathered}
$$

where $a_{h}$ is arbitrary,

$$
\left(\begin{array}{l}
f_{3}(z) \\
g_{3}(z) \\
h_{3}(z)
\end{array}\right)=\left(\begin{array}{l}
c_{f} \\
c_{g} \\
c_{h}
\end{array}\right)+\left(\begin{array}{l}
d_{f} \\
d_{g} \\
d_{h}
\end{array}\right) z
$$

where

$$
\begin{aligned}
& c_{f}=-\left(2 i+6 i B-18 i B^{2}+10 i B^{3}+\left(-13 i-18 i a_{h}+B\left(101 i+18 i a_{h}\right)\right.\right. \\
&\left.+B-i 3 i B^{2}-15 i B^{3}+(18 i-18 i B) R\right) \sigma+\left(-169 i-90 i a_{h}+\left(62 i+36 i a_{h}\right) B+\right. \\
&\left.107 i B^{2}+(90 i+18 i B) R\right) \sigma^{2}+\left(255 i+216 i a_{h}-309 i B-216 i R\right) \\
&\left.\sigma^{3}+297 i \sigma^{4}\right) / 108 \sigma \\
& c_{g}=-\left(2 i+6 i B-18 i B^{2}+10 i B^{3}+\left(-13 i-18 i a_{h}+\left(107 i+18 i a_{h}\right)\right.\right. \\
&\left.B-85 i B^{2}-9 i B^{3}+(18 i-18 i B) R\right) \sigma+\left(-175 i-90 i a_{h}+\left(62 i+36 i a_{h}\right) B+\right. \\
&\left.113 i B^{2}+(90 i+18 i B) R\right) \sigma^{2}+\left(261 i+162 i a_{h}-279 i B-162 i R\right) \\
&\left.\sigma^{3}+171 i \sigma^{4}\right) /\left(54 \sigma^{2}\right), \\
& c_{h}=-\left(-2-6 B+18 B^{2}-10 B^{3}+\left(15+18 a_{h}+\left(-93-18 a_{h}\right) B+87 B^{2}-9 B^{3}+\right.\right. \\
&(-18+18 B) R) \sigma+\left(123+i 2 a_{h}+\left(-102-18 a_{h}\right) B-21 B^{2}+\right. \\
&(-i 2+18 B) R) \sigma^{2}+\left(-63+54\left(a_{h}+9 B-54 R\right)\right. \\
&\left.\sigma^{3}+135 \sigma^{4}\right) / 54 \sigma^{2} \\
& d_{f}=-i B+2 i B^{2}-i B^{3}+\left(2 i+2 i B-4 i B^{2}\right) \sigma+(-12 i+3 i B) \sigma^{2}+18 i \sigma^{3} \\
& y
\end{aligned}
$$




$$
\begin{gathered}
d_{g}=\frac{-2 i B+4 i B^{2}-2 i B^{3}+\left(4 i+2 i B-6 i B^{2}\right) \sigma+(-20 i+8 i B) \sigma^{2}+24 i \sigma^{3}}{9 \sigma} \text { and } \\
d_{h}=\frac{4 B-8 B^{2}+4 B^{3}+\left(-8+16 B-8 B^{2}\right) \sigma-36 B \sigma^{2}+72 \sigma^{3}}{27 \sigma} \\
\left(\begin{array}{l}
f_{A}(z) \\
g_{s}(z) \\
h_{A}(z)
\end{array}\right)=\left(\begin{array}{l}
k_{f} \\
k_{g} \\
k_{h}
\end{array}\right)+\left(\begin{array}{l}
l_{f} \\
l_{g} \\
l_{h}
\end{array}\right) z+\left(\begin{array}{l}
m_{f} \\
m_{g} \\
m_{h}
\end{array}\right) z^{2}
\end{gathered}
$$

where

$$
\begin{aligned}
k_{f}= & \left(-2 i-16 i B-12 i B^{2}+50 i B^{3}-50 i B^{4}+\left(29 i+18 i a_{h}+\left(-18+12 i+72 i a_{h}\right) B\right.\right. \\
& \left.\left(18-562 i-50 i a_{h}\right) B^{2}+540 i B^{3}-19 i B^{4}+\left(-18 i-72 i B+90 i B^{2}\right) R\right) \\
& \sigma+\left(36-36 i a_{h}+\left(1588 i+630 i a_{h}\right) B+\left(18-1376 i-270 i a_{h}\right) B^{2}-\right. \\
& \left.304 i B^{3}+i(92-324 i I I)+\left(36 i-684 i B+162 i B^{2}\right) R\right) \sigma^{2}+ \\
& \left(-72-1906 i-1386 i a_{h}-162 i a_{h}^{2}+\left(18+728 i+522 i a_{h}\right) B+1034 i B^{2}+\right. \\
& \left.\left(1386 i+324 i a_{h}-36 i B\right) R-162 i R^{2}\right) \sigma^{3}+\left(-108+1482 i+1728 i a_{h}-2256 i B-\right. \\
& \left.1728 i R) \sigma^{4}+3015 i \sigma^{5}\right) / 648 \sigma
\end{aligned}
$$$$
k_{g}=90 i-240 i B-2340 i B^{2}+5040 i B^{3}-2550 i B^{4}+\left(75 i-810 i a_{h}+\right.
$$$$
\left(-990+9808 i+5400 i a_{h}\right) B+\left(990-35522 i-4590 i a_{h}\right) B^{2}+26200 i B^{3}
$$$$
\left.-561 i B^{4}+\left(810 i-5400 i B+4590 i B^{2}\right) R\right) \sigma+\left(1980-8640 i a_{h}+\right.
$$$$
\left(144+85094 i+35910 i a_{h}\right) B+\left(846-58426 i-15930 i a_{h}\right) B^{2}-
$$$$
17222 i B^{3}+i\left(-9446-24300 k_{h}\right)+\left(8640 i-34416 i B+8766 i B^{2}\right) R
$$$$
\sigma^{2}+\left(-4248-i 3640 i-60210 i a_{h}-12150 i a_{h}{ }^{2}+\right.
$$$$
\left(846+7372 i+22950 i a_{h}\right) B+61228 i B^{2}+\left(59922 i+24300 i a_{h}+\right.
$$$$
\left.2016 i B) R-12150 i R^{2}\right) \sigma^{3}+\left(-5076+90300 i+90720 i a_{h}-\right.
$$$$
131106 i B-89856 i R) \sigma^{4}+15968 i i \sigma^{5} / 16200 \sigma^{2} \text {, }
$$

$$
l_{f}=-\left(-30 i-120 i B+180 i B^{2}+120 i B^{3}-150 i B^{4}+\left(225 i+270 i a_{h}+\right.\right.
$$


$(-45-1226 i) B+\left(45-491 i-270 i a_{h}\right) B^{2}+1600 i B^{3}-108 i B^{4}+$ $\left.\left(-270 i+270 i B^{2}\right) R\right) \sigma+\left(90+228 i i+1080 i a_{h}+\left(-18+2357 i+1080 i a_{h}\right) B+\right.$ $\left.\left(63-4228 i-5.40 i a_{h}\right) B^{2}-416 i B^{3}+\left(-1080 i-17 i 3 i B+423 i B^{2}\right) R\right) \sigma^{2}+$ $\left(-144-5495 i-3780 i a_{h}+\left(63+4741 i+135 n i a_{h}\right) B+34 i B^{2}+(3816 i-927 i B)\right.$ $\left.\sigma^{3}+\left(-3 i s-2175 i+810 i a_{h}+1032 i B-918 i R\right) \sigma^{4}+2736 i \sigma^{5}\right) / 2025 \sigma$,

$l_{g}=-\left(-90 i-360 i B+540 i B^{2}+360 i B^{3}-450 i B^{4}+\left(675 i+810 i a_{h}+\right.\right.$ $(-135-3848 i) B+\left(135-1243 i-810 i a_{h}\right) B^{2}+4850 i B^{3}-434 i B^{4}+$ $\left.\left(-810 i+810 i B^{2}\right) R\right) \sigma+\left(270+7201 i+3240 i a_{h}+\left(36+7636 i+3240 i a_{h}\right) B+\right.$ $\left(99-14144 i-1620 i a_{h}\right) B^{2}-\left(99 i B^{3}+\left(-3240 i-5229 i B+1179 i B^{2}\right) R\right) \sigma^{2}+$ $\left(-612-18535 i-11340 i a_{h}+\left(99+14843 i+4050 i a_{h}\right) B+1982 i B^{2}+\right.$ $(11268 i-2871 i B) R) \sigma^{3}+\left(-594-2325 i+2430 i a_{h}-339 i B-\right.$ $\left.2214 i R) \sigma^{4}+48 i 8 i \sigma^{5}\right) / 2025 \sigma^{2}$,

$$
\begin{aligned}
l_{h}= & -\left(60+240 B-360 B^{2}-240 B^{3}+300 B^{4}+\left(-450-540 a_{h}+(2527+135 i) B+\right.\right. \\
& \left.\left(1082-135 i+5.10 a_{h}\right) B^{2}-3625 B^{3}+466 B^{4}+\left(540-540 B^{2}\right) R\right) \sigma+ \\
& \left(-4724-270 i-2160 a_{h}+\left(-5864-36 i-2160 a_{h}\right) B+\left(10: 88-99 i+1080 a_{h}\right) B\right. \\
& \left.+207 B^{3}+\left(2160+35.16 B-8.16 B^{2}\right) R\right) \sigma^{2}+\left(12890+612 i+7560 a_{h}+\right. \\
& \left.\left(-i 957-99 i-2700 a_{h}\right) B-394.13 B^{2}+(-7632+1854 B) R\right) \sigma^{3}+ \\
& \left.\left(-3000+594 i-1620 a_{h}+1686 B+1836 R\right) \sigma^{4}+3528 \sigma^{5}\right) / 2025 \sigma^{2}
\end{aligned}
$$

$$
\begin{aligned}
m_{f}= & -\left(-17 i B+23 i B^{2}+5 i B^{3}-11 i B^{4}+(34 i+(9+34 i) B+\right. \\
& \left.(-9-101 i) B^{2}+33 i B^{3}+\left(3 i B-9 i B^{2}\right) R\right) \sigma+(-18-160 i+(-9+107 i) B \\
& \left.\left.98 i B^{2}+(-18 i-9 i B) R\right) \sigma^{2}+(54+150 i-276 i B+54 i R) \sigma^{3}+72 i \sigma^{4}\right) / 405
\end{aligned}
$$

$$
\begin{aligned}
m_{g}= & -\left(-17 i B+23 i B^{2}+5, B^{3}-11, B^{4}+(34 i+(9+34 i) B+\right. \\
& \left.(-9-101 i) B^{2}+33 i B^{3}+\left(9 i B-9 i B^{2}\right) R\right) \sigma+(-18-160 i+(-9+107 i) B \\
& \left.\left.98 i B^{2}+(-18 i-9 i B) R\right) \sigma^{2}+(54+150 i-276 i B+54 i R) \sigma^{3}+72 i \sigma^{4}\right) / 135
\end{aligned}
$$




$$
\begin{aligned}
m_{h}= & -\left(34 B-36 B^{2}-30 B^{3}+32 B^{4}+(-68+(-108+18 i) B+\right. \\
& \left.(222-18 i) B^{2}-46 B^{3}+\left(-18 B+18 B^{2}\right) R\right) \sigma+(360-36 i+(-54-18 i) B- \\
& \left.\left.306 B^{2}+(36+18 B) R\right) \sigma^{2}+(-540+108 i+432 B-108 R) \sigma^{3}+216 \sigma^{4}\right) / 405 \sigma .
\end{aligned}
$$

If $\log ^{4}(\tau)$ is required, $f g h, \alpha=0,1,2,3$, and 4 are

$$
\begin{aligned}
& f_{0}=2 i, \\
& f_{1}=\frac{i}{3}(-1-2 B+3 \sigma) \text {, } \\
& g_{0}=\frac{-2 i}{\sigma} \\
& g_{1}=2 i \text {, } \\
& h_{0}=\frac{-2}{\sigma} \\
& h_{1}=\frac{2(1-B+3 \sigma)}{3 \sigma} \text {, } \\
& f_{2}=i h_{2} \sigma-\frac{\sigma\left(R f_{0}-g_{1}-f_{1} h_{1}\right)}{2} \text {, } \\
& g_{2}=\frac{\sigma f_{1}+f(2)}{\sigma} \\
& f_{3}=\frac{-3 \sigma f(2)}{4}+\frac{i}{2} \sigma\left(f(2) g_{1}+f_{1} g(2)-B h(2)\right)-\frac{\sigma\left(R f_{1}-g_{2}-f_{2} h_{1}-f_{1} h_{2}\right)}{4}, \\
& g_{3}=\frac{-f_{2}}{2}+i\left(f_{2} g_{1}+f_{1} g_{2}-B h_{2}\right)-\frac{R f_{1}-g_{2}-f_{2} h_{1}-f_{1} h_{2}}{2}, \\
& h_{3}=\frac{i}{2} f_{2}-\frac{i}{2}\left(R f_{1}-g_{2}-f_{2} h_{1}-f_{1} h_{2}\right), \\
& \left(\begin{array}{l}
f_{4}(z) \\
g_{4}(z) \\
h_{4}(z)
\end{array}\right)=\left(\begin{array}{c}
a_{f} \\
a_{g} \\
a_{h}
\end{array}\right)+\left(\begin{array}{l}
b_{f} \\
b_{g} \\
b_{h}
\end{array}\right) z
\end{aligned}
$$

where

$$
\begin{aligned}
b_{h}= & 2 i R f_{1}+2 i B R f_{1}-4 R f_{1}^{2}+2 i f_{2}-2 i B f_{2}+4 i R j_{2}- \\
& R \sigma f_{1} g_{1}+4 f_{2} g_{1}-3 \sigma f_{2} g_{1}+4 i f_{1} f_{2} g_{1}+2 i \sigma f_{2} g_{1}^{2}- \\
& 2 i g_{2}-2 i B g_{2}+8 f_{1} g_{2}+4 i f_{1}^{2} g_{2}+4 f_{2} g_{2}+\sigma g_{1} g_{2}+
\end{aligned}
$$




$$
\begin{gathered}
2 i \sigma f_{1} g_{1} g_{2}+i R \sigma f_{1} h_{1}-2 i f_{2} h_{1}-2 i B f_{2} h_{1}+ \\
3 i \sigma f_{2} h_{1}+4 f_{1} f_{2} h_{1}+3 \sigma f_{2} g_{1} h_{1}-i \sigma g_{2} h_{1}+ \\
2 \sigma f_{1} g_{2} h_{1}-i \sigma f_{2} h_{1}{ }^{2}-4 B h_{2}-2 i f_{1} h_{2}-i i B f_{1} h_{2}+ \\
4 f_{1}{ }^{2} h_{2}-4 i f_{2} h_{2}-2 i B \sigma g_{1} h_{2}+\sigma f_{1} g_{1} h_{2}- \\
2 B \sigma h_{1} h_{2}-i \sigma f_{1} h_{1} h_{2} / 10 \\
b_{f}=\frac{-i}{2} h_{h} \sigma, \\
a_{f}=-\frac{-3 i}{2} b_{h}, \\
2 i \sigma^{2} f_{1} g_{2}-s^{2} f_{2} h_{1}+2 i B \sigma^{2} h_{2}-\sigma^{2} f_{1} h_{2} / 8 \\
a_{g}=\frac{\frac{-3 h_{2}}{2} a_{h}-h_{f}}{2 \sigma+\frac{R \sigma f_{1}}{8}+\frac{3 \sigma f_{2}}{8}-\frac{i}{4} \sigma f_{2} g_{1}-\frac{\sigma g_{2}}{8}} \\
\frac{i}{4} \sigma f_{1} g_{2}-\frac{\sigma f_{2} h_{1}}{8}+\frac{i}{4} B \sigma h_{2}-\frac{\sigma f_{1} h_{2}}{8} .
\end{gathered}
$$




\section{Bib".ugraphy}

[1] M. Adler, P. Van Moerbeke: Completely integrable systems, Euclidean Lie algebras, and curves, $\lambda d v$. Math. 38, 267 (1980).

[2] M. Adler, P. Van Moerbeke: Linearization of systems, Jacabi varieties ant representation theory, Adv. Math. 38, 318 (1980).

[3] T.C. Bountis: On nonintegrable systems with square root singularities in complex time, Phys. Lett. A 159, 1 (1991).

[4] T.C. Bountis: Nonintegrable systems with algebraic singularities in complex time, J. Phys. A 24, 3217 (1991).

[5] T.C. Bountis, M. Bier, Y. Papageorgiou: A Singularity Analysis Approach to the Solutions of Dutfing's Equation in Symmetries and Singularity Structures, ed. by M. Lakshmanan and M. Daniel, 112 (1990).

[6] T.C. Bountis, L.B. Drossos, M. Lakshmanan, S. Parthasarathy: On the Nonintegrability of a Fumily of Duffing-win der Pol Oscillators, J. Phys. A 26, 6927 (1993).

[7] T.C. Bountis, V'. Papageorgiou. M. Bier: On the Singularity Analysis of Intersecting Separatrices in Near-Integrable Dynamical Systems,Physica 24D, 292 $(198 \pi)$.

[8] T.C. Bountis, A. Ramani. B. Grammaticos, B. Dorrizo: On the Complete and Partial Integrability of Non-Hamiltonian systems, Physica 128A, 268 (1984). 
[9] T.C. Bountis. H. Segur: Logarithmic Singularities and (hnotic Behaviour in Hamillonian Systems in Mathematical Methods in Ilydrodynamics and lntegrability in Dynamical Systems ed. M. Tabor and Yvain M. Treve, 279 (1981).

[10] T.C. Bountis and H. Segur: A Singularity Analysis of Integrability and (huos in Dynamical Systems in Singularities and Dynamical Systems, ed S. Pnevmatikos (1984).

[11] T.C. Bountis, II. Segur, F. Vivaldi: Integrable Hamiltonian Systems and the Painlevé Property, Phys. Rev. A 25,1257 (1982).

[12] B. Boyce. D. Diprima: Differential Equations

[13] F. Calogero: A Solvable Nonlinear Wave Equation. Studies in Applied Mathematics 70, 1\$9 (1984).

[14] F. Bureau: Diffcrential Equations wilh Fixed Critical Points, Ann. Math. Pura Appl. (4). 64, 229 (1964).

[15] F. Bureau: Diffcrential Equations with Fixed Critical J'oints, Ann. Math. Pura Appl. (4) 66, 1 (1964).

[16] F. Bureau: Integration of some Nonlinear Systems of OIDFs, Ann. Math. Pura Appl. (4) 94. 344 (1972).

[17] F. Bureau: Sur des Système différentiels du Troisième Ordre el les équations Différentiels associées, Acad. Roy. Belg. Bull. (1. Sci. (5) 73, 3355 (1987).

[18] Y.F. Chang, J.M. Greene, M. Tabor, J. Weiss: The Analytic Structure of Dy-, namical Systems and Sclf-Similar Natural Boundaries, Physica 8D.18:3 (1983).

[19] Y.F. Chang, M. Talor, J. Wriss, (i. Corliss: On the Awalytic Structure of the Ilenon-Hlcilıs Systcm. Phy's. Lett. 185,211 (1981).

[20] Y.F. Chang, .11. Tabot, J. Weiss: Inclytic Structure of the IIenon-IIeiles IIarniltonian in Integrable and Nonintegrable Regimes, J. Math. Phys. 23, 5331 (1982). 
[21] J. Chazy: Sur les Équations Différentielles du Troisieme ordre et d'ordre supéricur dont l'intégrale générale a ses points critiques fixes, Acta Math. 34, 317 (1911).

[22] P.A. Clarkson, J.B. McCleod, P.J. Olver, A. Ramani: Integrability of KleinGordon Equations SIAM J. Math. Anal. 17, 798 (1986).

[23] C.M. Cosgrove: Painlevé Classification of all Semilinear Partial Differential Equations of the Second Order. I IIyperbolic equations in two independent variables. II. Parabolic and Higher Dimensional Equations, Studies in Applied Mathematics 89. 1 (1993).

[24] C. M. Cosgrove: All binomial-type Painleve equations of the Second Order and degree Three or Higher, Studies in Applied Mathematics 90, 119 (1993).

[25] C.M. Cosgrove, G. Scoufis: Painlevé Classification of a Class of Differential Equations of the Sccond Order and Second Degree, Studies in Applied Mathematics 88, 25, (1993).

[26] T. Dombre, U. Frisch, J.M. Gicene, M. IJenon, A. Mehr, A.M. Soward: Chaotic Streamlines in the ABC Flows. J. Fluid Mech. 167, 353 (1986).

[27] B. Dorizzi. B. Grammaticos: Explicit Integrability for Hamiltonian Śystems and the Painlevé Conjecture,J. Math. Phys. 25(3),481 (1984).

[28] B. Dorizzi. B. Grammaticos. A. Ramani, P. Winternitz: Integrable Hamiltonian Systems wilh V'rlocity-dependent Polentials, J. Math. Phys. 26, 3070 (1985).

[29] H. Flaschkia, A.C. Newell, M. Tabor: Integrability, in What is Integratility, ed. by V.E. Zakharov, 73 (1991).

[30] J.D. Fournier. G. Levine, M. Tabor: Singularity Clustering in the Duffing Oscillator. A. Phy's. A 21, 33 (1988).

[31] U. Frisch. R. Morf: Intermittency in Nonlinear Dynamics and Singularities at Complex Times. Phys. Rev. A 23. 2673 (1981). 
[32] B. Gambier: Sur les Équations Différenticlles du Second Ordre et du Premier degré dont lintégrale Giénérale est a points critiques Jixes. Acta Math. 33, I (1910).

[33] R. Garnier: Sur des Équations différentielles du Troisieme ordre dont l'intégrale Générale est Uniforme et sur une Classe d'équations nouvtlles d'ordre S'upirior dont l'intégrale Générale est Unifo -me, Ann. Sci. Ecole Norm. jup. 29. 1 (1912).

[34] B. Grammaticos, B. Dorrizo. A. Ramani: Integrability of Ilamiltonians with third and fourth degree Polynomial Potentials, J. Math. Phys. 24, 2289 (1984).

[35] B. Grammaticos, B. Dorizzi, A. Ramani: Hamiltonians with high-order Integrals and the Wenk Painlevé Concept, J. Math. Phys. 25, 3470 (1984).

[36] M.A. Hemmi: Jéries Solutıns of Nonlinear Ordinary Dilferential Equutions. Masters Thesi:s at Carelton University, (1994).

[37] J. Hietarinta, B. Dorizzi, B. Grammaticos: Complete Painlevé Analysis for Coupled Quartic Oscillators in N Dimensions, Phys. Lett. A 108, 55 (1985).

[38] E. Hille: A Note on Quadratic Sysitems, Proc. RSE(A) 72, 1 (1972).

[39] E. Hille: Differential Equations in the Complex Plane. (1976).

[40] L. Hlavaty: On the Painlevé Classification of Partial Differential Equations, J. Math. Phys 31, 605 (1990).

[41] L. Hlavaty: On the Painleve (lassification of Partial Differential Equations II. Rational Equations with One Pole. ('zechoslovak Journal of Physics 42, 7655 (1992).

[42] L. Hlavatý: On the Painlevé Classification of Partial Differential Equations III. Rational Equations with Several Poles, J. Math. Phys. 33, 888 (1092).

[43] E. Ince: Ordinar'y Differentidl Equations, (1926). 

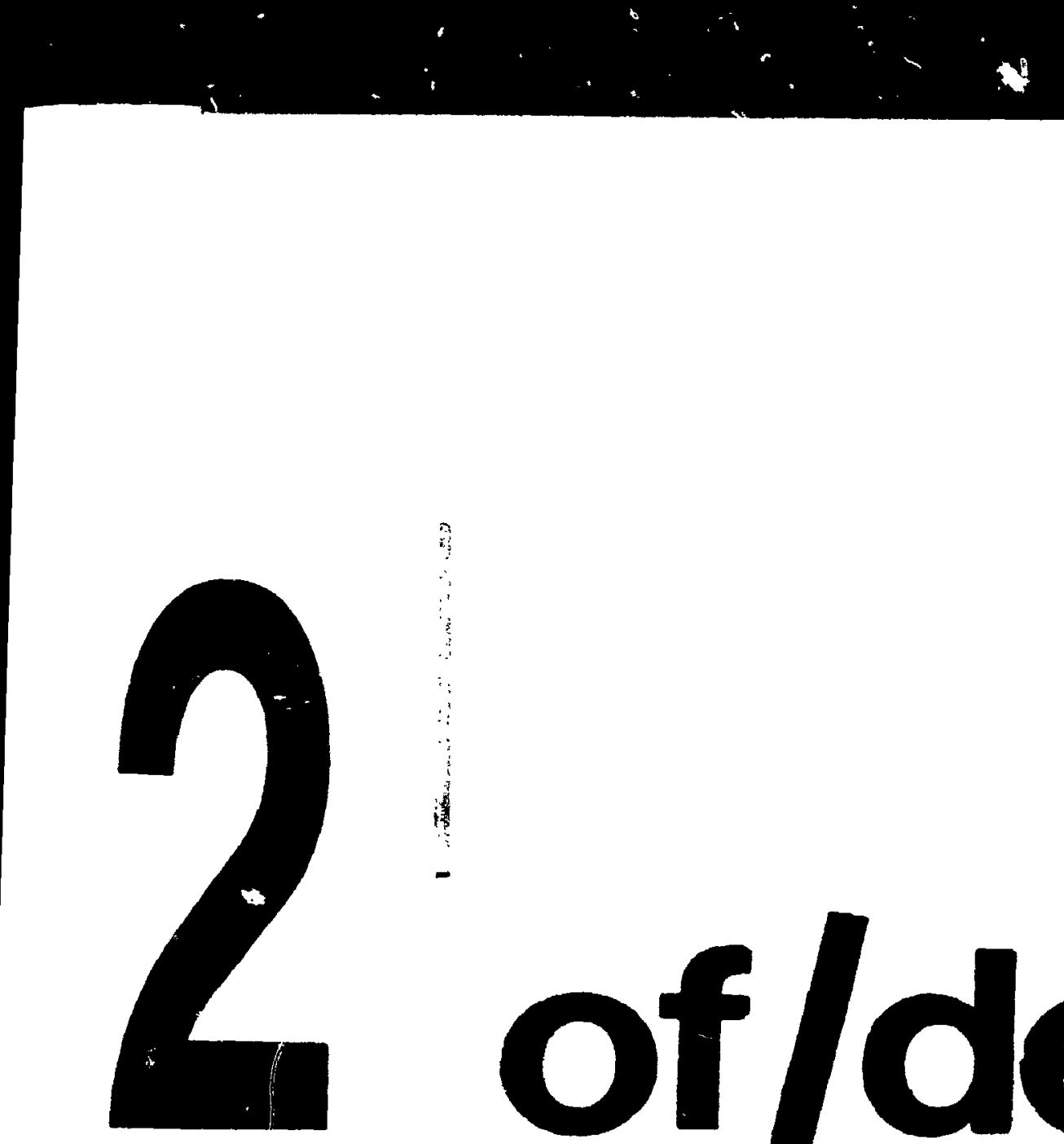

$\vdots$
$\vdots$
$\vdots$
$\vdots$
$\vdots$
$\ddots$
$\vdots$
$\vdots$
$\vdots$
-
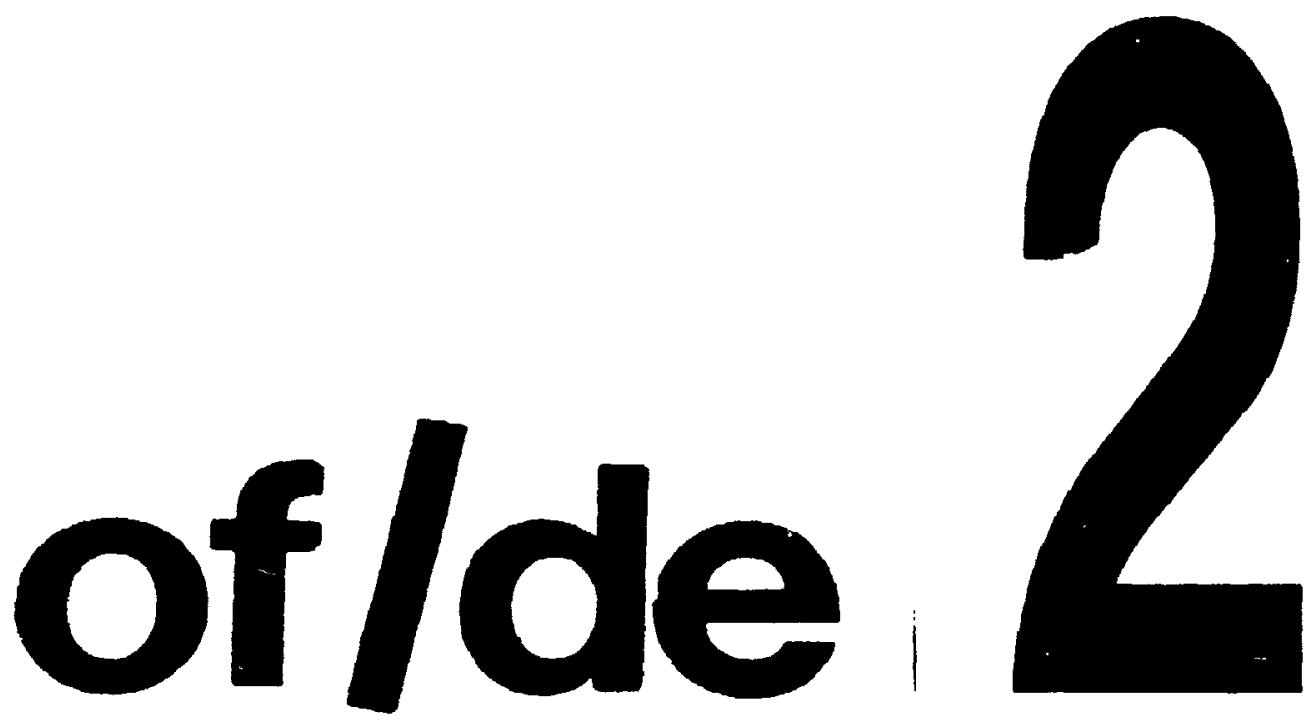

PM-1 31/2"x4" PHOTOGRAPHIC MICROCOPY TARGET NBS 1010a ANSI/ISO \#2 EOUIVALENT

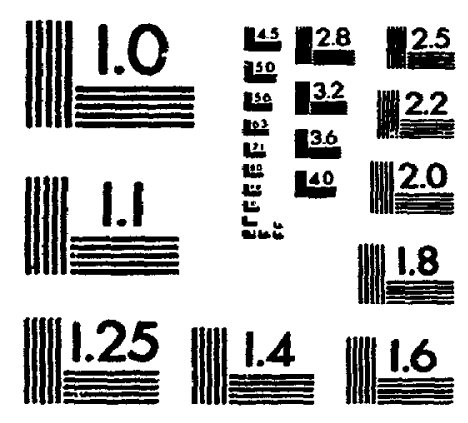

PRECISIONSW RESOLUTION TARGETS 

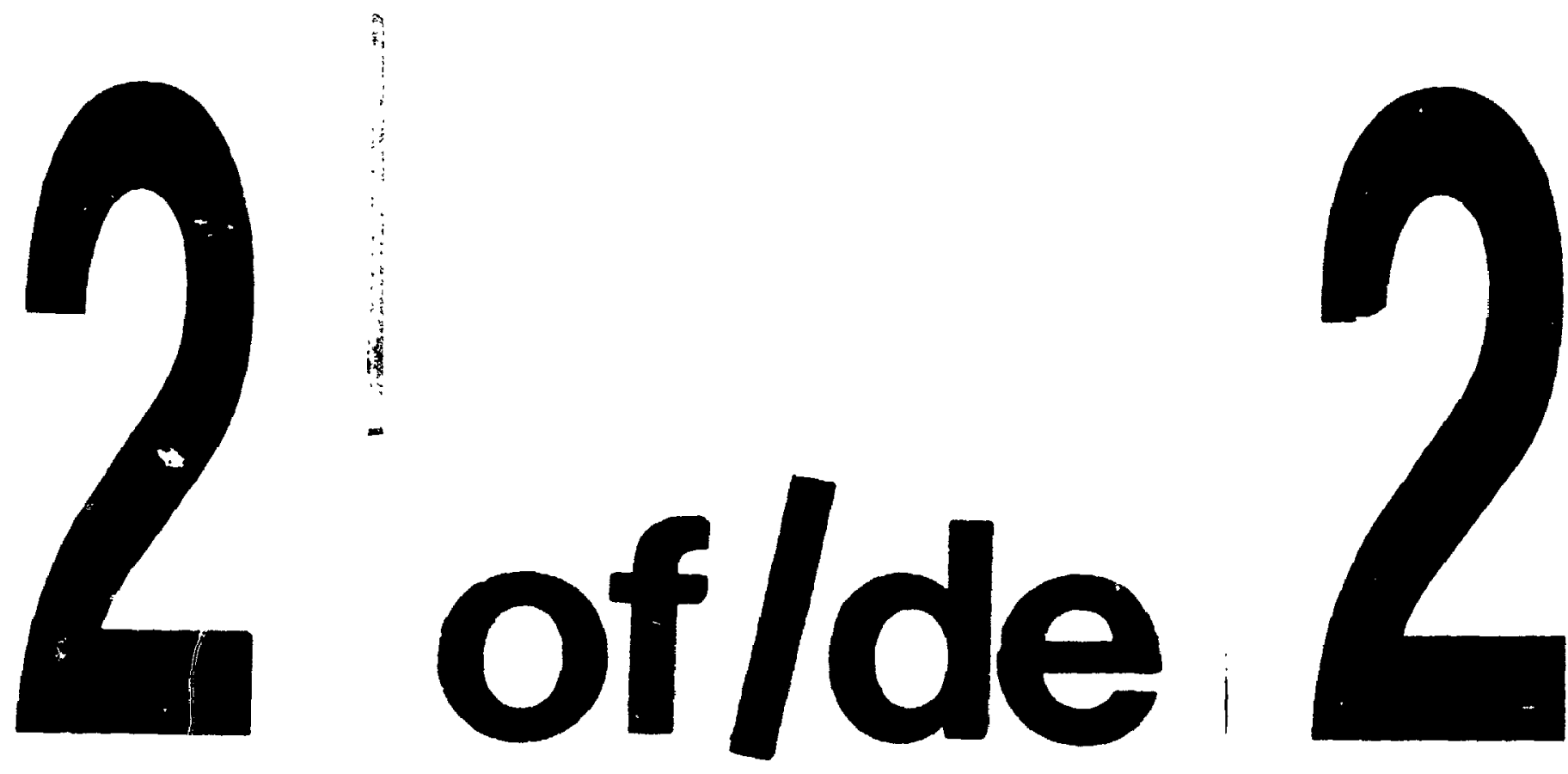

PM-1 31/2"x4" PHOTOGRAPHIC MICROCOPY TARGET NBS 1010a ANSI/ISO *2 EOUIYLENT

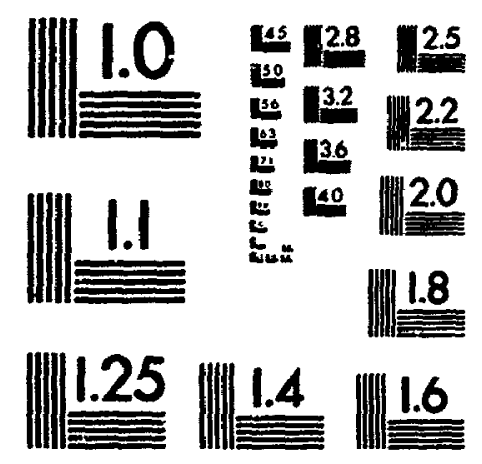

PRECISIONSW RESOLUTION TARGETS 
[44] K. Knopp: Theory and Application of Infinite Series , Blackie and Son Ltd: Glasgow (1951).

[45] S.V. Kowalevskaya: Sur lo: Problème de la Rotation d'un Corps Solide Autour d'un Point Fixe. Acta Math. 12.177 (1889).

[46] S.l. Kowalevskaya: Sur une Propriété du Système d'équations Différentielles qui Définit la Rotation d'un Coprs Solide Autour d'un Point Fixe, Acta Math. , 14, 81 (1\$89).

[47] M.D. Kruskal, P.A. Clarkson: Painlevé-Kovaleskaya and Poly-Painlevè 'ests, Studies in Applicd Math. 86, si (1992).

[48] M. Lakshmanan, R. Sahadevan: Painlevé Property of Coupled Anhurmonic Oscillators with $N$ degrees of Freedom, Phys. Lett. A 101, 189 (1984).

[49] M. Lakshmanan, R. Sahadevan: Painlevé Analysis, Lie Symmetries, and Integrability of coupled nonlinear oscillators of polynomial type, Phys. Rep. 224, 3 (1993).

[50] G. Levine, M. Tabor: Integra'ing the Non-intugable: Analytic Structure of the Lorenz System Revisited, Physica 33D 189, (1988).

[51] C.R. Menyuk, H.H. Chen. Y.C. Lee: Restricted Multiple Three-wave Interactions, Phys. Rev. A 27, 1597 (1983).

[52] S. Parthasarathy: Singularity Structures and Chaotic Dynumics of the Farametrically Driven Duffing Oscillator in Symmetries and Singularity Structures, ed. by M. Lakshmanan and M. Daniel, 104 (1990).

[53] S. Parthasarathy, M. Lakshmanan: On the Analytic Structure of the Driven Pendulum. J. Phys. A 23 L1223 (1990).

[54] S. Parthasarathy, M. Lakslımanan: Analytic Structure of the Damped Driven Morse Oscillator. Phys. Lett. A 157, 365 (1991). 
[55] S. Puri: Painlevé Property in Hamillonian and Non-Hamiltonian Systems in Symmetries and Singularity Sturctures, ed. by by M. Lakshmanan and M. Daniel, 92 (1990).

[56] A. Ramani, B. Dorizzi, B. Grammaticos, T.C. Bountis: Integrability and the Painlevé Property for Low-dimensional Systems, J. Math. Phys. 25, 878 (1984).

[57] A. Ramani, B. Grammaticos, T.C. Bountis: The Fainlevé Property and Singularity Analysis of Integrable and Non-integrab!e Systems, i'hys.Rep.180.159 (1989).

[58] A.F. Ranada, A. Ramani, B. Dorizzi, B. Grammaticos: The Weak Painlevé property as a Criterion for the Integrability of Dynamical Systems, J. Math. Phys. 26 708, (1985).

[59] R. Sahadevan and M. Lakshmanan: Invariance and Integrability: Hénon-Heiles and two coupled quartic anharmonic oscillator systems, J. Phys.A 19, 949 (1986).

[60] T. Sen, M. Tabor: Lie Symmetries of the Lorenz Model, Physica 44D,313 (1990).

[61] C. Sparrow: The Lorenz Equations: Bifurcations, Chaos and Strange Attractors (198:).

[62] M. Tabor: Modern Dynumics and Classical Analysis, Nature 310,277 (1984).

[63] M. Tabor: Chaos and Integrability in Nonlinear Dynamics (1989).

[64] M. Tabor: Analytic Structure of Dynamical Systems, Pramana - J. Phys. 2, 315 (1989).

[65] M. Tabor. J. Weiss: Analytic Structure of the Lorenz System, Phys. Rev. A 24, 2157 (198i).

[66] J. Weiss, M. Tabor, G. Carnevale: The Painlevé Property for Partial Differential Equations. J. Math. Phys. 24(3),522 (1983). 
[67] Y. Ueda: Explosion of Strange Altractors Exhibited by Duffing's Equation, Ann. N.Y Acad. Sci. 357 (19s0).

[68] S.L. Ziglin: Splitting of Separatrices. Branching of Solutions and Nonexistence of an Integral in the Dynumics of a Solid Body, Trans. Moscow Math. Soc.. 283 (19S2). 

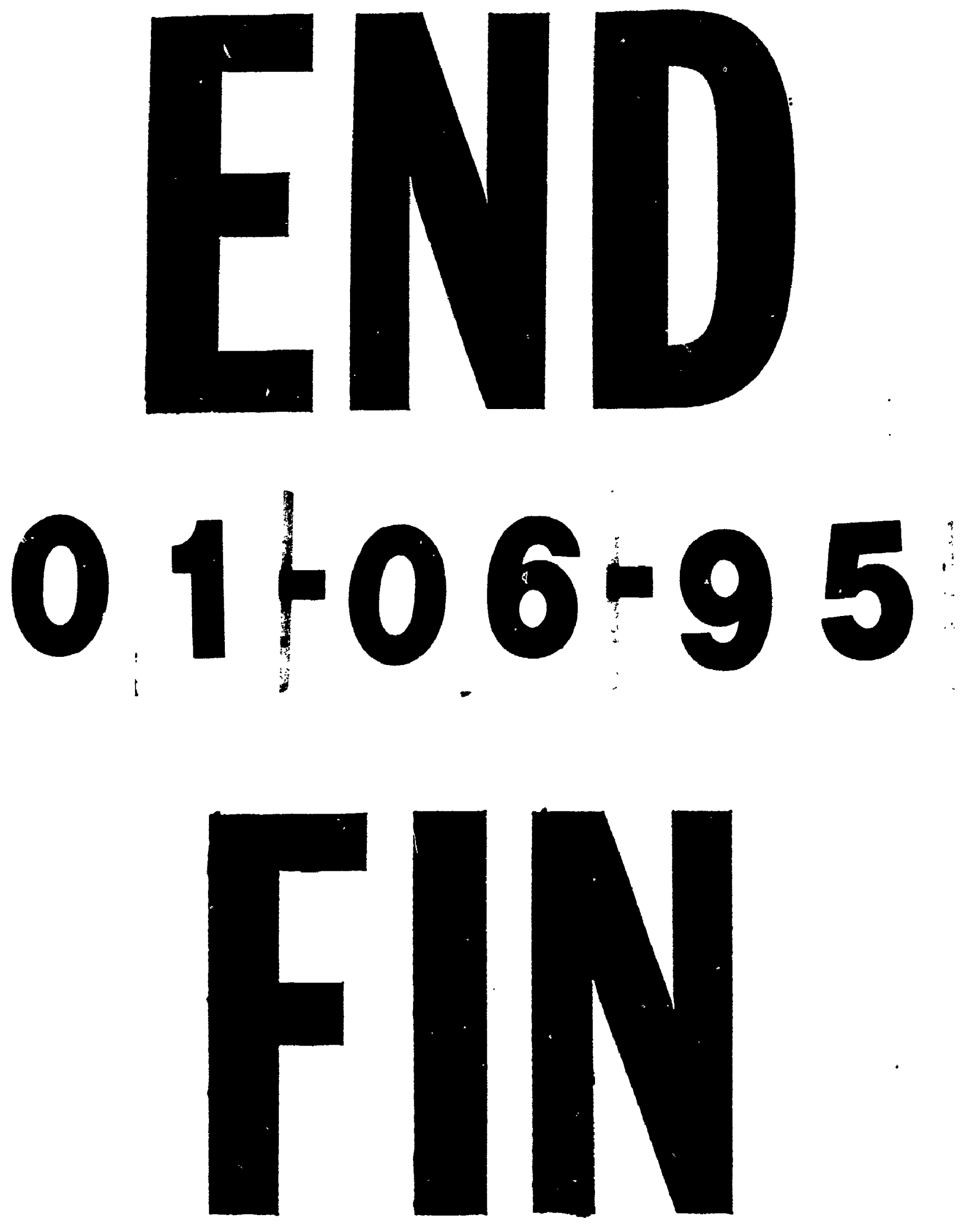